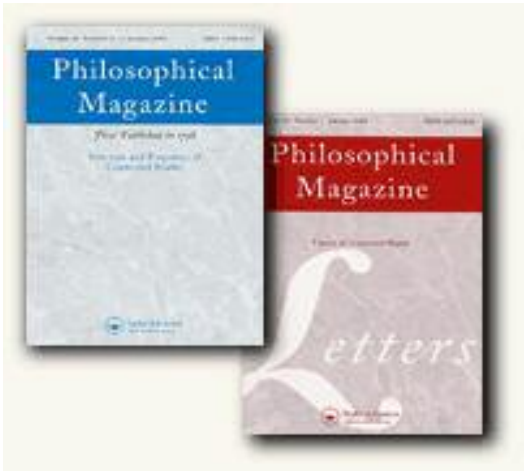

\title{
Computational homogenization of material layers with micromorphic mesostructure
}

\begin{tabular}{|c|c|}
\hline Journal: & Philosophical Magazine \& Philosophical Magazine Letters \\
\hline Manuscript ID: & TPHM-08-Jan-0001.R1 \\
\hline Journal Selection: & Philosophical Magazine \\
\hline $\begin{array}{r}\text { Date Submitted by the } \\
\text { Author: }\end{array}$ & 31-Jul-2008 \\
\hline Complete List of Authors: & $\begin{array}{l}\text { Hirschberger, Claudia; University of Kaiserslautern, Department of } \\
\text { Mechanical and Process Engineering } \\
\text { Sukumar, Natarajan; University of California, Davis, Department of } \\
\text { Civil and Environmental Engineering } \\
\text { Steinmann, Paul; University of Erlangen-Nuremberg, Department of } \\
\text { Mechanical Engineering }\end{array}$ \\
\hline Keywords: & $\begin{array}{l}\text { computational mechanics, finite-element modelling, interfaces, } \\
\text { multiscale modelling }\end{array}$ \\
\hline Keywords (user supplied): & homogenization, micromorphic continua \\
\hline \multicolumn{2}{|c|}{$\begin{array}{l}\text { Note: The following files were submitted by the author for peer review, but cannot be converted } \\
\text { to PDF. You must view these files (e.g. movies) online. }\end{array}$} \\
\hline 2007_cbh_philmag_iutam.t & \\
\hline
\end{tabular}

\section{ScholarONE" \\ Manuscript Central}




\title{
Computational homogenization of material layers with micromorphic mesostructure
}

\author{
C. B. HIRSCHBERGER ${ }^{* \dagger}$, N. SUKUMAR ${ }^{\ddagger}$, P. STEINMANN $\S$ \\ ${ }^{\dagger}$ Department of Mechanical and Process Engineering, University of Kaiserslautern, \\ P.O. Box 3049, 67653 Kaiserslautern, Germany \\ ${ }^{\ddagger}$ Department of Civil and Environmental Engineering, University of California, Davis, \\ One Shields Avenue, Davis, CA 95616, U.S.A. \\ $\S$ Department of Mechanical Engineering, University of Erlangen-Nuremberg, \\ Egerlandstraße 5, 91058 Erlangen, Germany
}

(July 30, 2008)

\begin{abstract}
In this paper, a multiscale approach to capture the behaviour of material layers that possess a micromorphic mesostructure is presented. To this end, we seek to obtain a macroscopic traction-separation law based on the underlying meso and microstructure. At the macro level, a cohesive interface description is used, whereas the underlying mesostructure is resolved as a micromorphic representative volume element. The micromorphic continuum theory is particularly well-suited to account for higher-order and size-dependent effects in the material layer. On considering the height of the material layer, quantities at different scales are related through averaging theorems and the Hill condition. An admissible scale-transition is guaranteed via the adoption of customized boundary conditions, which account for the deformation modes in the interface. On the basis of this theoretical framework, computational homogenization is embedded within a finite-element approach, and the capabilities of the model are demonstrated through numerical examples.
\end{abstract}

\section{Introduction}

Thin material layers that connect two bulk materials are found in many engineering materials applications. For instance, laminated composite structures, building materials such as masonry, and geomaterials are a few notable examples, one of which is illustrated in Figure 1. Mechanically such a material layer is usually treated as a cohesive interface within a continuum. For the constitutive relation, cohesive laws have been proposed that relate the traction and the deformation jump. However, most approaches assume that the tangential and normal responses of the interface are uncoupled (for example, the cohesive law of $\mathrm{Xu}$ and Needleman [1]), and therefore might not properly account for mixed-mode loading conditions. Remedies for the constitutive treatment of this more general case have been proposed in Reference [2].

These purely phenomenological approaches reach their limit of applicability when the material layer possesses a multiscale meso-/microstructure. On the one hand, mesostructural heterogeneities in the geometry (voids or inclusions) or the stiffness govern the material behaviour, which is not captured easily by macroscopic constitutive laws. Furthermore, intrinsic microstructural features can also be found, for instance in geomaterials such as rock or in metallic alloys that are composed of an assembly of grains. If the typical microstructural length (e.g., the grain size in a granular material) is relatively large compared to the size of the material layer, size effects become pronounced, see References $[3,4]$.

In this paper, we employ the concept of homogenization to obtain the macroscopic response of the material layer based on its underlying meso- and microstructure. Based on the assumption that the material layer is thin compared to the total specimen, it is treated as a cohesive interface embedded within a continuum at the macro scale. Therefore from a macroscopic perspective we see a discontinuity only, which on closer inspection, i.e. on the meso level appears continuous. The cohesive interface provides a sound framework that permits the used of a straightforward vectorial representation of the material

*Corresponding author. E-mail: c.b.hirschberger@tue.nl. New address: Department of Mechanical Engineering, Eindhoven University of Technology, P.O. Box 513, 5600 MB Eindhoven, The Netherlands.

Philosophical Magazine

ISSN 1478-6435 print/ISSN 1478-6443 online (c) 200x Taylor \& Francis

http://www.tandf.co.uk/journals

DOI: 10.1080/1478643YYxxxxxxxx 
layer deformation and the tractions transmitted across the layer. For the underlying mesostructure, a representative volume element (RVE) is used, which implies a statistically representative mesostructure, see e. g. the monograph [5]. Both scales are linked via homogenization, i. e., the averages of the characteristic quantities over the representative volume element are linked to the corresponding macro quantities under fulfilment of equivalence conditions coined as the Hill condition, which goes back to the contributions of Hill $[6,7]$. The finite height of the mesostructure is directly given by the thickness of the material layer, whereas the particular deformation modes that can occur within the interface are reflected by the choice of appropriate boundary conditions on the representative volume element. Besides structural size effects that are induced by heterogeneities and also treated in Reference [8], additionally a size effect, which occurs when considering a relatively large intrinsic microstructure, is captured by employing a micromorphic continuum on the level of the representative volume element. This micromorphic continuum was first introduced by Eringen [9-11] and has recently been elaborated by Kirchner and Steinmann as well as Hirschberger et al. [12-15]. It is characterized by so-called microcontinua that are endowed to each continuum point. The deformation of these kinematically independent microcontinua is considered separately. Within the constitutive assumption, an internal-length parameter is introduced that reflects the size dependence, and furthermore, a coupling between meso and micro deformation is provided. The proposed homogenization framework is implemented within a finite element method along the lines of previous approaches [16-20] and subsequent refinements that account for the influence of higher gradients [21,22]. In the multiscale finite-element solution scheme, we utilize cohesive interface elements to represent the material layer at the macro level and a micromorphic finite-element boundary value problem on the representative volume element to account for the mesostructure of the interface. Similar approaches can be found in the literature, e.g. with a micropolar continuum at the macro level [23] or in the gradient-enhanced homogenization of thin sheet structures [24]. The use of cohesive interface elements dates back to the 1980s [25] and has been employed in the modelling of decohesion in Reference [26-28], plastic localization in References [29-33], and has been combined with a discontinuous Galerkin approach towards the efficient numerical treatment of discontinuities in Reference [34].

In two-dimensional continua, which is the focus in this paper, the interface elements are one-dimensional. On the macro level, the contribution of each interface element to the global macro stiffness and the macro residual is obtained by a numerical integration that involves vectorial traction and separation quantities rather than the complete stress and strain tensor. The constitutive relation between the traction and the interface separation is numerically evaluated at each integration point of the interface element via the finite-element solution of the mesostructural boundary value problem. As indicated before, we model this meso boundary value problem such that it accounts for a representative geometry and the composition of the underlying microstructure. Since the micromorphic finite elements within the RVE incorporate both standard and additional micromorphic degrees of freedom, a nonlinear meso-micro coupled system of equation is solved. From the computational homogenization procedure, the macro traction vector and the tangent operator are obtained from the RVE and embedded into the iterative solution of the nonlinear 

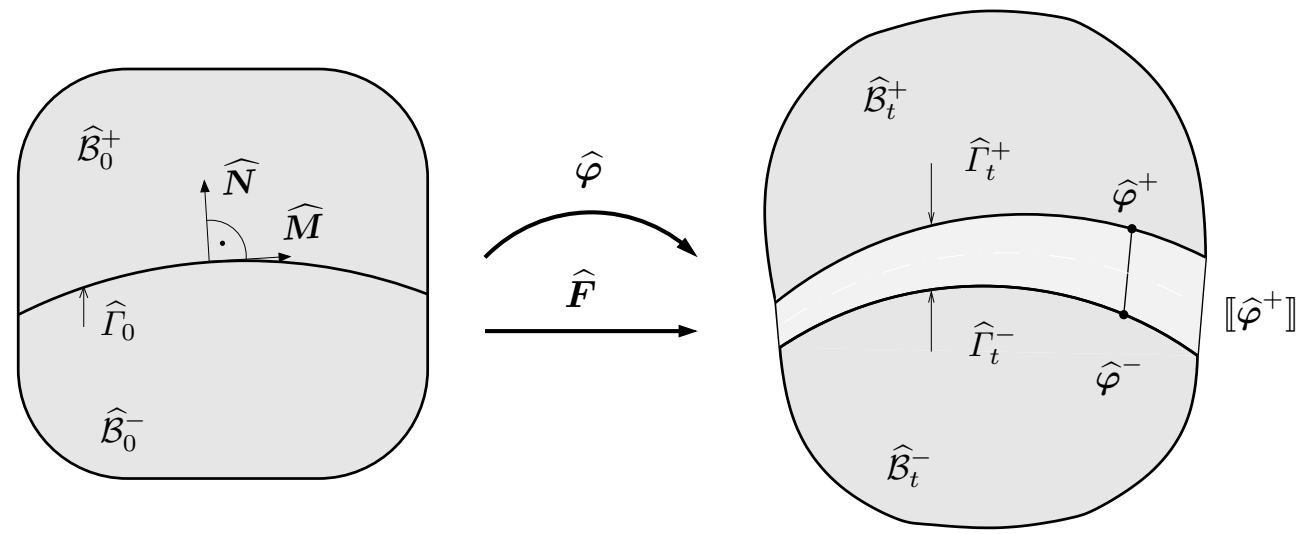

Figure 2. Interface geometry and deformation maps from the material configuration $\widehat{\Gamma}_{0}$ to the spatial configuration, $\widehat{\Gamma}_{t}$.

macro boundary value problem.

Outline. The remainder of this paper is organized as follows. In Section 2, we recall the continuum mechanics framework for the macro level, before we introduce the micromorphic representative volume element in Section 3. In Section 4, the homogenization framework to consistently link these scales is presented. The numerical approach of computational homogenization is outlined in Section 5, which is followed by benchmark numerical examples in Section 6 that display the effect of the micromorphic mesostructure on the macroscopic response. The paper is concluded in Section 7 with a summary and an outlook on topics for future research that stem from this contribution.

\section{Material layer as an interface at the macro level}

At the macro level, we consider a body that consists of a bulk material, which is separated into two parts by a very thin layer possessing decisively different properties. We treat this layer as an interface, $\widehat{\Gamma}_{0}$, and define a material normal vector $\widehat{N}$ onto the plane of the interface, which identifies the positive and the negative part of $\widehat{\mathcal{B}}_{0}$, i. e. $\widehat{\mathcal{B}}_{0}^{+}$and $\widehat{\mathcal{B}}_{0}^{+}$:

$$
\widehat{\boldsymbol{N}}(\widehat{\boldsymbol{X}})=-\widehat{\boldsymbol{N}}^{+}(\widehat{\boldsymbol{X}})=+\widehat{\boldsymbol{N}}^{-}(\widehat{\boldsymbol{X}}) \quad \forall \widehat{\boldsymbol{X}} \in \widehat{\Gamma}_{0}
$$

as illustrated in Figure 2. Note that the plane of the interface reduces to a line for the two-dimensional case. For the sake of completeness, we now briefly recall the governing equation for the bulk, and introduce the problem setting for the macro perspective on the material layer based on Reference [8].

\subsection{Deformation}

The deformation of the bulk is described by the canonical deformation mapping from the material configuration $\widehat{\mathcal{B}}_{0}$ to the spatial configuration $\widehat{\mathcal{B}}_{t}$, namely by the deformation map $\widehat{\boldsymbol{x}}=\widehat{\boldsymbol{\varphi}}(\widehat{\boldsymbol{X}})$ and its gradient $\widehat{\boldsymbol{F}}:=\nabla_{\boldsymbol{X}} \widehat{\boldsymbol{\varphi}}$ for all material points $\widehat{\boldsymbol{X}} \in \widehat{\mathcal{B}}_{0}^{+} \cup \widehat{\mathcal{B}}_{0}^{-} \backslash \widehat{\Gamma}_{0}$, compare Figure 2. A separate consideration for the interface is introduced based on the assumption that its thickness is very small compared to the bulk dimensions and the extension of the material layer. The relevant deformation quantity is the separation of the opposite edges $\widehat{\Gamma}_{t}^{+}$and $\widehat{\Gamma}_{t}^{-}$in the spatial configuration,

$$
\llbracket \widehat{\varphi} \rrbracket(\widehat{\boldsymbol{X}}):=\widehat{\varphi}^{+}(\widehat{\boldsymbol{X}})-\widehat{\boldsymbol{\varphi}}^{-}(\widehat{\boldsymbol{X}}) \quad \forall \widehat{X} \in \widehat{\Gamma}_{0}
$$




\section{$2.2 \quad$ Balance relations}

The body $\widehat{\mathcal{B}}_{0}$ is in equilibrium when the balance of momentum and the corresponding Neumann boundary condition and Dirichlet boundary condition are fulfilled:

$$
\operatorname{Div} \widehat{\boldsymbol{P}}=\mathbf{0} \quad \text { in } \widehat{\mathcal{B}}_{0} \backslash \widehat{\Gamma}_{0} \quad \widehat{\boldsymbol{P}} \cdot \widehat{\boldsymbol{N}}^{\text {ext }}=: \widehat{\boldsymbol{t}}_{0}^{\mathrm{ext}} \text { on } \partial \widehat{\mathcal{B}}_{0}^{t} \quad \widehat{\boldsymbol{u}}=\widehat{\boldsymbol{u}}^{\text {ext }} \quad \text { on } \partial \widehat{\mathcal{B}}_{0}^{u}
$$

In the above equation, the Piola stress $\widehat{\boldsymbol{P}}$ is energetically conjugate to the deformation gradient $\widehat{\boldsymbol{F}}, \widehat{\boldsymbol{t}}_{0}$ ext denotes the prescribed traction vector, and $\widehat{\boldsymbol{u}}^{\text {ext }}$ the prescribed displacement vector. Body forces are omitted for the sake of simplicity.

We assume that, across the interface, cohesive tractions are transmitted. Thus the additional equilibrium conditions must hold for the opposite sides of the cohesive interface, $\widehat{\Gamma}_{0}^{+}$and $\widehat{\Gamma}_{0}^{-}$respectively

$$
\llbracket \widehat{\boldsymbol{P}} \rrbracket \cdot \widehat{\boldsymbol{N}}=\mathbf{0}, \quad\{\widehat{\boldsymbol{P}}\} \cdot \widehat{\boldsymbol{N}}=\widehat{\boldsymbol{t}}_{0} \quad \text { on } \widehat{\Gamma}_{0},
$$

where $\llbracket \widehat{\boldsymbol{P}} \rrbracket$ denotes the jump and $\{\widehat{\boldsymbol{P}}\}$ the average of the Piola stress across the interface. Furthermore $\widehat{\boldsymbol{t}_{0}}$ represents the spatial traction vector with respect to material reference, which is transmitted across the interface. The weak formulation of the balance of momentum comprises both the virtual work terms of the bulk and the interface:

$$
\int_{\widehat{\mathcal{B}}_{0}} \widehat{\boldsymbol{P}}: \delta \widehat{\boldsymbol{F}} \mathrm{d} V+\int_{\widehat{\Gamma}_{0}} \widehat{\boldsymbol{t}}_{0} \cdot \llbracket \delta \widehat{\boldsymbol{\varphi}} \rrbracket \mathrm{d} A=\int_{\partial \widehat{\mathcal{B}}_{0}} \widehat{\boldsymbol{t}}_{0}^{\mathrm{ext}} \cdot \delta \widehat{\boldsymbol{\varphi}} \mathrm{d} A
$$

Herein the contribution of the interface to the virtual work density is $\delta \widehat{\mathcal{W}}_{0}^{\text {int }}=\widehat{\boldsymbol{t}}_{0} \cdot \llbracket \delta \widehat{\varphi} \rrbracket$, wherein the superscript (o) ${ }^{\text {int }}$ refers to 'interface'.

\subsection{Constitutive relation}

A constitutive relation is needed in order to relate the stress, respectively the traction, and the deformation measures in Equation (5) to each other. For the surrounding bulk, for the sake of simplicity we pose an a priori constitutive assumption based on a hyperelastic framework. In the cohesive interface, standard approaches would employ a cohesive traction-separation law. Therein the traction is expressed as a function of the separation, $\widehat{\boldsymbol{t}_{0}}(\llbracket \widehat{\boldsymbol{\varphi}} \rrbracket)$. Further dependencies will occur in the more general case of an inelastic material, e.g., accounting for the deformation history and the deformation state, but will not be considered here. However, these a priori choices are empirical and phenomenological, which motivates us to rather seek a relation between traction and separation based on the properties of the underlying mesostructure in the interfacial material.

Since a Newton-Raphson procedure is used in the numerical solution, we are particularly interested in the macro tangent operator $\widehat{\boldsymbol{A}}$ that appears in the incremental formulation:

$$
\delta \widehat{\boldsymbol{t}_{0}}=\widehat{\boldsymbol{A}} \cdot \llbracket \delta \widehat{\varphi} \rrbracket, \quad \widehat{\boldsymbol{A}}:=\mathrm{D}_{\llbracket \widehat{\varphi} \rrbracket} \widehat{\boldsymbol{t}_{0}} .
$$

The aim of this study is to present an appropriate multiscale framework to obtain this relation. Thereby we currently restrict our consideration to a purely hyperelastic material in the underlying micromorphic mesostructure, from which a hyperelastic response follows at the macro level. The mesostructure will be treated using the concept of representative volume elements, which we examine in the following section. 


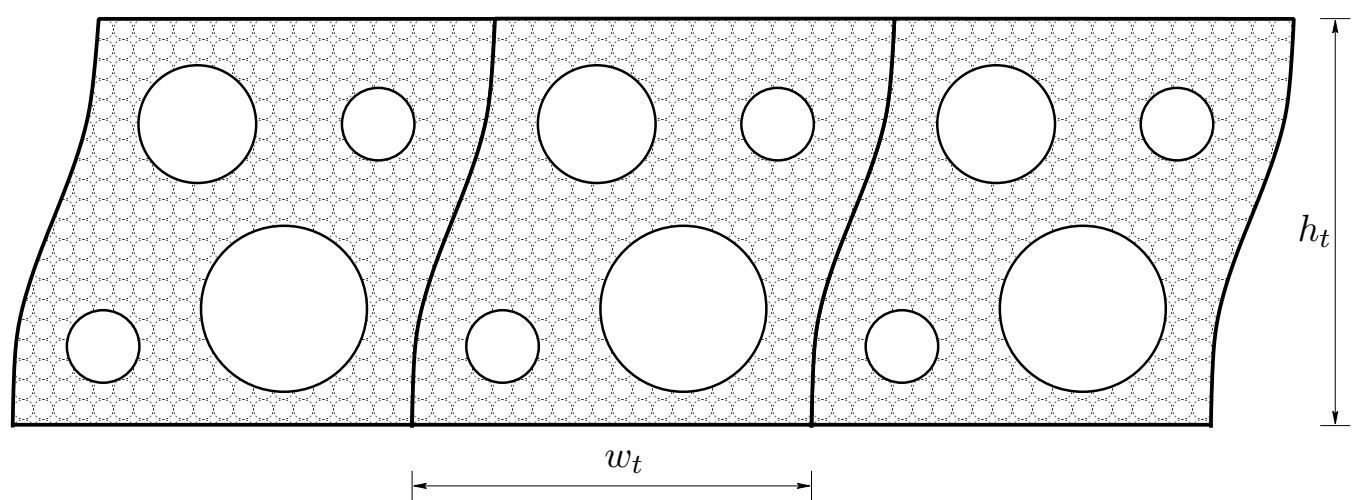

Figure 3. Material layer with periodic mesostructure and inherent microstructure: choice of the RVE height $h_{t}$ and width $w_{t}$.

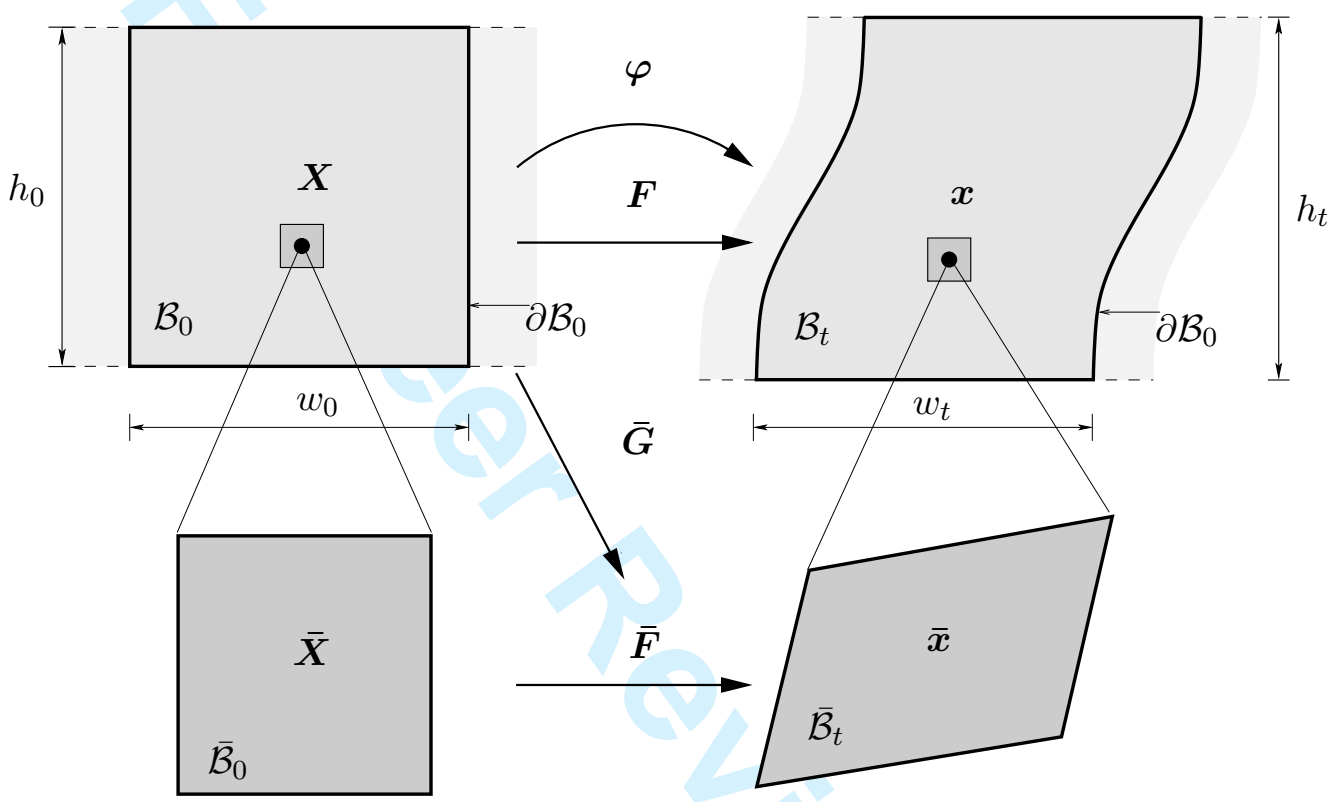

Figure 4. Micromorphic representative volume element: deformation maps from $\mathcal{B}_{0}$ to $\mathcal{B}_{t}$.

\section{Micromorphic representative volume element at the meso level}

We use the concept of representative volume element (RVE) to model the mesostructure underlying to a material segment of the interface. This RVE is required to be statistically representative for the corresponding infinitesimal material element at the macro level. Since we only consider the material layer, the finite height is adopted as a finite height of the RVE, as illustrated in Figure 3. After a few remarks on the RVE's geometry, we briefly recall the governing equations of the micromorphic RVE which will be related to the interface framework later on.

\subsection{Geometry}

The RVE is aligned to the interface in such a way that the geometric compatibility between the interface and the RVE is achieved. The initial height $h_{0}$ of the RVE coincides with the initial height of the material layer and the RVE is aligned with the interface plane. The initial width $w_{0}$ must be chosen sufficiently large to make the unit element representative, yet small compared to the in plane dimension of the interface. With this choice, the initial volume of the RVE computes as $V_{0}=h_{0} w_{0}$. Accordingly, the spatial height, width and volume are denoted as $h_{t}, w_{t}$, and $V_{t}$, respectively. 


\subsection{Deformation}

The micromorphic deformation within the RVE is described within the framework of finite-deformation kinematics in the spirit of Eringen [9-11]. As illustrated in Figure 4, at each mesocontinuum point, a socalled microcontinuum is attached, which deforms homogeneously and kinematically independently from the mesocontinuum.

The kinematic description of both the mesocontinuum points and the corresponding microcontinua is captured by considering a meso deformation map $\varphi$ and its gradient $\boldsymbol{F}$ as in a classical Boltzmann continuum plus additional quantities for the micro kinematics. In particular the micro deformation map $\overline{\boldsymbol{F}}$ being a second-order tensor represents an additional primary kinematic variable. Moreover its gradient with respect to meso placement $\bar{G}$ is introduced which consequently is a tensor of third order.

$$
\boldsymbol{x}=\boldsymbol{\varphi}(\boldsymbol{X}), \quad \boldsymbol{F}(\boldsymbol{X}):=\nabla_{\boldsymbol{X}} \varphi(\boldsymbol{X}), \quad \overline{\boldsymbol{x}}=\overline{\boldsymbol{F}}(\boldsymbol{X}) \cdot \overline{\boldsymbol{X}}, \quad \overline{\boldsymbol{G}}(\boldsymbol{X}):=\nabla_{\boldsymbol{X}} \overline{\boldsymbol{F}}(\boldsymbol{X}) .
$$

Further details can be found in References [12-15].

\subsection{Balance relations}

Within the micromorphic RVE, the local form of the balance of momentum reads:

$$
\operatorname{Div} \boldsymbol{P}=\mathbf{0} \quad \text { in } \mathcal{B}_{0}, \quad \operatorname{Div} \overline{\boldsymbol{Q}}-\overline{\boldsymbol{P}}=\mathbf{0} \quad \text { in } \mathcal{B}_{0},
$$

in terms of the meso stress $\boldsymbol{P}$, the micro stress $\overline{\boldsymbol{P}}$, and the double stress $\overline{\boldsymbol{Q}}$ of Piola type, omitting body forces. Note that the meso and the micro stress are tensors of second order, whereas the the double stress $\bar{Q}$ is a third-order tensor.

The Neumann and the Dirichlet boundary conditions are given by the relations

$$
\boldsymbol{P} \cdot \boldsymbol{N} \equiv \boldsymbol{t}_{0}^{\text {pre }} \quad \text { on } \partial \mathcal{B}_{0}^{P}, \quad \overline{\boldsymbol{Q}} \cdot \boldsymbol{N} \equiv \overline{\boldsymbol{t}}_{0}^{\text {pre }} \text { on } \partial \mathcal{B}_{0}^{\bar{Q}}, \quad \boldsymbol{\varphi} \equiv \boldsymbol{\varphi}^{\text {pre }} \text { on } \partial \mathcal{B}_{0}^{\varphi}, \quad \overline{\boldsymbol{F}} \equiv \overline{\boldsymbol{F}}^{\text {pre }} \text { on } \partial \mathcal{B}_{0}^{\bar{F}}
$$

for the traction vector $\boldsymbol{t}_{0}$ and the double traction tensor $\overline{\boldsymbol{t}}_{0}$, as well as for the meso and micro deformation map. Note that all boundary tractions and deformations act on the boundary of the meso level, $\partial \mathcal{B}_{0}$. Thereby within each scale, the boundaries follow the usual disjointness requirement, $\partial \mathcal{B}_{0}^{P} \cap \partial \mathcal{B}_{0}^{\varphi}=\emptyset$ and $\partial \mathcal{B}_{0}^{\bar{Q}} \cap \partial \mathcal{B}_{0}^{\bar{F}}=\emptyset$, whereas the boundary regions for the meso and the micro quantities are mutually independent.

\subsection{Constitutive behaviour}

A hyperelastic framework is assumed, for which the stored energy density acts as a potential. Thus the Piola-type stress measures are derived as the energetically conjugate measures to the above-introduced deformation measures as:

$$
\boldsymbol{P}:=\mathrm{D}_{\boldsymbol{F}} \mathcal{W}_{0}, \quad \overline{\boldsymbol{P}}:=\mathrm{D}_{\overline{\boldsymbol{F}}} \mathcal{W}_{0}, \quad \overline{\boldsymbol{Q}}:=\mathrm{D}_{\overline{\mathbf{G}}} \mathcal{W}_{0}
$$

These are evaluated for a particular constitutive assumption. Here the micromorphic hyperelastic formulation for the stored energy density proposed by Hirschberger et al. [14] is adopted:

$$
\mathcal{W}_{0}=\frac{1}{2} \lambda \ln ^{2} J+\frac{1}{2} \mu\left[\boldsymbol{F}: \boldsymbol{F}-n^{\operatorname{dim}}-2 \ln J\right]+\frac{1}{2} \mu l^{2} \overline{\boldsymbol{G}}: \cdot \overline{\boldsymbol{G}}+\frac{1}{2} p[\overline{\boldsymbol{F}}-\boldsymbol{F}]:[\overline{\boldsymbol{F}}-\boldsymbol{F}] .
$$

where the Jacobian determinant $J:=\operatorname{det} \boldsymbol{F}$, and $\mu$ and $\lambda$ are the Lamé material parameters. The internallength parameter $l$ accounts for the size of the microstructure and the parameter $p$ controls the scale transition between meso and micro scale. 


\subsection{Boundary value problem}

The boundary value problem on the RVE level needs to be solved according to boundary conditions imposed by the interface at the macro level. The particular relations between the RVE and the interface will be consistently addressed in the context of the scale transition in the following section.

\section{Meso-macro transition}

Between the cohesive interface at the macro level and the micromorphic RVE on the meso level, a scale transition is achieved via a homogenization. The governing quantities in the interface, i.e. the traction, the deformation jump and the virtual work, are related to the averages of the corresponding quantities within the RVE. The macro deformation or traction are applied as boundary conditions on the RVE, which must fulfil the Hill condition. We present different consistent options for these boundary conditions, before we introduce a hybrid set of boundary conditions that accounts particularly well for the geometry and deformation modes imposed by the material layer.

\subsection{Homogenization}

Since the continuum embedding the interface at the macro level is considered as a standard continuum, and within the RVE we consider a continuum of higher order, we relate the micromorphic meso quantities to the corresponding measures in the interface. The micromorphic micro quantities are implicitly accounted for by the micromorphic virtual work and the coupling in the stored energy-density formulation.

4.1.1 Deformation. The average deformation in the RVE needs to be related to a macroscopic deformation measure in the interface. To this end, we avail ourselves of a tensorial quantity as it is commonly used in the approaches to localized plastic deformation at finite deformations, see for instance References $[29,31-33]$. This tensor is posed to be equivalent to the average of the RVE deformation gradient $\langle\boldsymbol{F}\rangle$ as

$$
\boldsymbol{I}+\frac{1}{h_{0}} \llbracket \widehat{\boldsymbol{\varphi}} \rrbracket \otimes \widehat{\boldsymbol{N}} \equiv\langle\boldsymbol{F}\rangle=\frac{1}{V_{0}} \int_{\partial \mathcal{B}_{0}} \boldsymbol{\varphi} \otimes \boldsymbol{N} \mathrm{d} A
$$

Hereby, in order to obtain the RVE average in terms of a surface rather than a volume average, the canonical relation ${ }_{1}^{1}$ is used.

4.1.2 Traction. In order to relate the macroscopic traction vector across the interface to the underlying average RVE stress, we employ the Cauchy theorem in the form $(4)_{2}$. With this, the average RVE stress $\langle\boldsymbol{P}\rangle$ enters the macro traction across the interface as follows:

$$
\widehat{\boldsymbol{t}_{0}}=\langle\boldsymbol{P}\rangle \cdot \widehat{\boldsymbol{N}}, \quad\{\widehat{\boldsymbol{P}}\} \equiv\langle\boldsymbol{P}\rangle=\frac{1}{V_{0}} \int_{\partial \mathcal{B}_{0}} \boldsymbol{t}_{0} \otimes \boldsymbol{X} \mathrm{d} A .
$$

4.1.3 Virtual work. The Hill condition requires the average virtual work in the interface to equal the product of average stress and average virtual deformation gradient. For the micromorphic continuum, the

\footnotetext{
${ }^{1}$ The following auxiliary relations are commonly utilized to convert the averaging theorems from volume to surface integrals (e.g. [35,36]) $\boldsymbol{F}=\operatorname{Div}(\boldsymbol{\varphi} \otimes \boldsymbol{I}) \quad \boldsymbol{P}^{\mathrm{t}}=\operatorname{Div}(\boldsymbol{X} \otimes \boldsymbol{P}) \quad \boldsymbol{P}: \boldsymbol{F}=\operatorname{Div}(\boldsymbol{\varphi} \cdot \boldsymbol{P})$
} 
virtual work comprises contributions of all stress measures, while the meso stress and the meso deformation gradient shall be related to the macro level:

$$
\langle\boldsymbol{P}\rangle:\langle\delta \boldsymbol{F}\rangle \equiv\langle\boldsymbol{P}: \delta \boldsymbol{F}+\overline{\boldsymbol{P}}: \delta \overline{\boldsymbol{F}}+\overline{\boldsymbol{Q}}: \cdot \delta \overline{\boldsymbol{G}}\rangle .
$$

With the kinematics (12) and the traction (13), we obtain the the macroscopic virtual work performed in the interface as:

$$
\widehat{\boldsymbol{t}_{0}} \cdot \llbracket \delta \widehat{\boldsymbol{\varphi}} \rrbracket \equiv h_{0}\langle\boldsymbol{P}: \delta \boldsymbol{F}+\overline{\boldsymbol{P}}: \delta \overline{\boldsymbol{F}}+\overline{\boldsymbol{Q}}: \delta \overline{\boldsymbol{G}}\rangle=\frac{1}{w_{0}} \int_{\partial \mathcal{B}_{0}} \boldsymbol{t}_{0} \cdot \delta \boldsymbol{\varphi}+\overline{\boldsymbol{t}}_{0}: \delta \overline{\boldsymbol{F}} \mathrm{d} A .
$$

The scale transition is not complete yet without appropriate boundary conditions, which follow next.

\subsection{Boundary conditions}

The boundary conditions to be imposed on the RVE ensure a correct scale transition if they meet the Hill condition, also denoted as macro-homogeneity condition in reference [36]. The aspired boundary conditions shall in particular account for the two deformation modes, which can occur in the interface, i. e. relative shear and normal tensile opening. For further details also refer to Reference [15].

4.2.1 Deformation boundary conditions. We propose the following linear deformation boundary conditions for the meso and the micro deformation map,

$$
\delta \boldsymbol{\varphi} \equiv\left[\frac{1}{h_{0}} \llbracket \delta \widehat{\boldsymbol{\varphi}} \rrbracket \otimes \widehat{\boldsymbol{N}}\right] \cdot \boldsymbol{X} \quad \forall \boldsymbol{X} \in \partial \mathcal{B}_{0}^{\varphi}, \quad \delta \overline{\boldsymbol{F}} \equiv \mathbf{0} \quad \forall \boldsymbol{X} \in \partial \mathcal{B}_{0}^{\bar{F}}
$$

for a given current macro interface separation. These boundary conditions are admissible as shown in Appendix A.1. For the given RVE of height $h_{0}$ described in Section 3.1, the prescribed meso deformation $(16)_{1}$ obeys the simplified expression for the meso deformation at the top and bottom boundary:

$$
\delta \boldsymbol{\varphi} \equiv \pm \frac{1}{2} \llbracket \delta \widehat{\varphi} \rrbracket \quad \forall \boldsymbol{X} \in \partial \mathcal{B}_{0}^{\varphi \pm}
$$

whereby the identities $\widehat{\boldsymbol{N}} \cdot \boldsymbol{X}=h_{0} / 2 \forall \boldsymbol{X} \in \partial \mathcal{B}_{0}^{\mathrm{T}}, \widehat{\boldsymbol{N}} \cdot \boldsymbol{X}=-h_{0} / 2 \forall \boldsymbol{X} \in \partial \mathcal{B}_{0}^{\mathrm{B}}$ are used.

4.2.2 Traction boundary conditions. Constant traction boundary conditions fulfilling the Hill condition (14) can be imposed on the micromorphic representative volume element as follows

$$
\boldsymbol{t}_{0} \equiv\{\widehat{\boldsymbol{P}}\} \cdot \boldsymbol{N} \quad \forall \boldsymbol{X} \in \partial \mathcal{B}_{0}^{P}, \quad \overline{\boldsymbol{t}}_{0} \equiv \mathbf{0} \quad \forall \boldsymbol{X} \in \partial \mathcal{B}_{0}^{\bar{Q}} .
$$

This is shown in Appendix A.2.

4.2.3 Periodic boundary conditions. The standard periodic deformation boundary conditions that go along with antiperiodic tractions, for a micromorphic RVE read (see Hirschberger et al. [14]):

$$
\begin{array}{rlll}
\delta \boldsymbol{\varphi}^{+}-\delta \boldsymbol{\varphi}^{-}=\mathbf{0} & \forall \boldsymbol{X} \in \partial \mathcal{B}_{0}^{\varphi \pm}, & \boldsymbol{t}_{0}^{+}+\boldsymbol{t}_{0}^{-}=\mathbf{0} & \forall \boldsymbol{X} \in \partial \mathcal{B}_{0}^{\varphi \pm} \\
\delta \overline{\boldsymbol{F}}^{+}-\delta \overline{\boldsymbol{F}}^{-}=\mathbf{0} & \forall \boldsymbol{X} \in \partial \mathcal{B}_{0}^{\bar{F} \pm}, & \overline{\boldsymbol{t}}_{0}^{+}+\overline{\boldsymbol{t}}_{0}^{-}=\mathbf{0} & \forall \boldsymbol{X} \in \partial \mathcal{B}_{0}^{\bar{F}} .
\end{array}
$$

Herein the positive boundary $\partial \mathcal{B}_{0}^{+}$comprises the top and the right edge, while the left and the bottom edge represent the negative boundary $\partial \mathcal{B}_{0}^{-}$, analogously to the literature [35,37]. The admissibility of these 
4.2.4 Hybrid boundary conditions. To account for the deformation modes and the geometry of the material layer, at the top and bottom boundaries of the RVE, which are conceptually aligned with the positive and negative edges of the interface, $\widehat{\Gamma}_{0}^{+}$and $\widehat{\Gamma}_{0}^{-}$, respectively, we fully prescribe the meso-deformation boundary conditions by means of the macro interface separation $\llbracket \hat{\boldsymbol{\varphi}} \rrbracket$ as

$$
\delta \boldsymbol{\varphi}(\boldsymbol{X})=\frac{1}{2} \llbracket \delta \widehat{\boldsymbol{\varphi}} \rrbracket \quad \forall \boldsymbol{X} \in \partial \mathcal{B}_{0}^{\varphi \mathrm{T}}, \quad \delta \boldsymbol{\varphi}(\boldsymbol{X})=-\frac{1}{2} \llbracket \delta \widehat{\boldsymbol{\varphi}} \rrbracket \quad \forall \boldsymbol{X} \in \partial \mathcal{B}_{0}^{\varphi \mathrm{B}}
$$

as examined for the uniform displacement boundary conditions (17). For the micro deformation map, the trivial boundary condition (16)b must be fulfilled on the top as well as on the bottom

$$
\delta \overline{\boldsymbol{F}}(\boldsymbol{X})=\mathbf{0} \quad \forall \boldsymbol{X} \in \partial \mathcal{B}_{0}^{\varphi \mathrm{T}} \cup \partial \mathcal{B}_{0}^{\varphi \mathrm{B}}
$$

Consequently, we choose the micro deformation map to remain as the identity: $\overline{\boldsymbol{F}}=\mathbf{1}$ at the top and bottom boundaries. In the plane of the interface, periodic meso and micro deformations, which encounter antiperiodic tractions and double tractions, are applied as presented in Equations (19)-(20). Thus the periodic deformation and antiperiodic traction boundary condition in plane specify to be formulated with respect to the left and right corner as:

$$
\begin{array}{rlll}
\delta \boldsymbol{\varphi}^{\mathrm{R}}-\delta \boldsymbol{\varphi}^{\mathrm{L}}=\mathbf{0} & \forall \boldsymbol{X} \in \partial \mathcal{B}_{0}^{\varphi \mathrm{L} / \mathrm{R}}, & \boldsymbol{t}_{0}^{\mathrm{R}}+\boldsymbol{t}_{0}^{\mathrm{L}}=\mathbf{0} & \forall \boldsymbol{X} \in \partial \mathcal{B}_{0}^{\varphi \mathrm{L} / \mathrm{R}}, \\
\delta \overline{\boldsymbol{F}}^{\mathrm{R}}-\delta \overline{\boldsymbol{F}}^{\mathrm{L}}=\mathbf{0} & \forall \boldsymbol{X} \in \partial \mathcal{B}_{0}^{\bar{F} \mathrm{~L} / \mathrm{R}}, & \overline{\boldsymbol{t}}_{0}^{\mathrm{R}}+\overline{\boldsymbol{t}}_{0}^{\mathrm{L}}=\mathbf{0} & \forall \boldsymbol{X} \in \partial \mathcal{B}_{0}^{\bar{F} \mathrm{~L} / \mathrm{R}} .
\end{array}
$$

This choice of boundary conditions can be shown to be admissible with respect to the Hill condition (14), see Appendix A.4.

\section{Computational homogenization}

With the theoretical multiscale framework presented, we now strive for a finite-element based multiscale procedure to efficiently solve boundary value problems which incorporate the both the meso heterogeneity and the intrinsic microstructure within the material layer. Therefore we avail ourselves of computational homogenization, as for instance proposed in References [16,18], in which a nested finite element solution is performed at both the macro and the meso level. In our approach, cohesive interface elements situated between continuum elements represent the material layer at the macro level, as already proposed in Reference [8]. While the constitutive behaviour of the surrounding bulk is postulated, the constitu- 

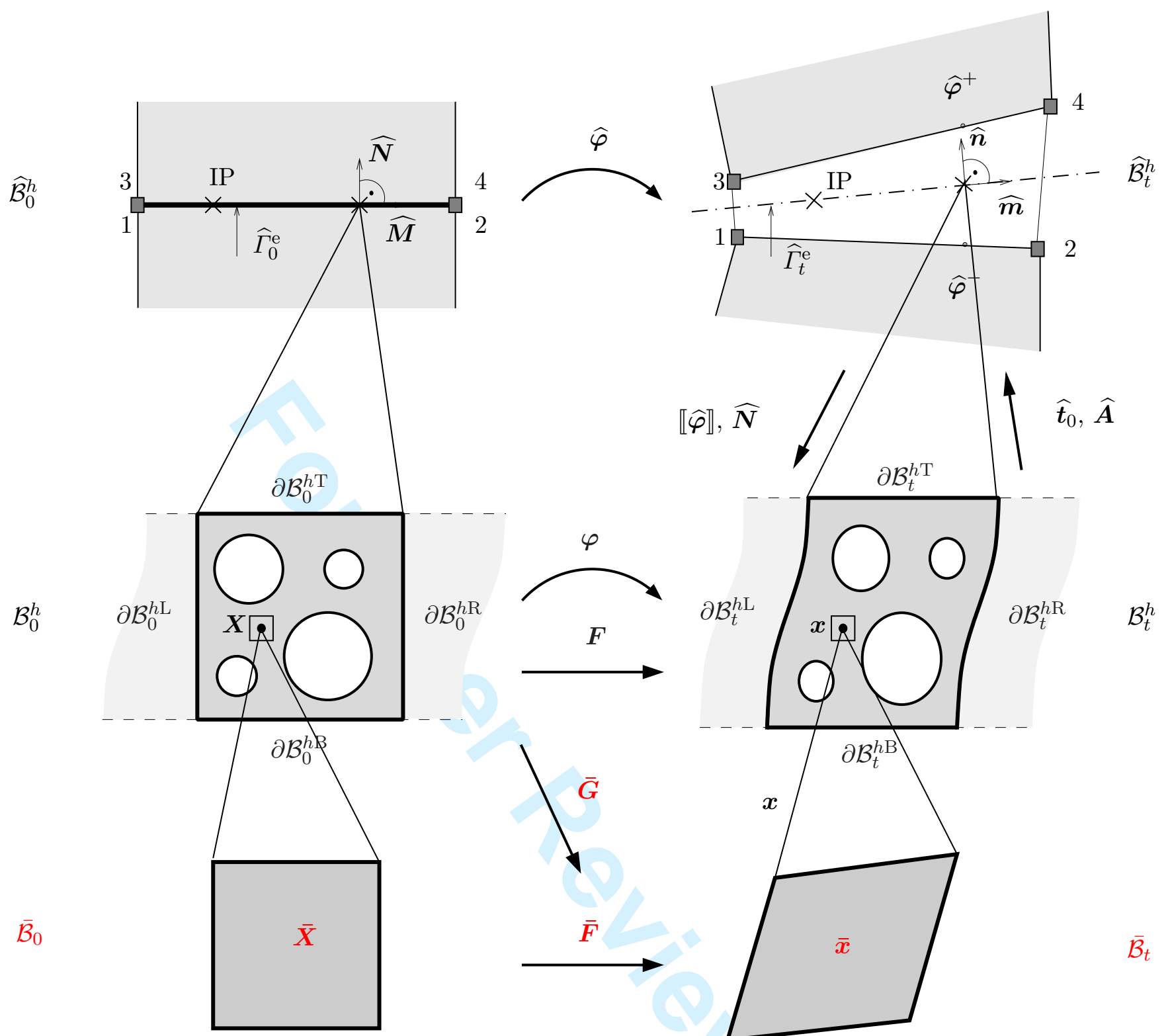

$\overline{\mathcal{B}}_{0}$

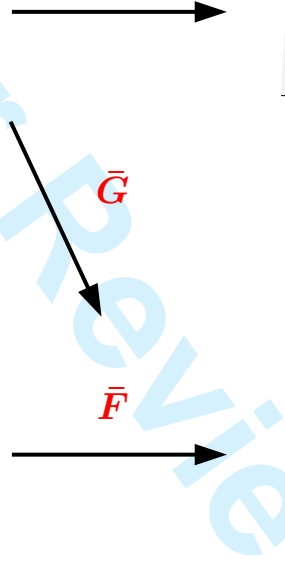

\footnotetext{
Figure 6. Computational homogenization between the interface integration point (IP) of the interface element on $\widehat{\Gamma}_{0}^{\mathrm{e}}$ at the macro level and the underlying representative volume element $\mathcal{B}_{0}^{h}$ with boundary $\partial \mathcal{B}_{0}^{h}$ and microcontinua $\overline{\mathcal{B}}_{0}$ providing additional micro degrees of freedom at each mesocontinuum point $\boldsymbol{X}$.
}

tive behaviour or rather the traction-separation relation (6) of the interface element is obtained from the underlying micromorphic mesostructure. Therefore, within a deformation-driven computational homogenization in particular the macro cohesive traction vector and the macro tangent operator at each interface element integration point need to be obtained from the representative volume element during the iterative solution.

\section{$5.1 \quad$ Nested multiscale solution scheme}

For the iterative solution of the nonlinear multiscale boundary value problem, a nested multiscale solution scheme along the lines of References [22,35,37] is applied. In extension to the scheme presented in Reference [8], the procedure illustrated in Figure 6, here involves both the macro and the meso level explicitly as well as the micro level implicitly. At each integration point of each interface element, the macro jump $\llbracket \hat{\varphi} \rrbracket$ is evaluated, being zero initially. It is applied as boundary condition to the respective micromorphic RVE finite-element mesh. With the RVE's stiffness matrix and the residual vector, both the macroscopic 
tangent operator $\widehat{\boldsymbol{A}}$ and the macroscopic traction vector $\widehat{\boldsymbol{t}}_{0}$ of this interface integration point are computed and stored. This procedure is repeated for each integration point of each interface element during each macro iteration step.

\section{$5.2 \quad$ Numerical solution of the RVE problem}

For the solution of the RVE boundary value problem in $\mathcal{B}_{0}^{h}$ involving the micromorphic continuum, the numerical framework for the micromorphic continuum presented by Hirschberger et al. [14,15] is exploited. The primary unknown variables at the finite-element nodes are the meso deformation map $\boldsymbol{\varphi}_{K}$ and the micro deformation map $\overline{\boldsymbol{F}}_{L}$. In general, different orders of approximation will be used for these two, and thus we denote the nodes with unknown meso deformation map with $I$ and $K$, whereas those with unknown micro deformation map with $J$ and $L$. A coupled problem is obtained, as recalled here in its linearized format:

$$
\sum_{I, J} \sum_{K, L}\left[\begin{array}{cc}
\mathbf{K}_{I K}^{\varphi \varphi} & \mathbf{K}_{I L}^{\varphi \bar{F}} \\
\mathbf{K}_{J K}^{\bar{F} \varphi} & \mathbf{K}_{K L}^{\bar{F} \bar{F}}
\end{array}\right] \cdot\left[\begin{array}{c}
\Delta \boldsymbol{\varphi}_{K} \\
\Delta \overline{\boldsymbol{F}}_{L}
\end{array}\right]=\sum_{I, J}\left[\begin{array}{c}
\Delta \mathbf{f}_{0 I} \\
\Delta \overline{\mathbf{f}}_{0 J}
\end{array}\right]
$$

The spatial residual vector $\left[\mathbf{f}_{0 I}, \overline{\mathbf{f}}_{0 J}\right]^{\mathrm{t}}$ contains the nodal forces $\mathbf{f}_{0 I}$ resulting from the tractions as well as the nodal double forces $\overline{\mathbf{f}}_{0 J}$ stemming from the double tractions.

5.2.1 Boundary conditions on the RVE. We make use of the hybrid boundary conditions on the RVE as introduced in Section 4.2.4, which account both quantitatively and qualitatively for the interface deformation. For the periodicity of the deformation along the interface, the RVE finite-element mesh is subjected to the restriction that the left and the right-hand side boundary, $\partial \mathcal{B}_{0}^{h^{\mathrm{L}}}$ and $\partial \mathcal{B}_{0}^{h^{\mathrm{R}}}$, respectively, have the same arrangement of nodes. This periodicity is enforced by a transformation of the system of equations into a system in terms of the independent degrees of freedom only, as suggested in References [37]. These independent degrees of freedom cover all degrees of freedom of the boundary nodes at the top, bottom and left boundary as well as those of the interior nodes. The remaining right boundary nodes represent the dependent degrees of freedom. The macro deformation (21)-(22) is applied incrementally on all top and bottom nodes of the RVE within the reduced system of equations.

5.2.2 Solution and update of the degrees of freedom. During each macro Newton-Raphson iteration step, within the respective RVE, the reduced system of equations enveloping the independent degrees of freedom is iteratively solved using a Newton-Raphson scheme. Once equilibrium is found, we recompute the dependent degrees of freedom and store the entire current (or rather spatial) RVE meso and micro degrees of freedom.

\subsection{Homogenized interface quantities}

Since we want to extract the sought-for macroscopic quantities, i.e. the traction vector $\widehat{\boldsymbol{t}}_{0}$ and the tangent operator $\widehat{\boldsymbol{A}}$, from the system of equations on the RVE or rather meso level is condensed such that only the stiffness matrix and the residual in terms of the prescribed degrees of freedom remain.

5.3.1 Macroscopic traction vector. The macro traction vector is obtained based on the averaging (13) which is rewritten is the discrete version as

$$
\widehat{\boldsymbol{t}}_{0}=\frac{1}{V_{0}} \sum_{I} \mathbf{f}_{0 I} \otimes \boldsymbol{X}_{I} \cdot \widehat{\boldsymbol{N}} \quad I \in \partial \mathcal{B}^{h \mathrm{~B}} \cup \partial \mathcal{B}^{h \mathrm{~T}}
$$


The summation runs over all boundary nodes $I$ the deformation is prescribed at.

5.3.2 Macroscopic tangent operator. With the relation between the macro and meso kinematics $(12)_{1}$ and the deformation boundary conditions (21)-(22), the tangent operator (6) is extracted from a summation over all prescribed nodes $I, K$ :

$$
\widehat{\boldsymbol{A}}=\frac{1}{w_{0} h_{0}^{2}}[\widehat{\boldsymbol{N}} \otimes \widehat{\boldsymbol{N}}]:\left[\sum_{I} \sum_{K}\left[\boldsymbol{X}_{K} \otimes \boldsymbol{X}_{I}\right]: \mathbf{K}_{I K}^{\varphi \varphi}\right], \quad I, K \in \partial \mathcal{B}^{h \mathrm{~B}} \cup \partial \mathcal{B}^{h \mathrm{~T}}
$$

See Appendix B to follow the derivation of this tangent in more detail.

These quantities provide the sought-for constitutive information in each interface integration point in the macro boundary value problem, which enable to pursue the iterative macro solution.

\section{Numerical examples}

The framework of the computational homogenization with the interface element at the macro level and the micromorphic RVE on the meso and micro level is applied to numerical examples, which shall display the successful implementation of the proposed computational multiscale framework and thereby particularly reveal the framework's capability to simulate size effects of the intrinsic microstructure in the material layer on the macroscopic response. To this end, we investigate the influence of the choice of the additional micromorphic material parameters within the RVE on the macro response. Since for homogeneous deformations, the micro deformation would not be excited at all, we primarily choose RVE geometries that introduce some kind of meso heterogeneity, which in a rather straightforward case is a void.

First, in two benchmark-type examples with a simplified macro boundary value problem, we display the characteristics of the micromorphic RVE subject to the proposed hybrid boundary conditions under shearing and tensile mode loading. Besides the resulting traction-separation laws, we show the Cauchytype meso stress field in the RVE for the different sets of micromorphic parameters to provide a reference for future studies. With these benchmark-type results at hand, we move on to two multiscale problems with a more complex macrostructures in order to show the capability of the model to in fact solve macro boundary value problems using the additional information on the material layer material provided through the RVE.

A finite-element simulation is performed on both the macro and the meso level, with the boundary value problem described for the macro scale and the constitutive response evaluated on the RVE. At the macro level, we use four-node plane-strain elements with bi-linear interpolations for the bulk as well as interface elements with four nodes and two Gaussian integration points. For the micromorphic RVE we utilize Lagrangian plane-strain elements with bi-quadratic interpolation for both the meso placement $\boldsymbol{X}$ and the meso deformation map $\boldsymbol{\varphi}$, as well as bi-linear interpolation for the micro deformation map $\overline{\boldsymbol{F}}$. Throughout the section, the components of the respective displacement jumps and tractions will be expressed in terms of the orthonormal basis $\boldsymbol{M}, \boldsymbol{N}$ shown in Figure 2.

\subsection{Interface under fully prescribed shear mode loading}

As a benchmark problem, we first consider a single interface element, which is subject to shear mode loading, as depicted in Figure 7. The deformation of the surrounding bulk is fully controlled so that no degrees of freedom are activated there. The RVE is modelled as a square specimen with a circular centred hole of $20 \%$ void fraction, meshed with 96 micromorphic elements. Within the RVE of height $h_{0}$, the sample material parameters, i.e. Young's modulus $E=1000$ and Poisson's ratio $\nu=0.3$ are chosen for all computations. At first, the internal length is varied for a fixed scale-transition parameter, $p / E=10$, see Section 6.1.1. Secondly, in Section 6.1.2 the scale-transition parameter is varied for a fixed internal length of $l=0.2 h_{0}$. 


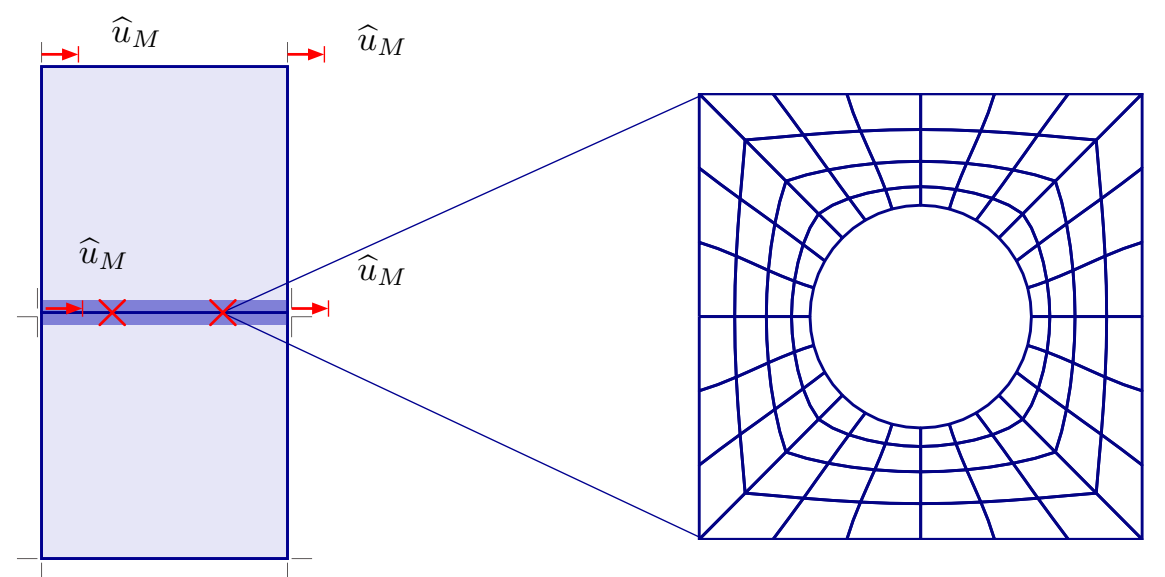

Figure 7. Example 6.1: Benchmark problem for multiscale framework: Single interface element subjected to shear.

6.1.1 Influence of the micromorphic internal length - size dependence. The traction-separation curves at an integration point in the interface element are shown for different values of the micromorphic internal-length parameter related to the total height of the interface in Figure 9. The traction-separation curve in loading direction in Figure $9(\mathrm{a}), \widehat{t}_{\widehat{0}}$ vs. $\llbracket \widehat{\varphi}_{\widehat{M}} \rrbracket$, obeys an approximately linear relation. Moreover a strong size dependence is observed in the sense that larger internal-length parameters yield a notably stiffer response. The traction-separation curve, $\widehat{t}_{0 \widehat{N}}$ vs. $\llbracket \widehat{\varphi}_{\widehat{M}} \rrbracket$, perpendicular to the loading direction shown in Figure 9(b) reveals a coupled response. The tangential tractions are much smaller than the normal components, nevertheless a nonlinear relation is observed. The dependence of the internal length is reverse compared to the first curve; here smaller internal length yield the stiffer normal response for the shear loading.

The corresponding spatial configurations with the micromorphic meso stress are displayed in Figure 10. We observe that for increasing internal length, the deformation of the specimen around the hole becomes less pronounced. Moreover, for non-zero internal length we observe the typical non-symmetric meso stress, when looking at the shear components. Especially, the shear component $\sigma_{\widehat{M} \widehat{N}}$ is significantly higher for the largest internal length. Also the normal stress in tangential direction $\sigma_{\widehat{M} \widehat{M}}$ is remarkably larger, while the influence on the other two components is rather weak.

6.1.2 Influence of the micromorphic scale transition parameter. Next, we study the influence of the scale-transition parameter $p$, which controls the kinematic correlation between the meso and the micro scale. For a variation of the parameter $p$ in exponential steps to the basis ten, the spatial meshes of the macro and the mesostructure are presented in Figure 11. The resulting traction-separation curves, $\widehat{t}_{0 \widehat{M}}$ vs. $\llbracket \widehat{\varphi}_{\widehat{M}} \rrbracket$ and $\widehat{t}_{0 \widehat{N}}$ vs. $\llbracket \widehat{\varphi}_{\widehat{M}} \rrbracket$, are shown in Figure 12. First of all, we notice that the response is coupled in the sense that an exclusively shearing deformation also yields normal tractions. As observed before, the shear component $\widehat{t}_{0 \widehat{M}}$ vs. $\llbracket \widehat{\varphi}_{\widehat{M}} \rrbracket$ obeys an approximately linear relation, while the tension response to the shear deformation $\widehat{t}_{0 \widehat{N}}$ vs. $\llbracket \widehat{\varphi}_{\widehat{M}} \rrbracket$ is strongly nonlinear, however significantly smaller. Furthermore, we observe that smaller scale transition parameters yield a stiffer response in the shear traction $\widehat{t}_{0 \widehat{M}}$ vs. $\llbracket \widehat{\varphi}_{\widehat{M}} \rrbracket$ and a weaker response in the normal traction, $\widehat{t}_{0 \widehat{N}}$ vs. $\llbracket \widehat{\varphi}_{0 \widehat{M}} \rrbracket$. The meso stress components are plotted in Figure 13. For larger scale-transition parameters, all stress components becomes more pronounced in the direct vicinity of the hole. 


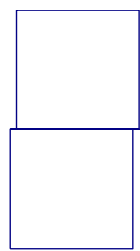

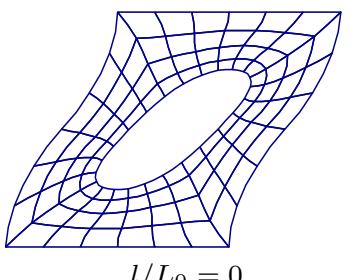

$l / L_{0}=0$

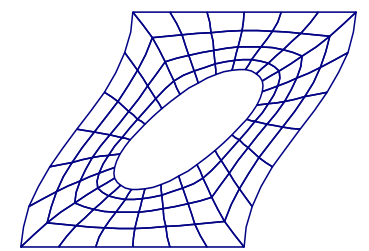

$l / L_{0}=0.1$

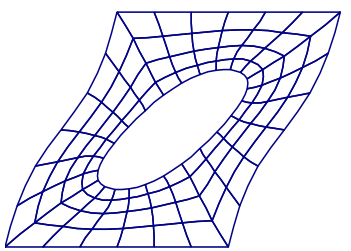

$l / L_{0}=0.2$

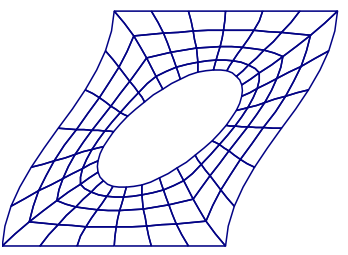

$l / L_{0}=0.4$

Figure 8. Example 6.1: Spatial macro mesh, spatial RVE meshes at different internal-length parameters.
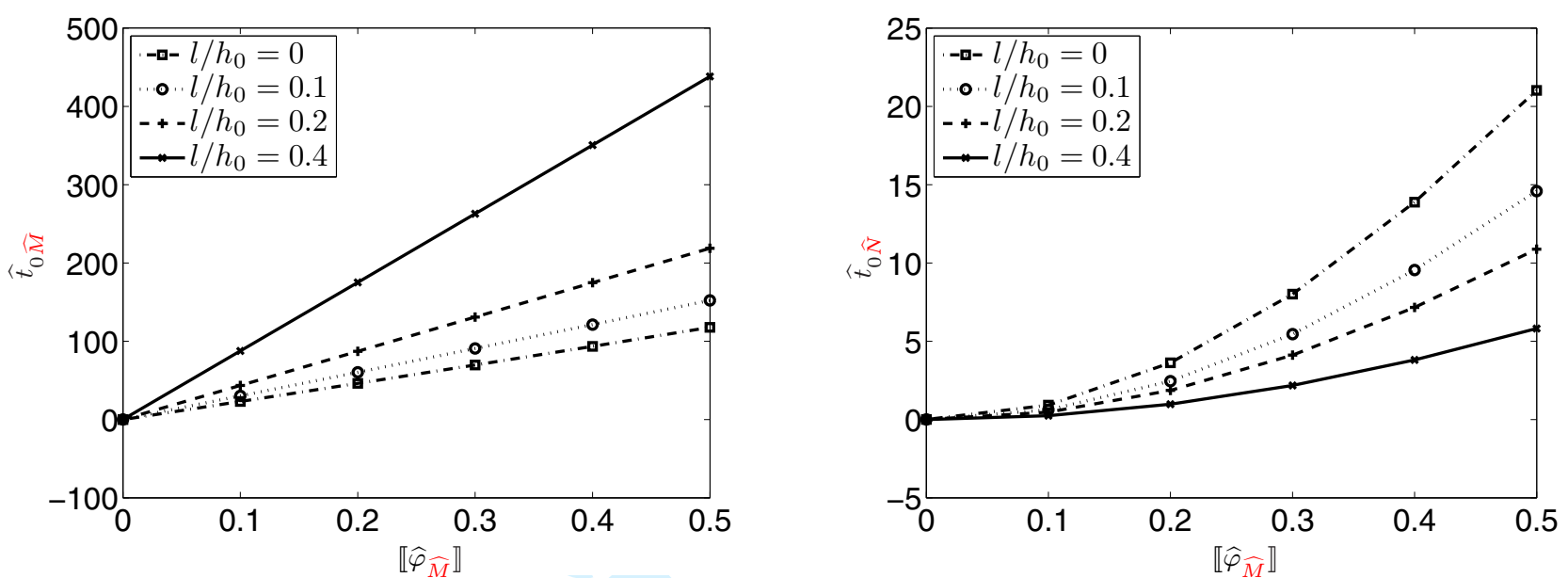

Figure 9. Example 6.1: Traction-separation curves for different internal-length parameters.
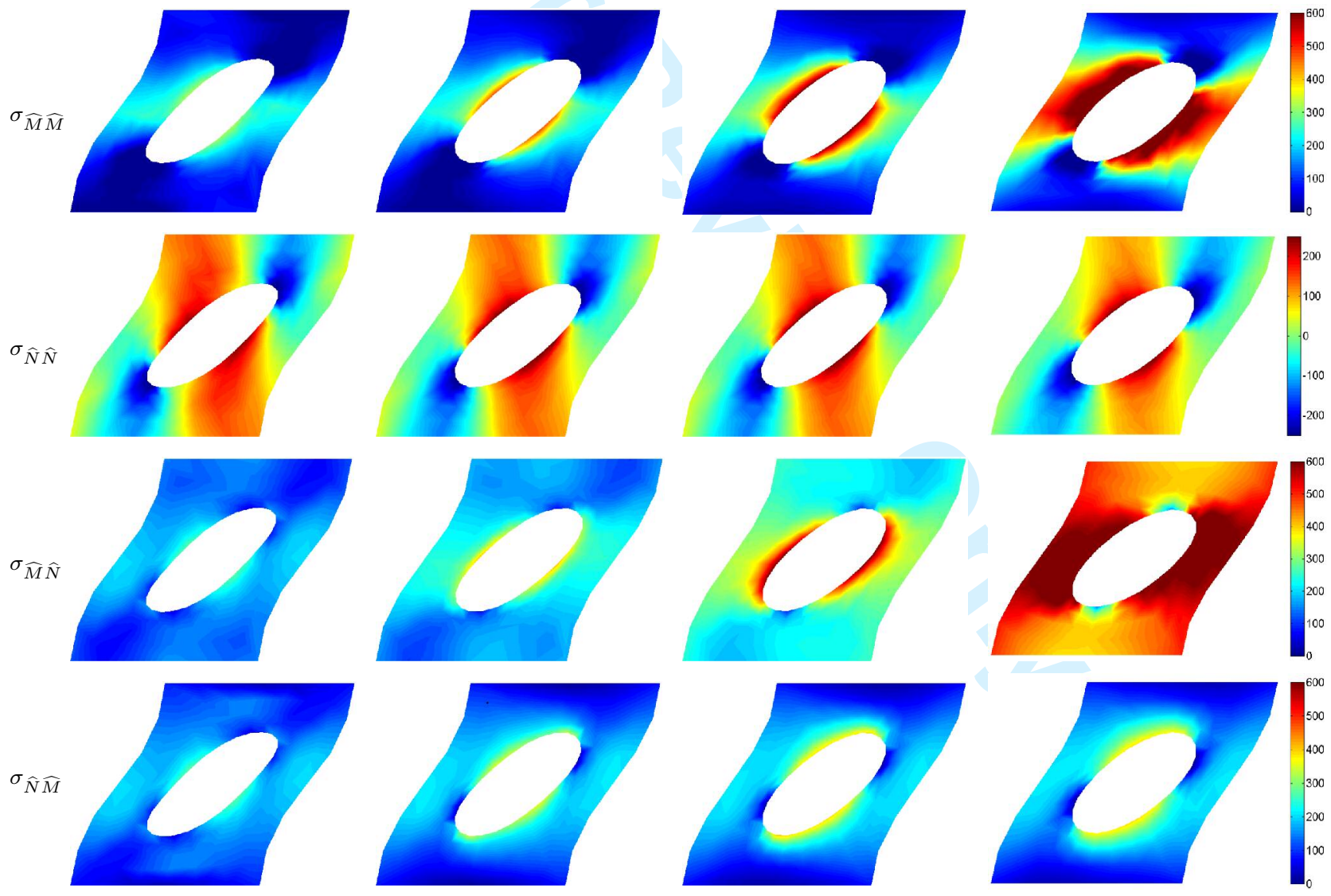

$l / L_{0}=0.2$

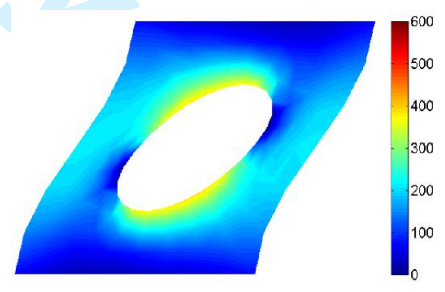

$l / L_{0}=0.4$

Figure 10. Example 6.1: Cauchy-type meso stress components at different internal-length parameters. 
1

2

4

5

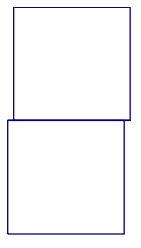

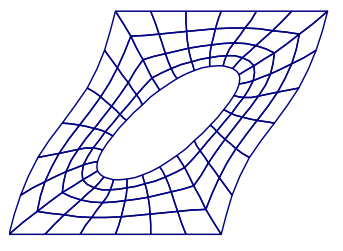

$p / E=0.1$

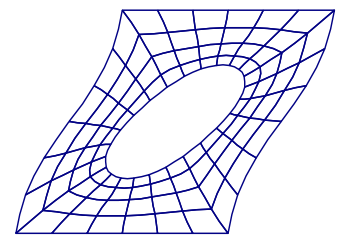

$p / E=1$

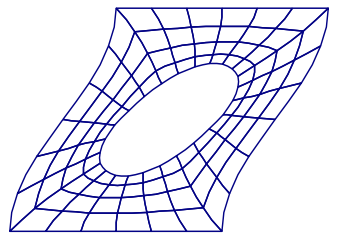

$p / E=10$

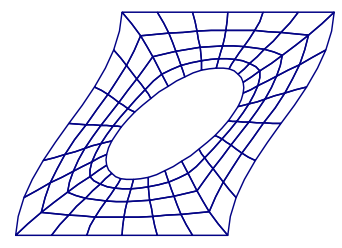

$p / E=100$

Figure 11. Example 6.1: Spatial macro mesh, spatial RVE meshes at different scale-transition parameters.
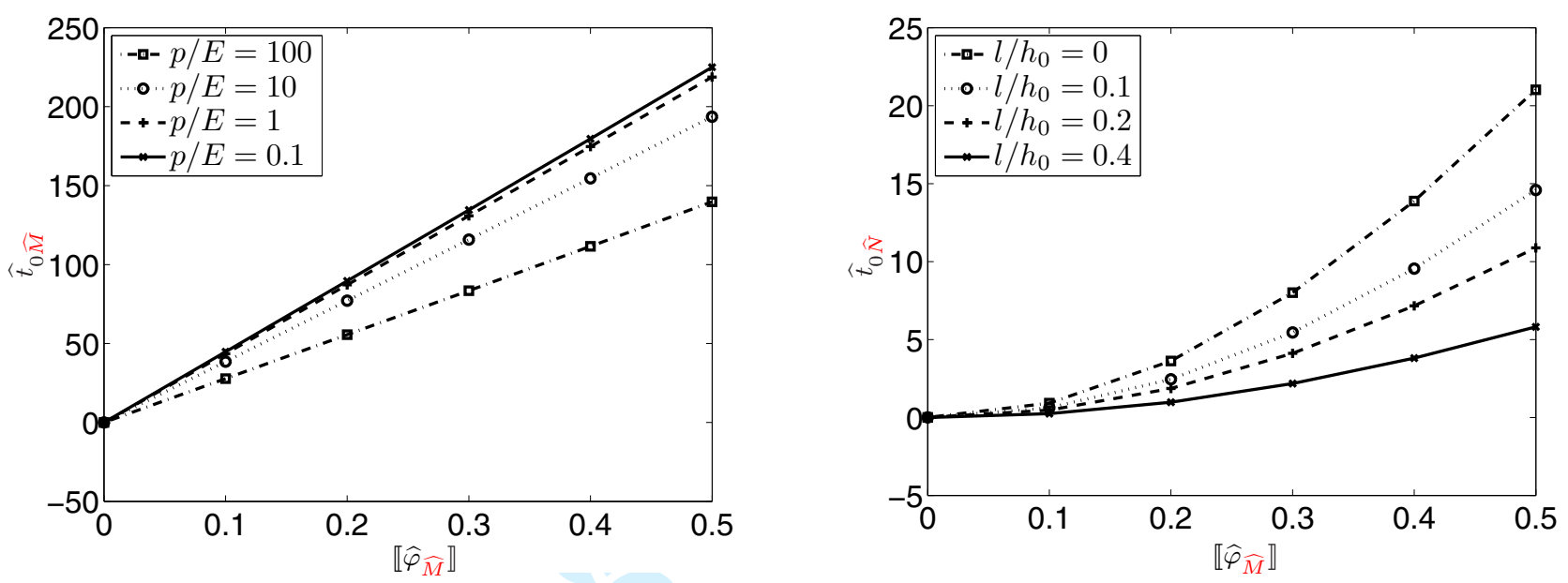

Figure 12. Example 6.1: Macro traction-separation curves for different scale-transition parameters.
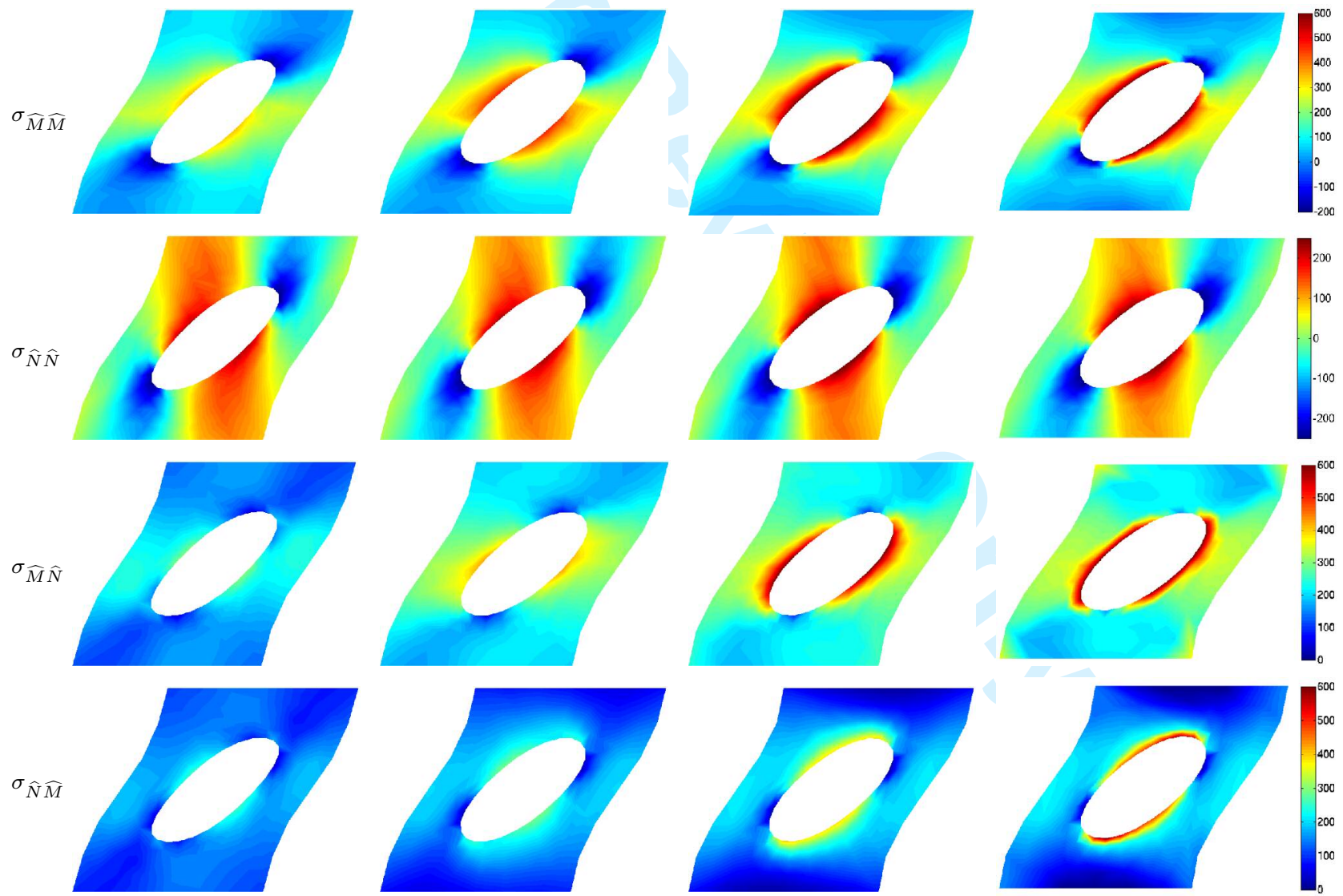

$p / E=1$

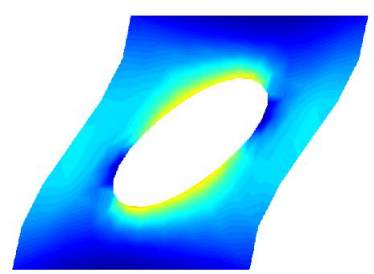

$p / E=10$

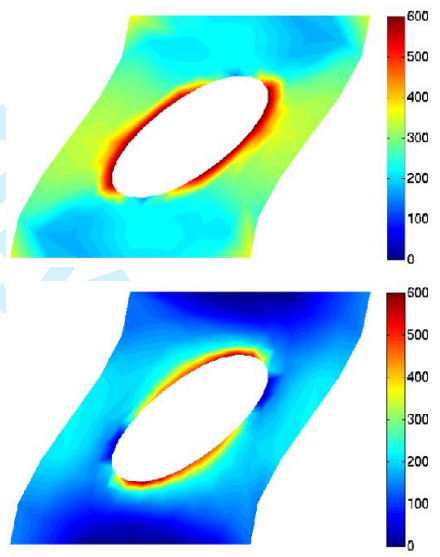

$p / E=100$

Figure 13. Example 6.1: Cauchy-type stress components for different scale-transition parameters 
6.2.1 Influence of the micromorphic internal length - size dependence. For the present tensile-mode loading, the traction-separation curves in loading direction, $\widehat{t}_{0 \widehat{N}}$ vs. $\llbracket \widehat{\varphi}_{0 \widehat{N}} \rrbracket$, are compared for different internal length parameters in Figure 16. This response obeys a nonlinear behaviour, whereby the curves display a stiffer response for larger internal-length parameters $l$. The deformed meso meshes in Figure 15 reflect the same observation: For a larger internal length, the opening of the void is less pronounced which underlines the stiffer continuum response. The particular components of the meso stress of Cauchy type are shown in Figure 19. Again for a larger internal length, the non-symmetry of the Cauchy type meso stress proves to be stronger. However, different from the shear loading, where the distribution of the shear meso stress underwent a significant influence, the qualitative influence is comparatively small for the tension loading at hand.

6.2.2 Influence of the micromorphic scale-transition parameter. The influence of the micromorphic scale-transition parameter $p$ on the macroscopic traction-separation response is plotted in Figure 18. Thereby the parameter is varied in exponential steps to the basis ten. We observe that the specimen with the higher scale-transition parameter $p$ exhibits a stiffer behaviour. This is reflected by the deformed meshes in Figure 17 particularly in the direct vicinity of the hole. In the corresponding Cauchy-type meso stress component, shown in Figure 20, for larger $p$, again the stress is significantly larger at the edge of the hole. This matches well the observations in Reference [14], that for larger scale-transition parameters $p$, the region in which meso deformation map and the deformation gradient deviate from each other are strongly localized to the heterogeneity. 


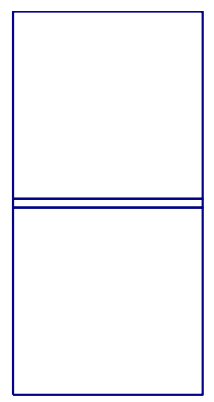

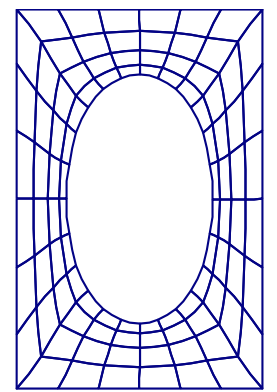

$l / h_{0}=0.0$

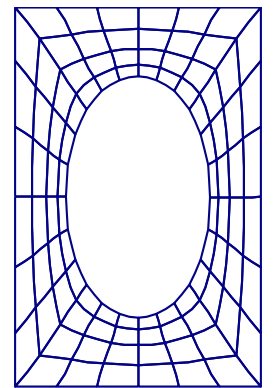

$l / h_{0}=0.1$

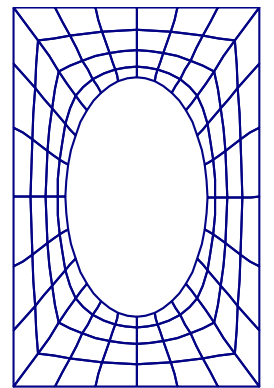

$l / h_{0}=0.2$

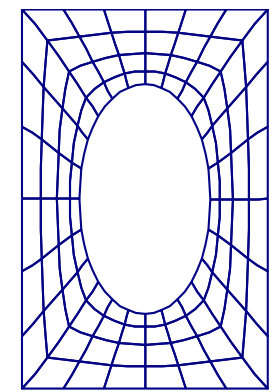

$l / h_{0}=0.4$

Figure 15. Example 6.2: Spatial macro mesh, spatial RVE meshes at different internal-length parameters.
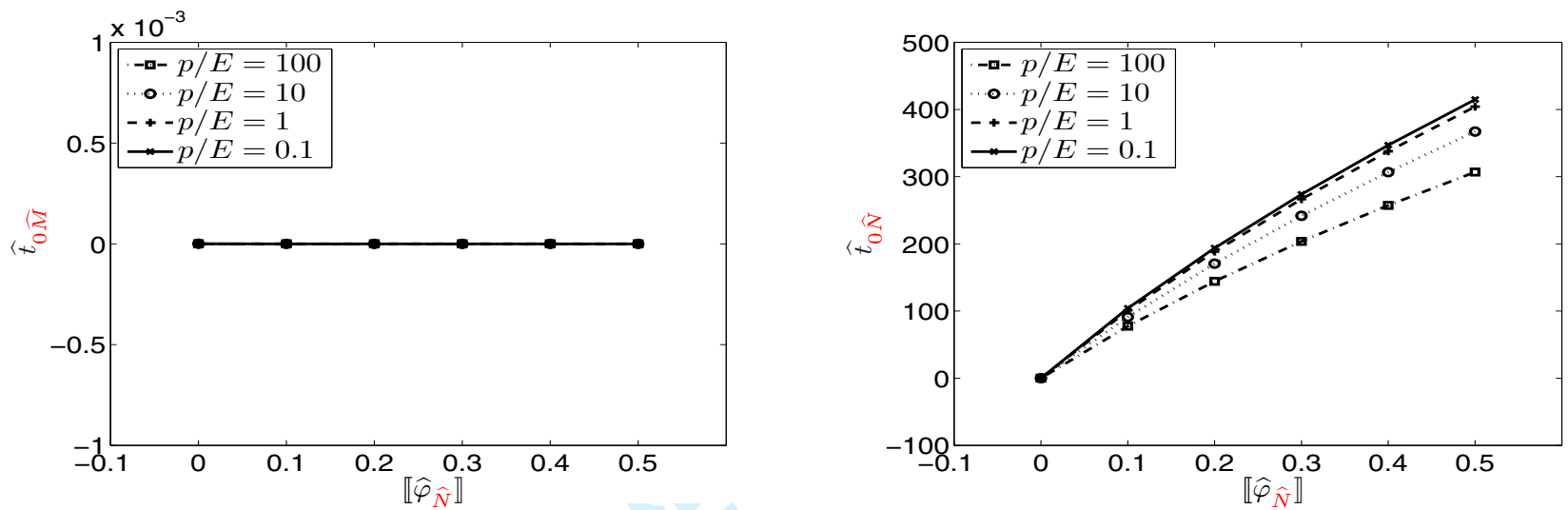

Figure 16. Example 6.2: Traction-separation curves for different internal-length parameters.

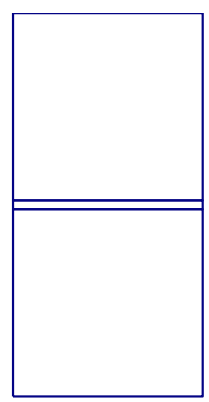

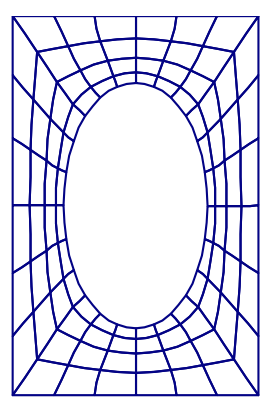

$p / E=100$

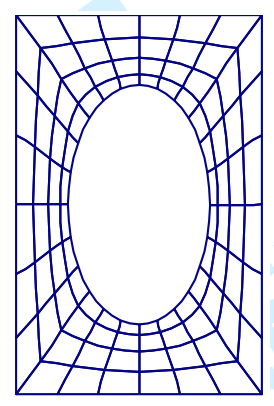

$p / E=10$

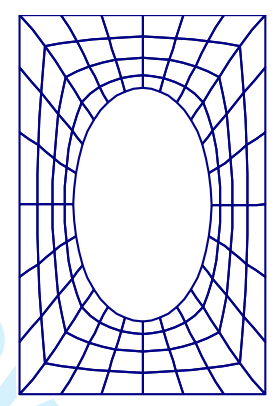

$p / E=1$

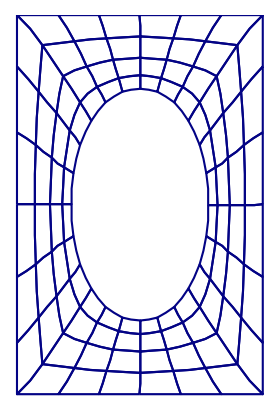

$p / E=0.1$

Figure 17. Example 6.2: Spatial macro mesh, spatial RVE meshes at different scale-transition parameters.
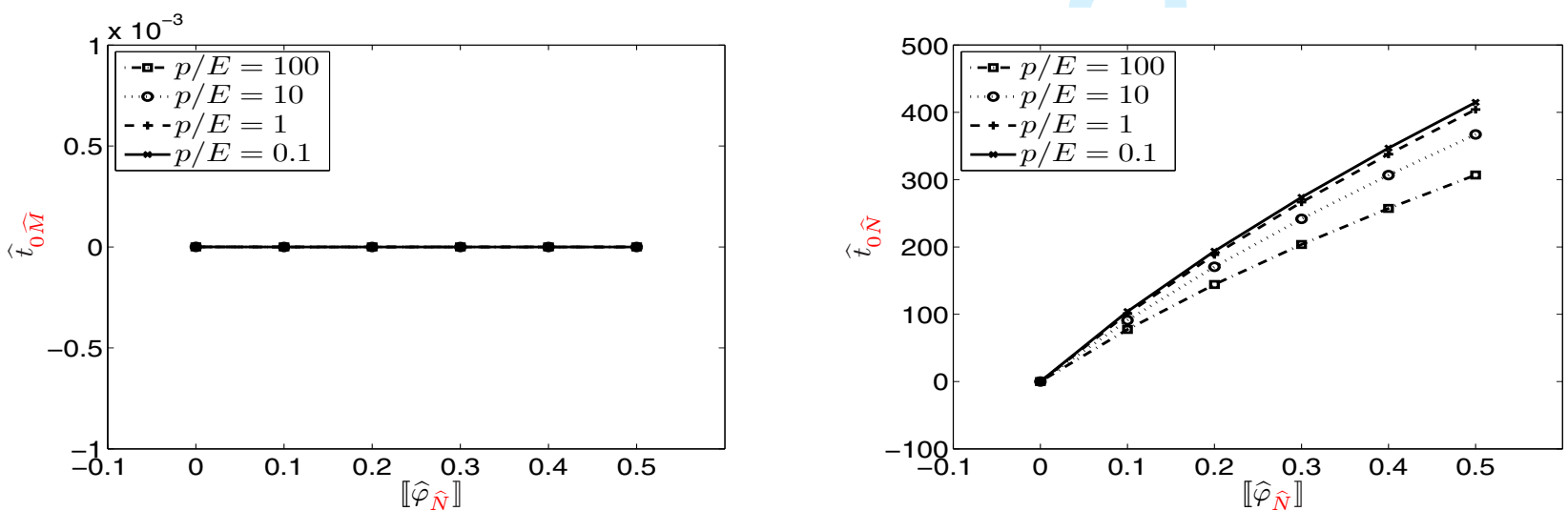

Figure 18. Example 6.2: Macro traction-separation curves for different scale-transition parameters. 

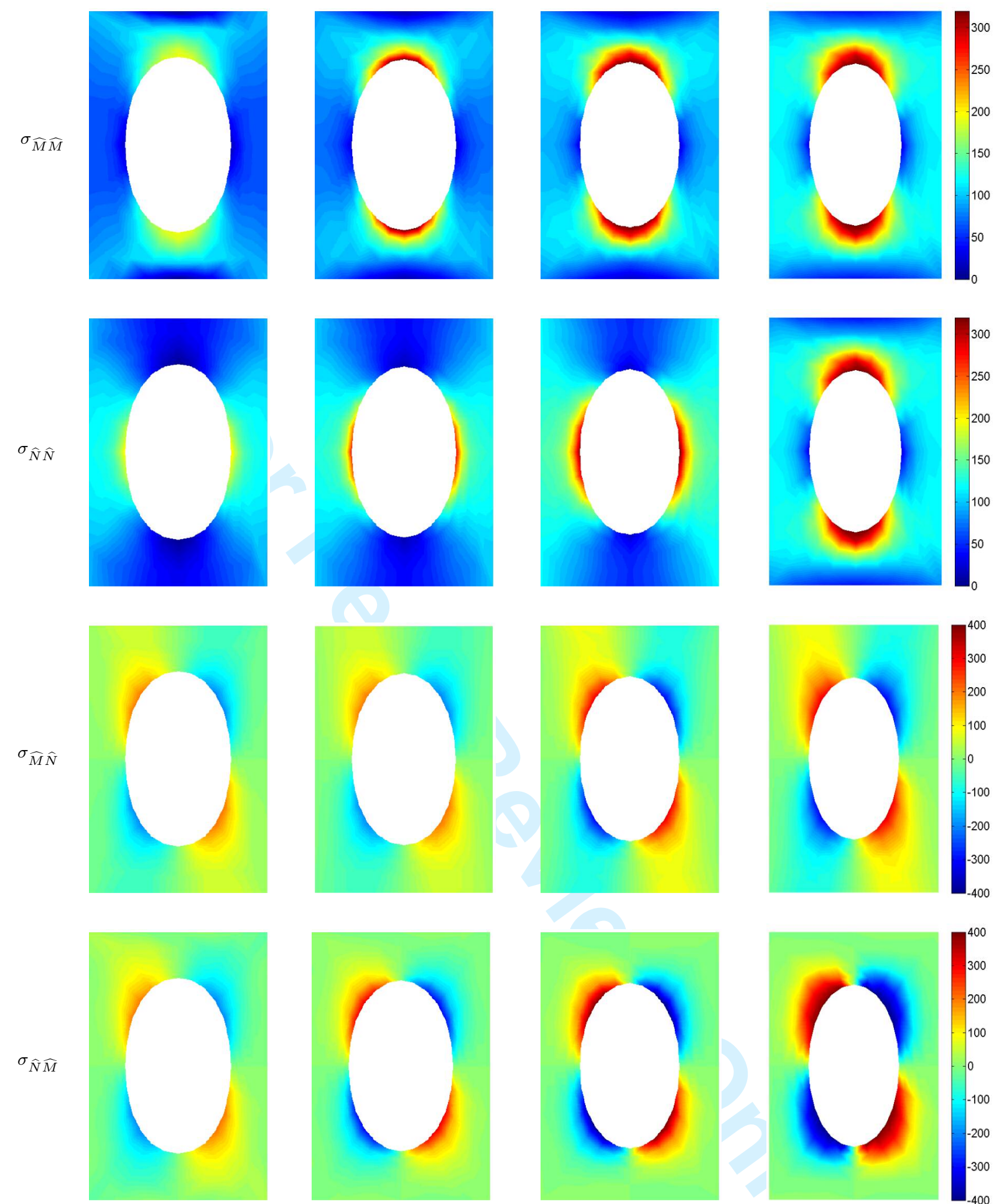

$l / h_{0}=0.0$

$l / h_{0}=0.1$

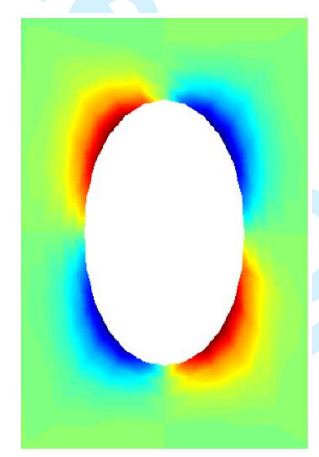

$l / h_{0}=0.2$

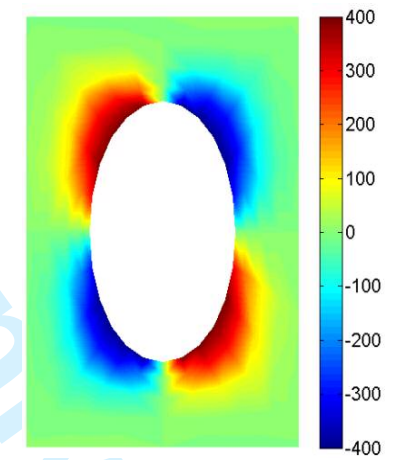

$l / h_{0}=0.4$

Figure 19. Example 6.2: Cauchy-type meso stress components at different internal-length parameters. 
1

2

3

4

5

6

7

8

9

10

11

12

13

14

15

16

17

18

19

20

21

22

23

24

25

26

27

28

29

30

31

32

33

34

35

36

37

38

39

40

41

42

43

44

45

46

47

48

49

50

51

52

53

54

55

56

57

58

59

60
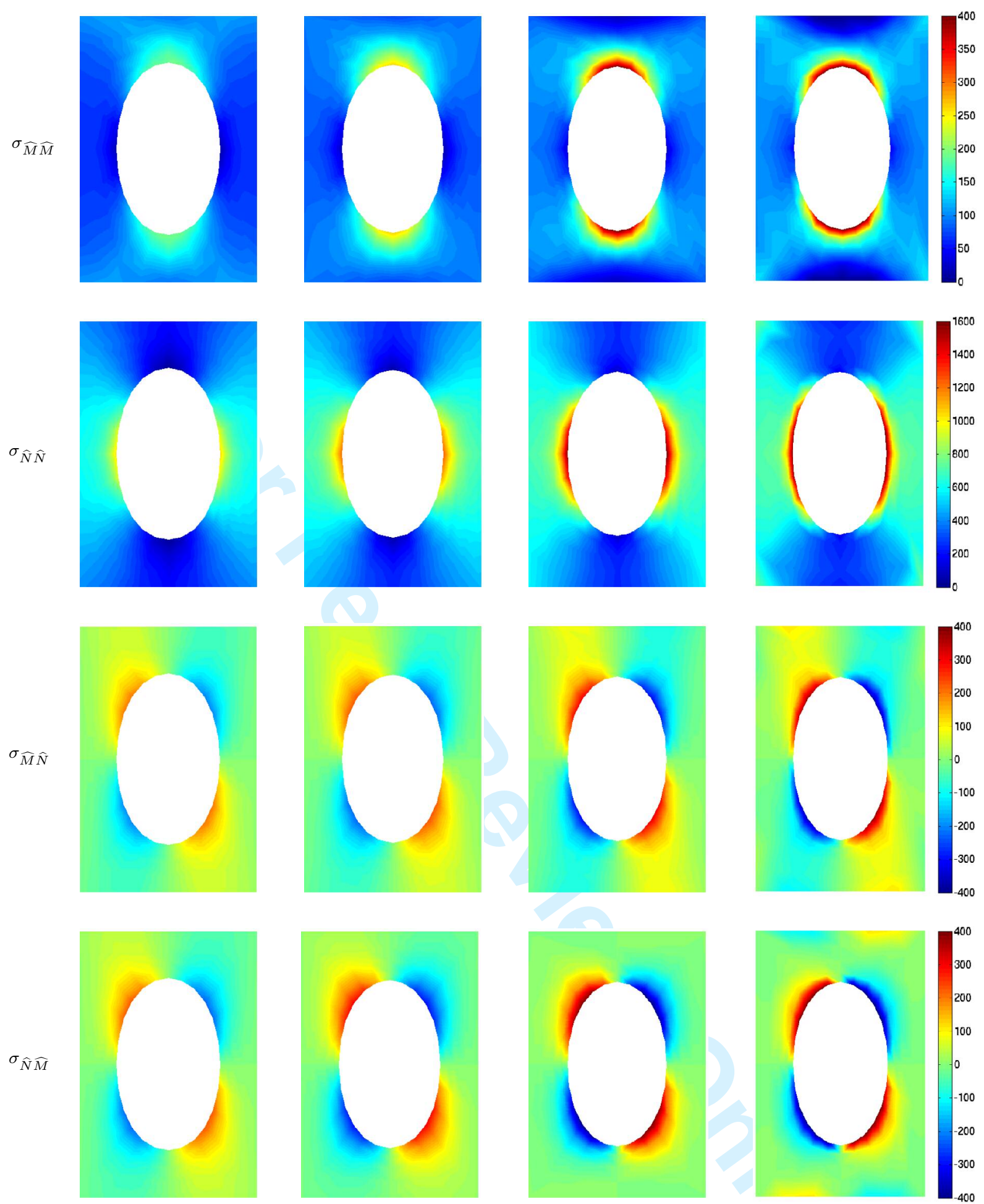

$p / E=0.1$

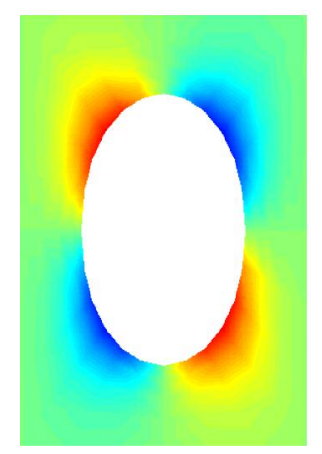

$p / E=1$

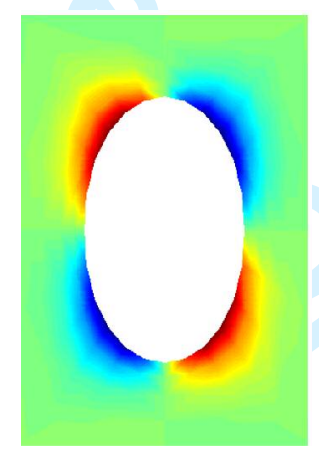

$p / E=10$

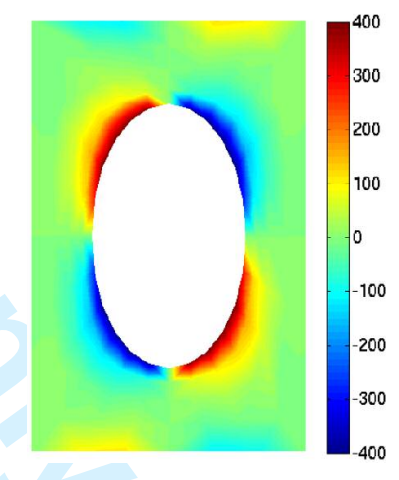

$p / E=100$

Figure 20. Example 6.2: Cauchy-type meso stress components at different scale-transition parameters. 


\subsection{Material layer within an infinite shear layer under shear-dominated mixed-mode loading}

After the benchmark-type examples shown so far, which only considered the micromorphic RVE and the resulting homogenized traction-separation relations, now the macro response is also taken into account.

Therefore at the macro level a material layer within the periodic shear layer is endowed with micromorphic representative volume elements, see Figure 21. Micromorphic microstructures consisting of a square RVE with a lentil-shaped void of horizontal and vertical orientation, respectively, are compared with another specimen with a circular centred hole. Furthermore a homogeneous shear layer is considered as a benchmark RVE.

The deformation-driven load, $\widehat{u}_{\widehat{M}}=5 \widehat{u}_{\widehat{N}}$, is applied step-wise at the top and in the opposite direction at the bottom, until the maximum lateral top displacement of $\widehat{u}_{\widehat{M}}=0.2 h_{0}$ is reached. A fixed set of material parameters is used. Particularly Young's modulus in the RVE is chosen significantly weaker than that of the macro bulk, $\widehat{E} / 2000=E=p$; lateral contraction is allowed by setting Poisson's ratio to $\widehat{\nu}=\nu=0.3$. The internal length is chosen to be $l=0.1 h_{0}$, while the height of the material layer relative to the height of the macro shear layer is $h_{0} / \widehat{H}_{0}=w_{0} / \widehat{H}_{0}=0.05$.

The column of elements, which represents the periodic shear layer at the macro scale, is shown in Figure 22 for the different RVEs after the last load step, with the corresponding RVE meshes being plotted in Figure 23. For the RVE of Figure 23(d) that represents an infinite homogeneous shear layer, the bulk elements at the macro level are deformed homogeneously as under simple shear, while the RVE itself obeys an S-shape. The latter appears due to the boundary conditions for the micro deformation map at the top and bottom boundaries of the RVE.

Figure 24 shows the load displacement curves at the top of the macro column for the different microstructures. The response to the shear dominated mixed-mode loading caused by the vertically orientated lentil void is the stiffest, while the other two differ for the considered component, i. e. normal or tangential. Contrary to the classical RVE in Reference [8], here the homogeneous RVE does not result in the stiffest response. This can be attributed to the fact that, due to the nearly homogeneous deformation, the additional micro degrees of freedom and thus especially the gradient of the micro deformation map are barely excited and the respective contributions in the potential-energy density (11) do not come into play as strongly as for the heterogeneous specimens.

With this example it is successfully shown that macroscopic boundary value problems involving a material interface can be solved numerically by applying computational homogenization to obtain the response of the latter. 
(a)

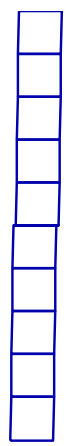

(b)

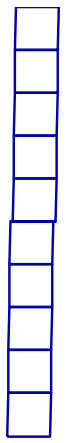

(c)

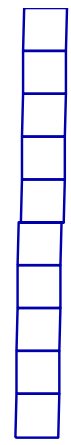

(d)

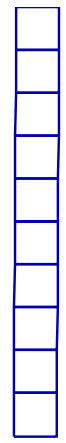

Figure 22. Example 6.3: Spatial macro meshes at $\widehat{u}_{\widehat{M}}=0.2 h_{0}$, for RVE with (a) hole, (b) vertical lentil-shaped void, (c) horizontal lentil-shaped void, (d) homogeneous shear layer.

(a)

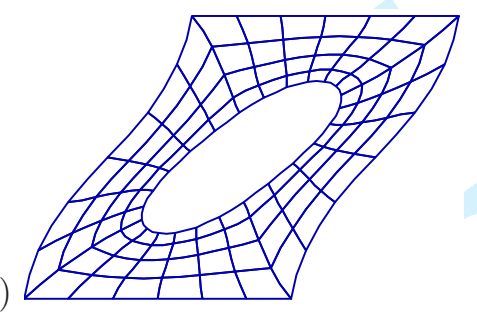

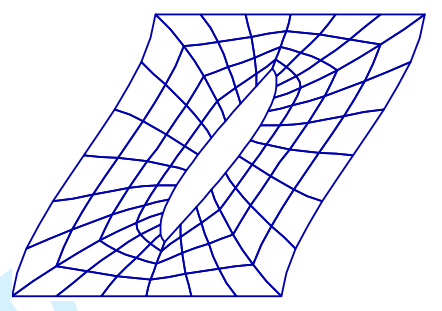

(c)

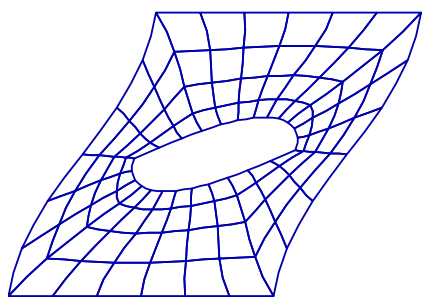

(d)

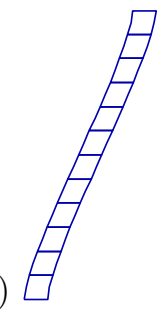

Figure 23. Example 6.3: Spatial RVE meshes with (a) hole, (b) vertical lentil-shaped void, (c) horizontal lentil-shaped void, (d) homogeneous shear layer at $\widehat{u}_{\widehat{M}}=0.02 h_{0}$.
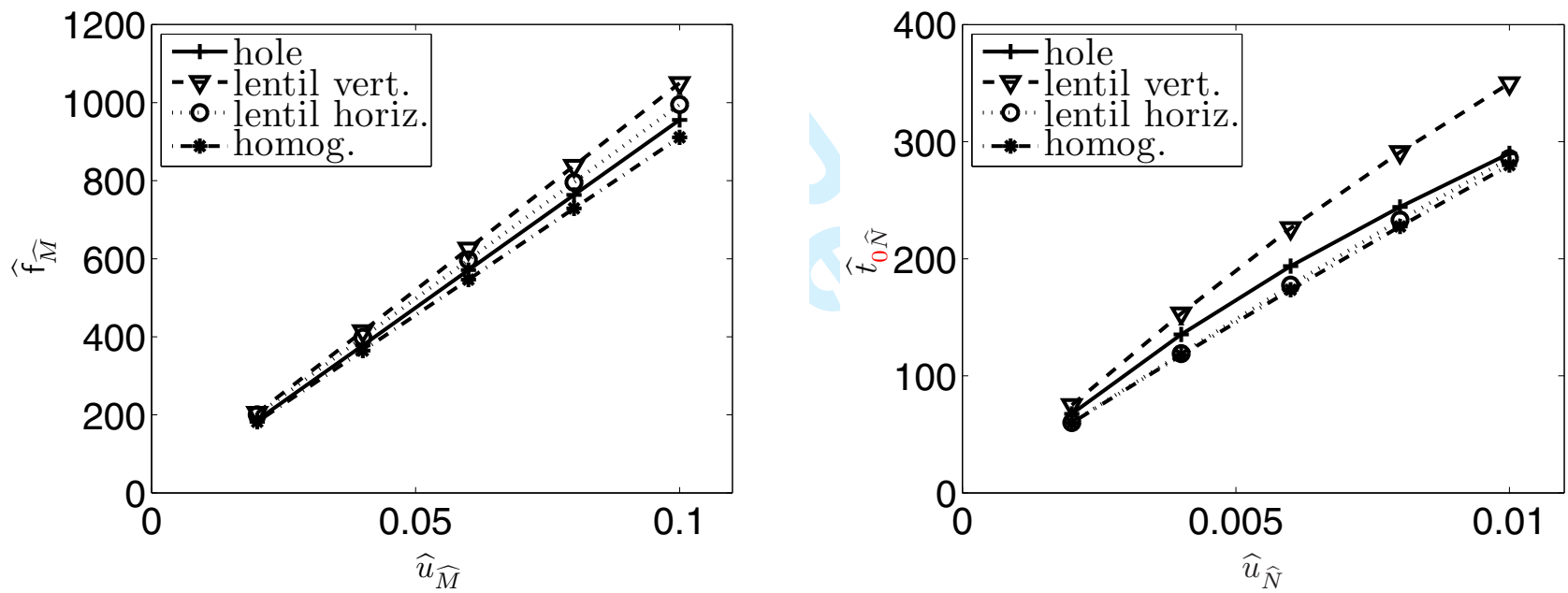

Figure 24. Example 6.3: Macro force-displacement curve at top of the macro shear layer.

\subsection{Material layer within a specimen with a hole}

The forth and last example consists of a square specimen with a circular centred hole at the macro level, comparable to the meso specimen in Sections 6.1 and 6.2, meshed with 72 bulk elements. Additionally at both lateral sides of the hole, a horizontal layer of three interface elements is arranged. The specimen is subject to constant uni-axial tension in vertical direction. At each interface integration point, the underlying micromorphic RVE of Sections 6.1 and 6.2 is evaluated. Once more, the internal-length parameter in the RVE is varied for an otherwise fixed set of material parameters $\left(\widehat{E}=100 E=1 \times 10^{5}, \widehat{\nu}=\nu=0.3\right.$, $\left.p / E=1000=10 \widehat{E}, \widehat{h}_{0}=20 h_{0}\right)$.

In Figure 25, next to the spatial macrostructure, the deformed RVEs at the six interfacial integration points along the material layer are shown for the maximum displacement applied, $\widehat{u}_{\widehat{N}} / \widehat{h}_{0}=0.025$. The particular deformed heights $h_{t}$ of the RVEs qualitatively coincide well with the expected peak of both normal stress and normal strain close to the heterogeneity, the hole. This is also reflected by the normal components of the macro separation, $\llbracket \widehat{\varphi}_{\widehat{N}} \rrbracket$, and the macro traction, $\widehat{t}_{0 \widehat{N}}$, which are plotted versus the 


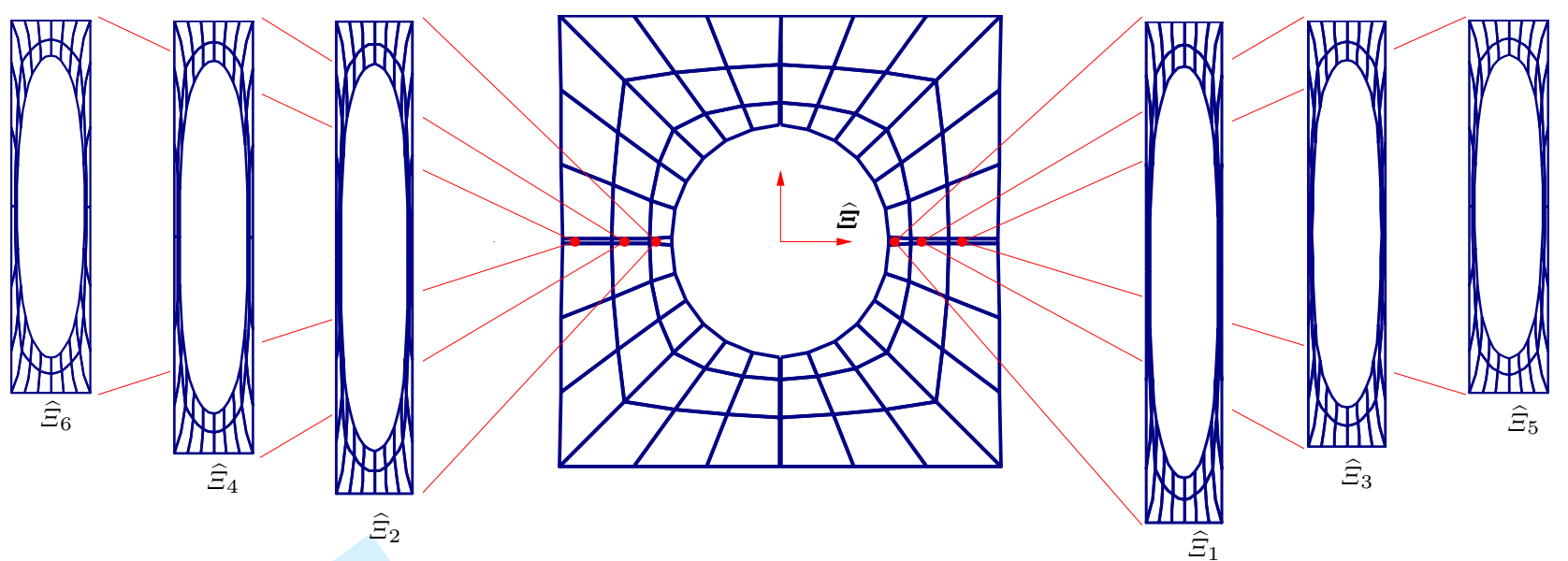

Figure 25. Example 6.4: Spatial macro mesh in the centre with corresponding spatial meso meshes at the macro interface integration points at the macro interface integration points located at coordinates $\widehat{\Xi}_{i}=0.522,0.581,0.638,0.737,0.821,0.952$.
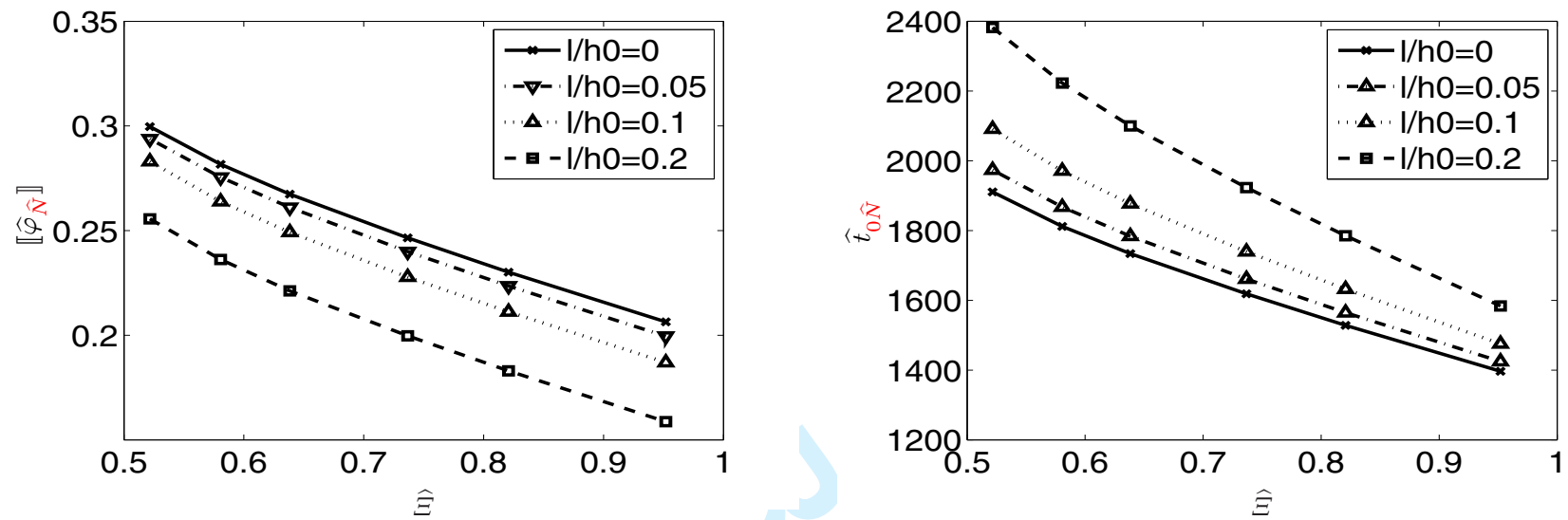

Figure 26. Example 6.4: Separation vs. placement, homogenized traction vs. placement along the interface
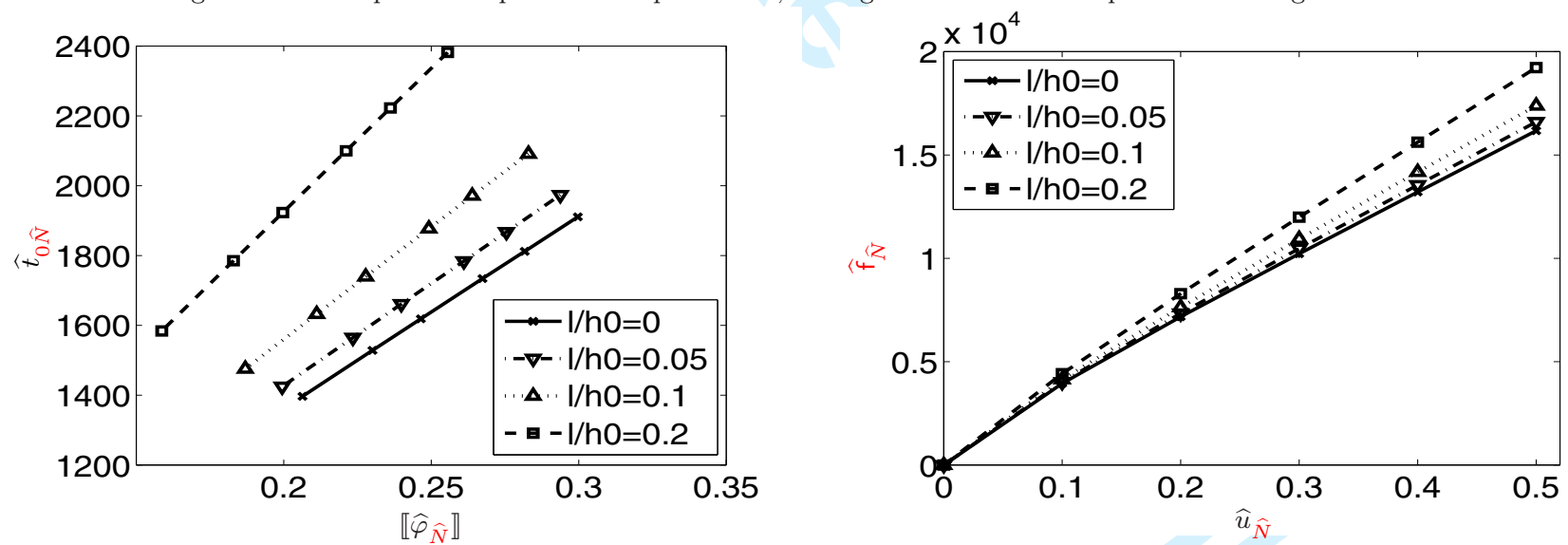

Figure 27. Example 6.4: Traction-separation curve near the hole, load-displacement curve at macro top nodes for different internal-length parameters,

horizontal coordinate of the relative placement, $\widehat{\Xi}$, in Figure 26. This relative horizontal position $\widehat{\Xi}=$ $2 \widehat{X} / \widehat{w}_{0}$ is given relative to the half width of the macro specimen.

While the normal separation $\llbracket \widehat{\varphi}_{\widehat{N}} \rrbracket$ is larger for smaller internal length, the normal traction $\widehat{t}_{0 \widehat{N}}$ increases with internal length. This observation matches well with the stiffer behaviour for larger internal length that the traction-separation curves in Figure 27 display. The macroscopic response is illustrated in the same figure: the prescribed displacement in vertical direction, $u_{\widehat{N}}$, at the top of the macro specimen is plotted versus the total reaction force $\mathrm{f}_{\widehat{N}}$ in vertical direction at this edge. Here an influence of the underlying RVE is observed: once more the specimen with the RVE of the highest internal lengths obeys the stiffest 
macro response.

\section{Conclusion}

\subsection{Summary}

In this contribution, in the framework of finite deformations we have successfully extended the available concepts of theoretical and computational homogenization to material layers with an underlying mesostructure, which is on the one hand heterogeneous (see also Reference [8]) and on the other hand possesses a significant intrinsic microstructure.

We have linked the macro quantities, which are governed by the deformation jump across the material layer, to their corresponding quantities in the micromorphic RVE by homogenization. Thereby the two different types of continua, namely a classical (Boltzmann) continuum on the macro level and a micromorphic continuum on the meso level, are consistently related to each other by means of a homogenization approach. In this context we have formulated boundary conditions which clearly account for the deformation induced by the cohesive interface, while they fulfil the Hill macro-homogeneity condition. Through the incorporation of the micromorphic continuum within the representative volume element, we are now able to account for size-dependence effects that arise due to the underlying microstructure in the material layer, as it can for instance be found in geomaterials, metals, etc.

The proposed computational homogenization framework involves a nested multiscale solution scheme, which comprises both the solution of the nonlinear macro boundary value problem and of the underlying nonlinear RVE boundary value problems. For the latter micromorphic problem, being coupled between the meso and the micro deformation, the formulation of Hirschberger et al. [14] provided the appropriate numerical framework.

\subsection{Outlook}

Besides a hyperelastic representative volume element, it is desirable to involve irreversible phenomena for the meso and micro level in a next step. With such, for instance with plasticity and damage, the presented approach shall yield to the well-known cohesive laws that display softening behaviour. A distinct improvement is particularly expected for mixed-mode loading that now can be treated more accurately under incorporation of the mesostructural response.

By consideration of the micromorphic continuum, besides the macro level including the interface, and the meso level with the representative volume element, a third scale has been intrinsically involved in the model. It can be seen as a challenge for future research to relate the present model representation of the attached microcontinua to actual physical microstructures, such as granular assemblies as for instance treated in References [38,39] and in the framework of homogenization in References [40,41]. Thereby the effects such as inter-granular friction result in non-symmetric homogenized stresses and therefore particularly motivate the use of micromorphic or micropolar continua.

\section{Acknowledgement}

The authors gratefully acknowledge the financial support by the German Science Foundation (DFG) within the International Research Training Group 1131 'Visualization of large and unstructured data sets. Applications in geospatial planning, modeling, and engineering'. 
Appendix A: Admissibility of boundary conditions imposed on the representative volume element with respect to the Hill conditions

To show that the chosen boundary conditions meet the Hill condition (14), the following relations

$$
\langle\boldsymbol{P}\rangle:\langle\delta \boldsymbol{F}\rangle=\langle\boldsymbol{P}:\langle\delta \boldsymbol{F}\rangle\rangle=\langle\langle\boldsymbol{P}\rangle: \delta \boldsymbol{F}\rangle
$$

are used. With these at hand, the Hill condition to be fulfilled is rewritten as:

$$
\begin{aligned}
& h_{0}\langle\boldsymbol{P}: \delta \boldsymbol{F}+\overline{\boldsymbol{P}}: \delta \overline{\boldsymbol{F}}+\overline{\boldsymbol{Q}}: \delta \overline{\boldsymbol{G}}\rangle-h_{0}\langle\boldsymbol{P}:\langle\delta \boldsymbol{F}\rangle\rangle \doteq 0 \\
& h_{0}\langle\boldsymbol{P}: \delta \boldsymbol{F}+\overline{\boldsymbol{P}}: \delta \overline{\boldsymbol{F}}+\overline{\boldsymbol{Q}}: \delta \overline{\boldsymbol{G}}\rangle-h_{0}\langle\langle\boldsymbol{P}\rangle: \delta \boldsymbol{F}\rangle \doteq 0
\end{aligned}
$$

whereby the first equation is utilized for fully prescribed deformation and the latter for constant traction on the RVE boundary.

\section{A.1 Prescribed deformation on the RVE boundary}

To show the admissibility of the deformation boundary conditions of Section 4.2.1, Equation (A2) is verified here:

$$
\begin{aligned}
& h_{0}\langle\boldsymbol{P}: \delta \boldsymbol{F}+\overline{\boldsymbol{P}}: \delta \overline{\boldsymbol{F}}+\overline{\boldsymbol{Q}}: \delta \overline{\boldsymbol{G}}\rangle-h_{0}\langle\boldsymbol{P}:\langle\delta \boldsymbol{F}\rangle\rangle \\
= & \frac{1}{w_{0}} \int_{\partial \mathcal{B}_{0}} \delta \boldsymbol{\varphi} \cdot \boldsymbol{P} \cdot \boldsymbol{N}-\langle\delta \boldsymbol{F}\rangle:[\boldsymbol{P} \cdot \boldsymbol{N} \otimes \boldsymbol{X}]+\delta \overline{\boldsymbol{F}}: \overline{\boldsymbol{Q}} \cdot \boldsymbol{N} \mathrm{d} A=\frac{1}{w_{0}} \int_{\partial \mathcal{B}_{0}}[\delta \boldsymbol{\varphi}-\langle\delta \boldsymbol{F}\rangle \cdot \boldsymbol{X}] \cdot \boldsymbol{t}_{0}+\delta \overline{\boldsymbol{F}} \cdot \overline{\boldsymbol{t}}_{0} \mathrm{~d} A \doteq 0
\end{aligned}
$$

This term identically equals zero if the deformation boundary condition (16) is applied.

\section{A.2 Constant traction on the RVE boundary}

In view of the traction boundary condition of Section 4.2.2, identity (A3) is modified in the following steps:

$$
\begin{aligned}
& h_{0}\langle\boldsymbol{P}: \delta \boldsymbol{F}+\overline{\boldsymbol{P}}: \delta \overline{\boldsymbol{F}}+\overline{\boldsymbol{Q}}: \delta \overline{\boldsymbol{G}}\rangle-h_{0}\langle\langle\boldsymbol{P}\rangle: \delta \boldsymbol{F}\rangle \\
= & \frac{1}{w_{0}} \int_{\partial \mathcal{B}_{0}} \delta \boldsymbol{\varphi} \cdot[\boldsymbol{P}-\langle\boldsymbol{P}\rangle] \cdot \boldsymbol{N}+\delta \overline{\boldsymbol{F}}: \overline{\boldsymbol{Q}} \cdot \boldsymbol{N} \mathrm{d} A=\frac{1}{w_{0}} \int_{\partial \mathcal{B}_{0}} \delta \boldsymbol{\varphi} \cdot\left[\boldsymbol{t}_{0}-\langle\boldsymbol{P}\rangle \cdot \boldsymbol{N}\right]+\delta \overline{\boldsymbol{F}}: \overline{\boldsymbol{t}}_{0} \mathrm{~d} A \doteq 0 .
\end{aligned}
$$

From this result, it can easily be seen that for the choice of traction boundary conditions (18) with the Piola type stress equivalence (13), this requirement is fulfilled identically .

\section{A.3 Periodic deformation and antiperiodic traction on the RVE boundary}

From the derivation (A4) for prescribed deformations, it is straightforward to recognize that periodicity in deformations and antiperiodicity in tractions of Section 4.2.3 fulfil the condition (A2). Particularly, $\langle\delta \boldsymbol{F}\rangle \cdot \boldsymbol{X}$ is a priori periodic for the macro deformation affinely imposed on the RVE. With this, the fluctuation term $[\delta \boldsymbol{\varphi}-\langle\delta \boldsymbol{F}\rangle \cdot \boldsymbol{X}]$ is periodic and together with antiperiodic tractions, the integral over opposite boundaries vanishes. The same holds for the micro deformation map, which must go along with antiperiodic double tractions. A detailed proof is elaborated in Reference [15]. 


\section{A.4 Hybrid boundary conditions on the RVE}

For the hybrid choice of boundary conditions presented in Section 4.2.4, the derivation for the top and bottom boundary is already given by Equation (A4). The validity of the periodicity for the opposite left right edges can directly be adopted from the fully periodic case, Section A.3.

\section{Appendix B: Tangent operator from computational homogenization}

The tangent operator (27) is derived from the increment of the traction (26) with the reaction forces in the coupled system (25) and the prescribed deformation (21) and (22) at the top and bottom nodes:

$$
\begin{aligned}
\Delta \widehat{\boldsymbol{t}}_{0} & =\frac{1}{V_{0}} \sum_{I} \Delta \mathbf{f}_{0 I} \otimes \boldsymbol{X}_{I} \cdot \widehat{\boldsymbol{N}}=\frac{1}{V_{0}} \sum_{I} \sum_{K, L}\left[\left[\mathbf{K}_{I K}^{\varphi \varphi} \cdot \Delta \boldsymbol{\varphi}_{K}+\mathbf{K}_{I L}^{\varphi \bar{F}} \cdot \Delta \overline{\boldsymbol{F}}_{L}\right] \otimes \boldsymbol{X}_{I}\right] \cdot \widehat{\boldsymbol{N}} \\
& =\frac{1}{V_{0}} \sum_{I} \sum_{K, L}\left[\left[\mathbf{K}_{I K}^{\varphi \varphi} \cdot\left[\frac{1}{h_{0}} \llbracket \Delta \widehat{\boldsymbol{\varphi}} \rrbracket \otimes \widehat{\boldsymbol{N}}\right] \cdot \boldsymbol{X}_{K}+\mathbf{K}_{I, L}^{\varphi \bar{F}} \cdot \Delta \overline{\boldsymbol{F}}_{L}\right] \otimes \boldsymbol{X}_{I}\right] \cdot \widehat{\boldsymbol{N}} \\
& =\left[\frac{1}{h_{0}^{2} w_{0}}[\widehat{\boldsymbol{N}} \otimes \widehat{\boldsymbol{N}}]: \sum_{I} \sum_{K} \mathbf{K}_{I K}^{\varphi \varphi}:\left[\boldsymbol{X}_{K} \otimes \boldsymbol{X}_{I}\right] \cdot\right] \llbracket \Delta \widehat{\boldsymbol{\varphi}} \rrbracket
\end{aligned}
$$

Herein the term in the outer brackets is identified as the macro tangent operator, which relates the current increment of the macro deformation jump to the current traction increment. Thereby the trivial identity $\Delta \boldsymbol{I}=\mathbf{0}$ has been used and, based on the assumption (22), $\Delta \overline{\boldsymbol{F}}=\mathbf{0}$ encounters the coupled contribution of $\mathbf{K}_{I L}^{\varphi \bar{F}}$ to vanish.

\section{References}

[1] X.-P. Xu and A. Needleman. Void nucleation by inclusion debonding in a crystal matrix. Modelling Simul. Mater. Sci. Eng., $1: 111-132,1993$.

[2] M. J. van den Bosch, P. J. G. Schreurs, and M. G. D. Geers. An improved description of the exponential Xu and Needleman cohesive zone law for mixed-mode decohesion. Eng. Fract. Mech., 73:1220-1234, 2006.

[3] E. C. Aifantis. On the microstructural origin of certain inelastic models. J. Engng. Mater. Technol., 106:326-330, 1984.

[4] N. A. Fleck and J. W. Hutchinson. A phenomenological theory for strain gradient effects in plasticity. J. Mech. Phys. Solid., 41:1825-1857, 1993.

[5] A. Nemat-Nasser and M. Hori. Micromechanics: Overall Properties of Heterogeneous Materials. North-Holland Elsevier, 2nd revised edition, 1999.

[6] R. Hill. Elastic properties of reinforced solids: Some theoretical principles. J. Mech. Phys. Solid., 11:357-372, 1963.

[7] R. Hill. On constitutive macro-variables for heterogeneous solids at finite strain. Proc. Roy. Soc. Lond. A, 326:131-147, 1972.

[8] C. B. Hirschberger, S. Ricker, P. Steinmann, and N. Sukumar. Multiscale modelling of heterogeneous material layers. submitted for publication, 2008.

[9] A. C. Eringen. Mechanics of micromorphic materials. In H. Görtler, editor, Proc. 11th Int. Congress of Appl. Mech., pages 131-138, New York, 1964. Springer-Verlag.

[10] A. C. Eringen. Mechanics of micromorphic continua. In E. Kröner, editor, Mechanics of Generalized Continua, Freudenstadt/Stuttgart, 1968. IUTAM Symposium, Springer.

[11] A. C. Eringen. Balance laws of micromorphic mechanics. Int. J. Eng. Sci., 8:818-828, 1970.

[12] N. Kirchner and P. Steinmann. A unifying treatise on variational principles for gradient and micro-morphic continua. Phil. Mag., $85: 3875-3895,2005$.

[13] C. B. Hirschberger, E. Kuhl, and P. Steinmann. Computational modelling of micromorphic continua - theory, numerics, and visualisation challenges. In H. Hagen, A. Kerren, and P. Dannenmann, editors, Visualization of large and unstructured data sets, volume S-4 of GI-Edition Lecture Notes in Informatics (LNI), pages 155-164, 2006.

[14] C. B. Hirschberger, E. Kuhl, and P. Steinmann. On deformational and configurational mechanics of micromorphic hyperelasticity theory and computation. Comput. Methods Appl. Mech. Engrg., 196:4027-4044, 2007.

[15] C. B. Hirschberger. A Treatise on Micromorphic Continua. Theory, Homogenization, Computation. PhD thesis, University of Kaiserslautern, 2008. ISSN 1610-4641, ISBN 978-3-939432-80-7.

[16] C. Miehe, J. Schröder, and J. Schotte. Computational homogenization analysis in finite plasticity. Simulation of texture development in polycrystalline materials. Comput. Methods Appl. Mech. Engrg., 171:387-418, 1999.

[17] F. Feyel and J.-L. Chaboche. FE ${ }^{2}$ multiscale approach for modelling the elastoviscoplastic behaviour of long fibre SiC/Ti composites materials. Comput. Methods Appl. Mech. Engrg., 183:309-330, 2000.

[18] V. G. Kouznetsova, W. A. M. Brekelmans, and F. P. T. Baaijens. An approach to micro-macro modeling of heterogeneous materials. Comput. Mech., 27:37-48, 2001.

[19] C. Miehe, J. Schröder, and C. Bayreuther. On the homogenization analysis of composite materials based on discretized fluctuations on the micro-structure. Comput. Mater. Sci., 155:1-16, 2002. 
[20] C. Miehe and A. Koch. Computational micro-to-macro transitions of discretized microstructures undergoing small strains. Arch. Appl. Mech., 72:300-317, 2002.

[21] V. G. Kouznetsova, M. G. D. Geers, and W. A. M. Brekelmans. Multi-scale constitutive modelling of heterogeneous materials with a gradient-enhanced computational homogenization scheme. Int. J. Numer. Meth. Engng, 54:1235-1260, 2002.

[22] V. G. Kouznetsova, M. G. D. Geers, and W. A. M. Brekelmans. Multi-scale second-order computational homogenization of multiphase materials: a nested finite element solution strategy. Comput. Methods Appl. Mech. Engrg., 193:5525-5550, 2004.

[23] R. Larsson and Y. Zhang. Homogenization of microsystem interconnects based on micropolar theory and discontinuous kinematics. J. Mech. Phys. Solid., 55:819-841, 2007.

[24] M. G. D. Geers, E. W. C. Coenen, and V. G. Kouznetsova. Multi-scale computational homogenization of structured thin sheets. Modelling Simul. Mater. Sci. Eng., 15:393-404, 2007.

[25] G. Beer. An isoparametric joint/interface element for finite element analysis. Int. J. Numer. Meth. Engng, 21:585-600, 1985.

[26] J. C. J. Schellekens and R. de Borst. A non-linear finite element approach for the analysis of mode-I free edge delamination in composites. Int. J. Solid Struct., 30:1239-1253, 1993.

[27] J. Utzinger, M. Bos, M. Floeck, A. Menzel, E. Kuhl, R. Renz, K. Friedrich, A.K. Schlarb, and P. Steinmann. Computational modelling of thermal impact welded PEEK/steel single lap tensile specimens. Comput. Mater. Sci., 41(3):287-296, 2008.

[28] J. Utzinger, A. Menzel, P. Steinmann, and A. Benallal. Aspects of bifurcation in an isotropic elastic continuum with orthotropic inelastic interface. Eur. J. Mech. Solid., 27:532-547, 2008.

[29] R. Larsson, K. Runesson, and N. S. Ottosen. Discontinuous displacement approximation for capturing plastic localization. Int. J. Numer. Meth. Engng, 36:2087-2105, 1993.

[30] C. Miehe and J. Schröder. Post-critical discontinuous localization analysis of small-strain softening elastoplastic solids. Arch. Appl. Mech., 64:267-285, 1994.

[31] P. Steinmann. On localisation analysis in multisurface hyperelasto-plasticity. J. Mech. Phys. Solid., 44:1691-1713, 1996.

[32] R. Larsson, P. Steinmann, and K. Runesson. Finite element embedded localization band for finite strain plasticity based on a regularized strong discontinuity. Mech. of Cohes.-Frict. Mater., 4:171-194, 1998.

[33] P. Steinmann and P. Betsch. A localization capturing FE-interface based on regularized strong discontinuities at large inelastic strains. Int. J. Solid Struct., 37:4061-4082, 2000.

[34] J. Mergheim, E. Kuhl, and P. Steinmann. A hybrid discontinuous Galerkin/interface method for the computational modelling of failure. Commun. Numer. Meth. Engng, 20:511-519, 2004.

[35] C. Miehe. Computational micro-to-macro transitions discretized micro-structures of heterogeneous materials at finite strains based on the minimization of averaged incremental energy. Comput. Methods Appl. Mech. Engrg., 192:559-591, 2003.

[36] F. Costanzo, G. L. Gray, and P. C. Andia. On the definitions of effective stress and deformation gradient for use in MD: Hill's macro-homogeneity and the virial theorem. Int. J. Numer. Meth. Engng, 43:533-555, 2005.

[37] V. G. Kouznetsova. Computational Homogenization for the Multiscale Analysis of Multi-Phase Materials. PhD thesis, Eindhoven University of Technology, 2002.

[38] H. A. Meier, E. Kuhl, and P. Steinmann. A note on the generation of periodic granular microstructures based on grain size distributions. Int. J. Numer. Anal. Meth. Geomech., 32(5):509-522, 2008.

[39] H. A. Meier, P. Steinmann, and E. Kuhl. Towards multiscale computation of confined granular media - contact forces, stresses and tangent operators. Technische Mechanik, 28:32-42, 2008.

[40] W. Ehlers, E. Ramm, S. Diebels, and G. A. D'Addetta. From particle ensembles to Cosserat continua: homogenization of contact forces towards stresses and couple stresses. Int. J. Solid Struct., 40:6681-6702, 2003.

[41] C. Miehe and J. Dettmar. A framework for micro-to-macro transitions in periodic particle aggregates of granular materials. Comput. Methods Appl. Mech. Engrg., 193:225-256, 2004. 


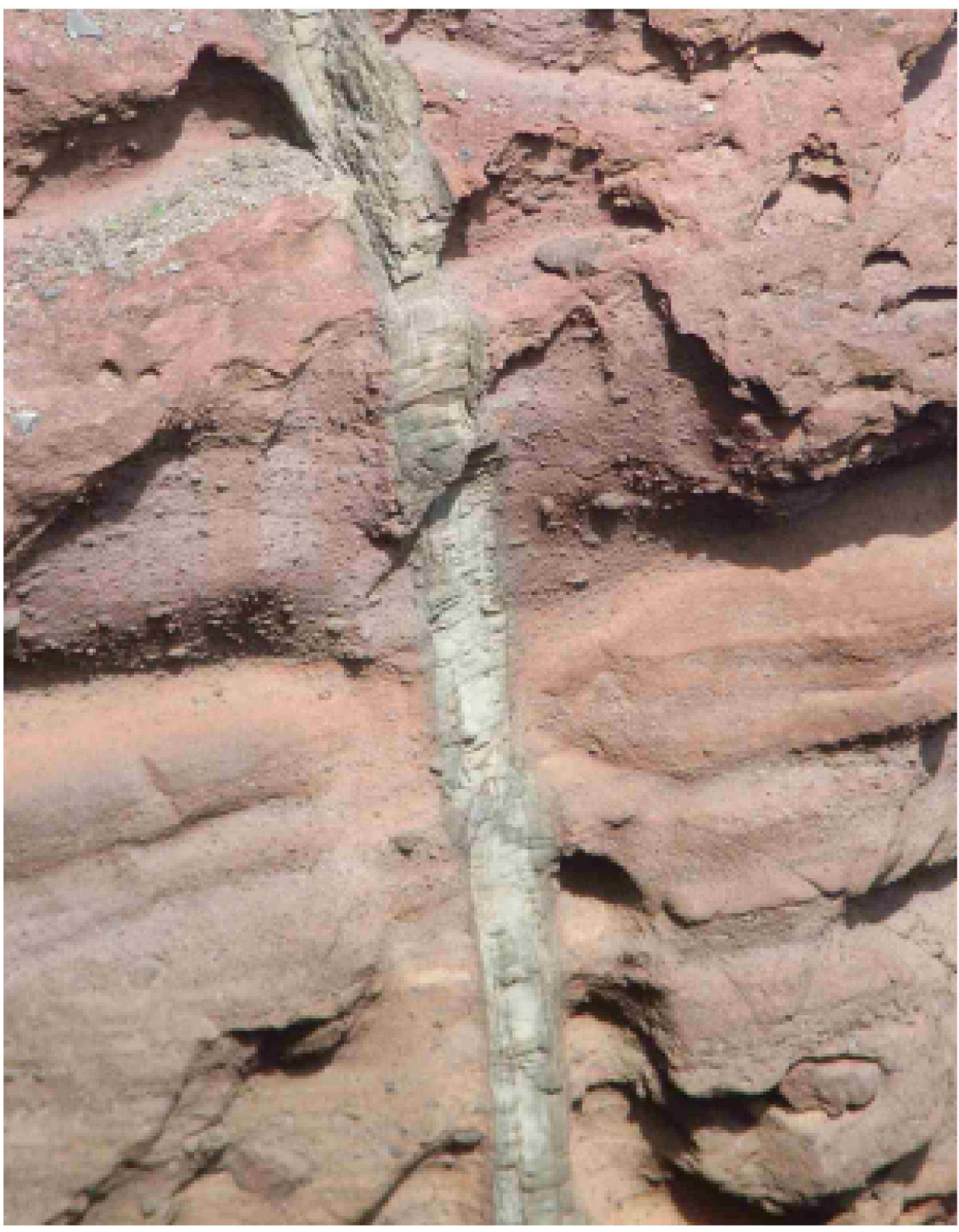

Example for a material layer within a bulk material of different properties $106 \times 135 \mathrm{~mm}(600 \times 600 \mathrm{DPI})$ 

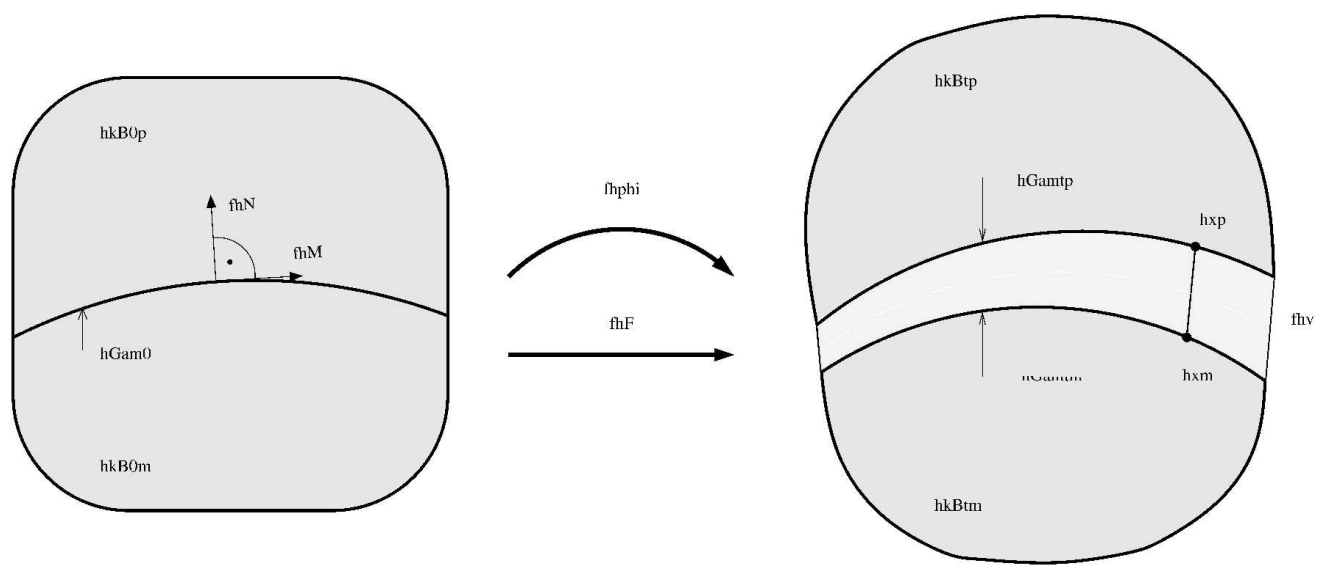

Interface geometry and deformation maps from the material configuration to the spatial configuration $300 \times 127 \mathrm{~mm}(600 \times 600 \mathrm{DPI})$ 


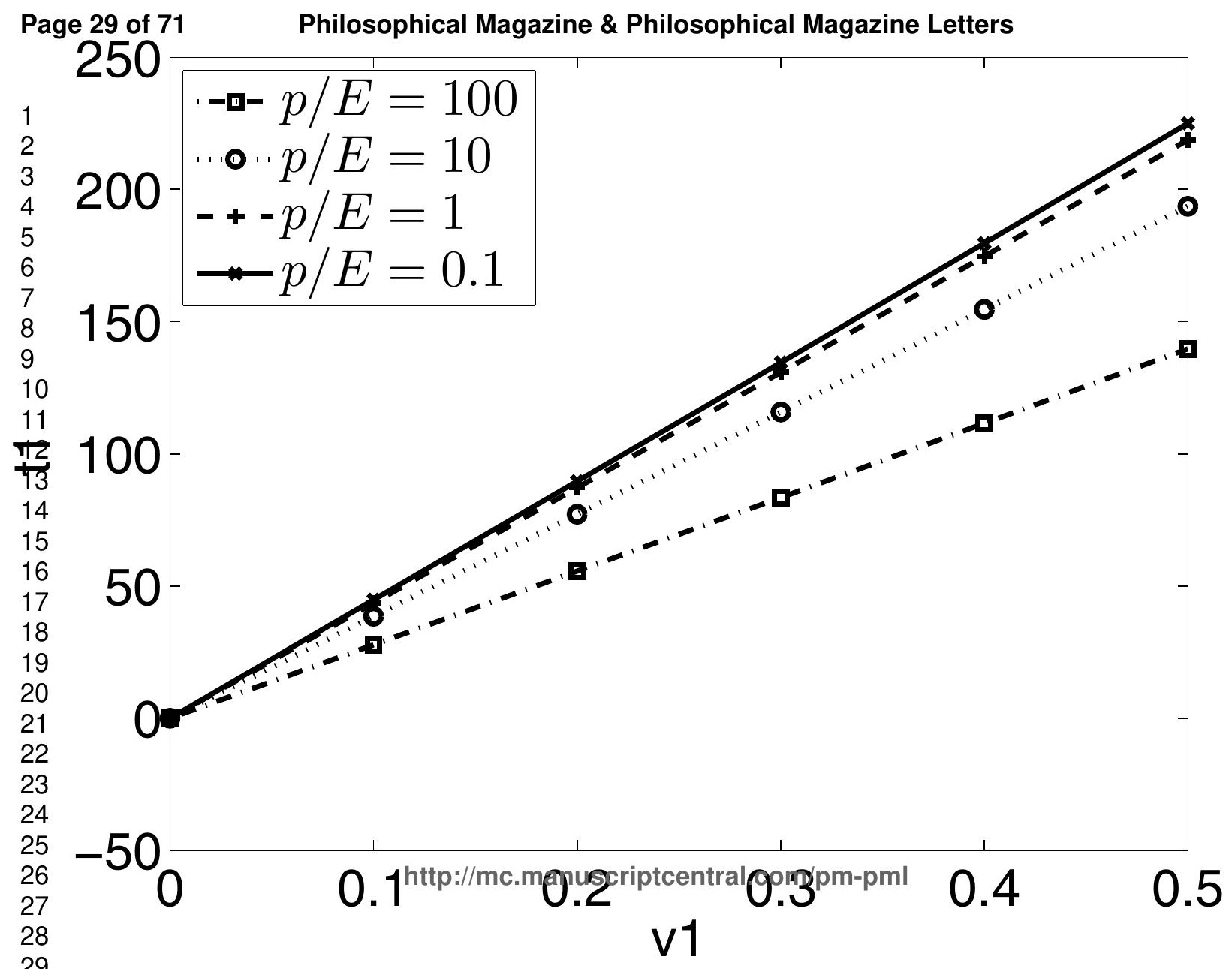




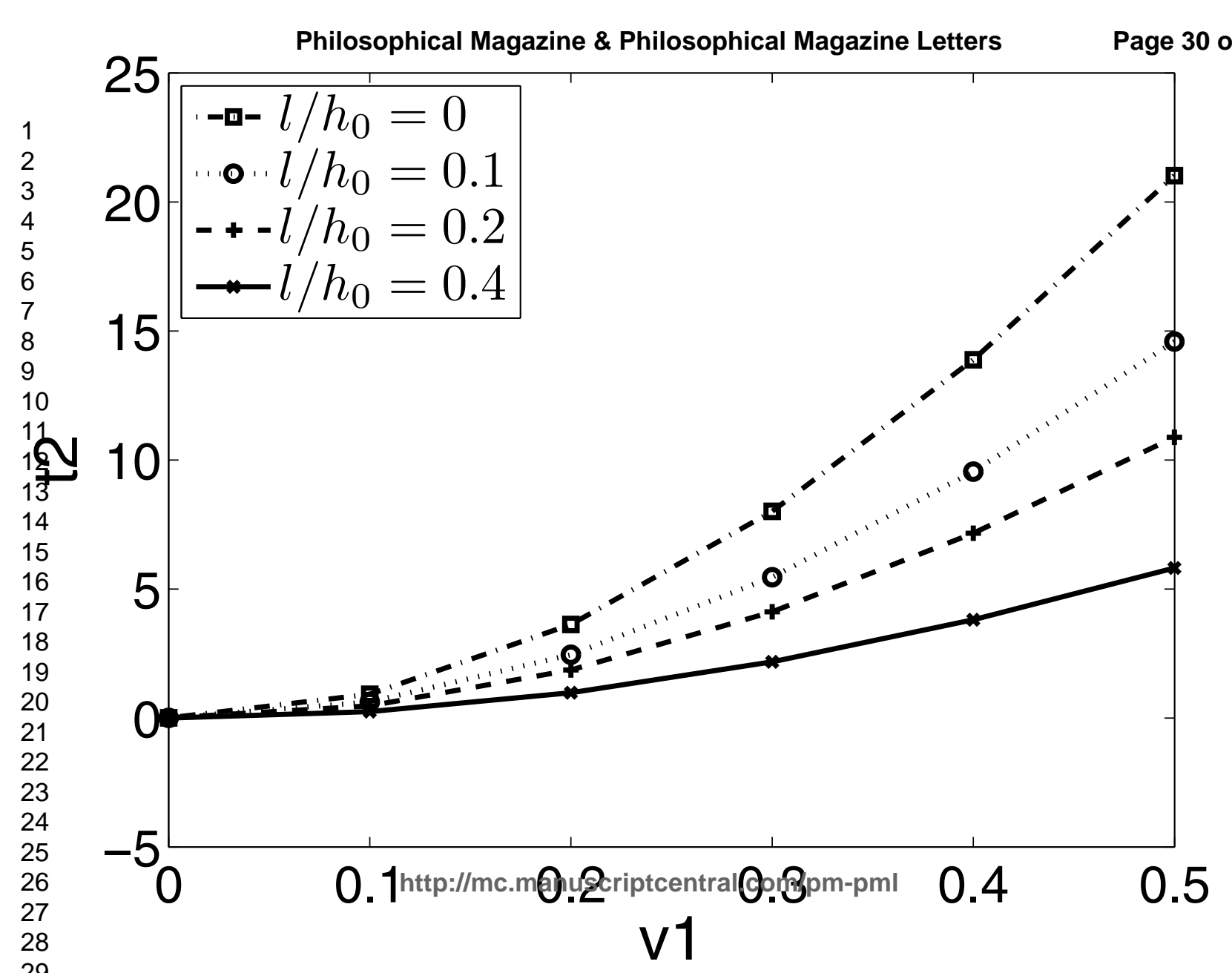




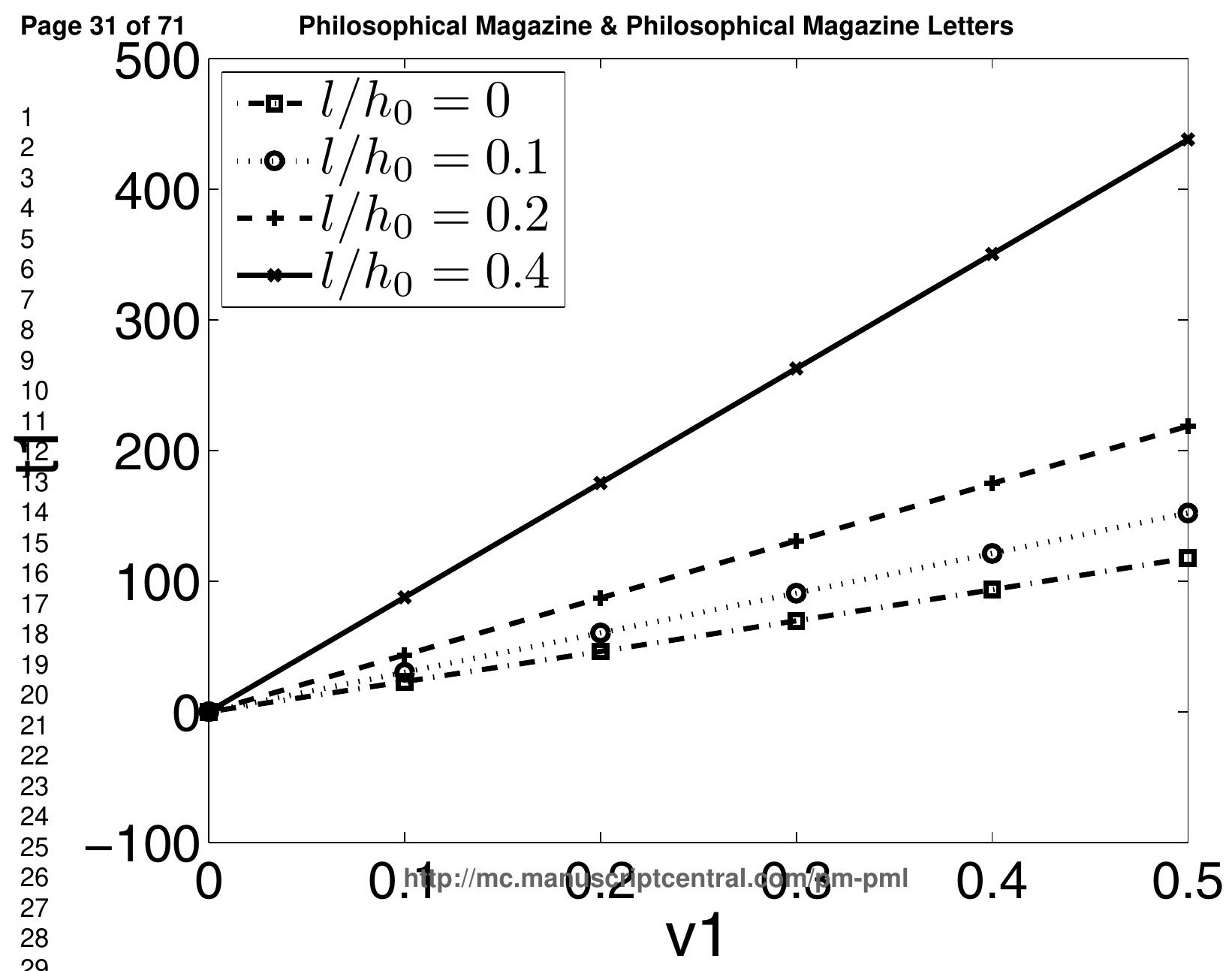




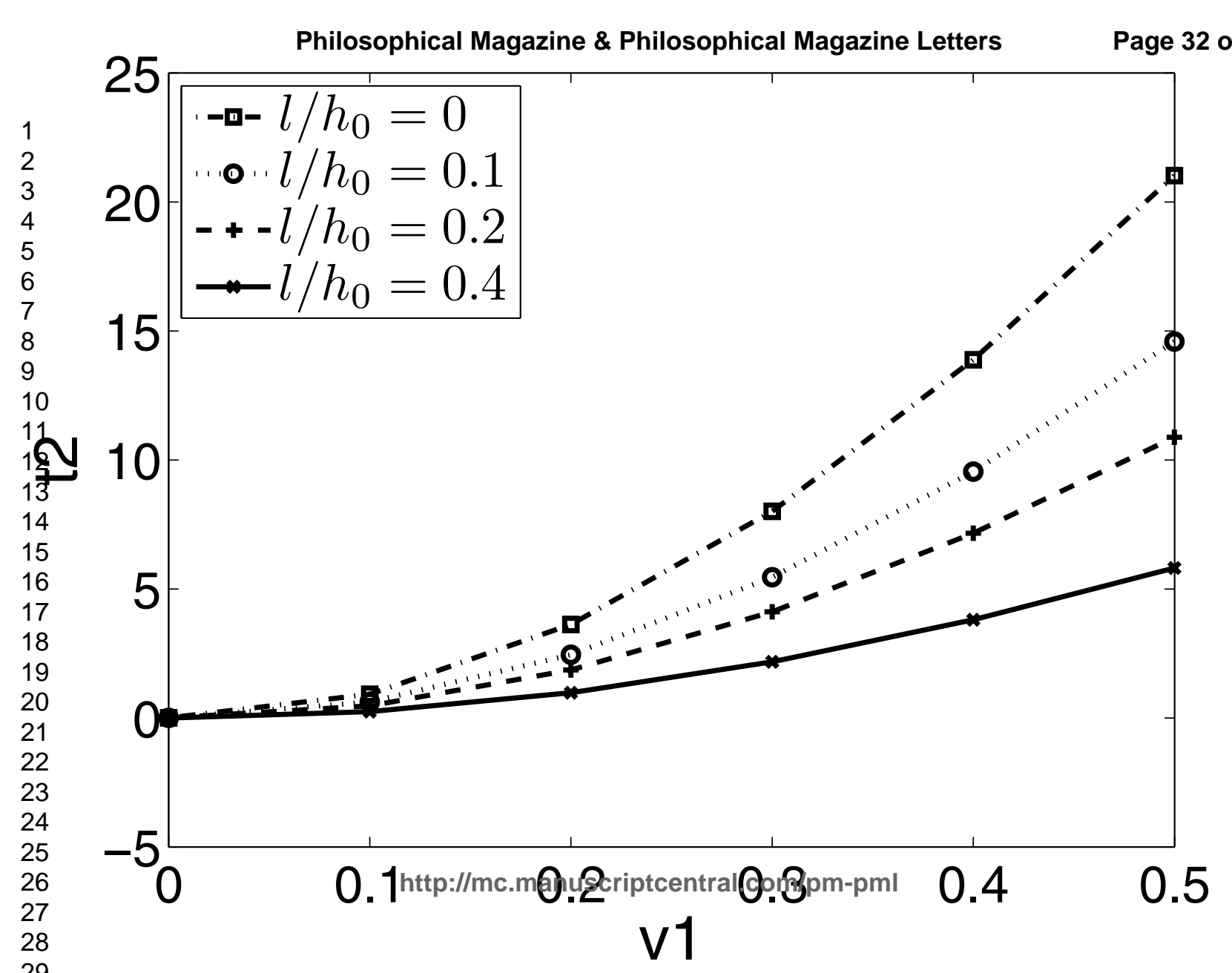




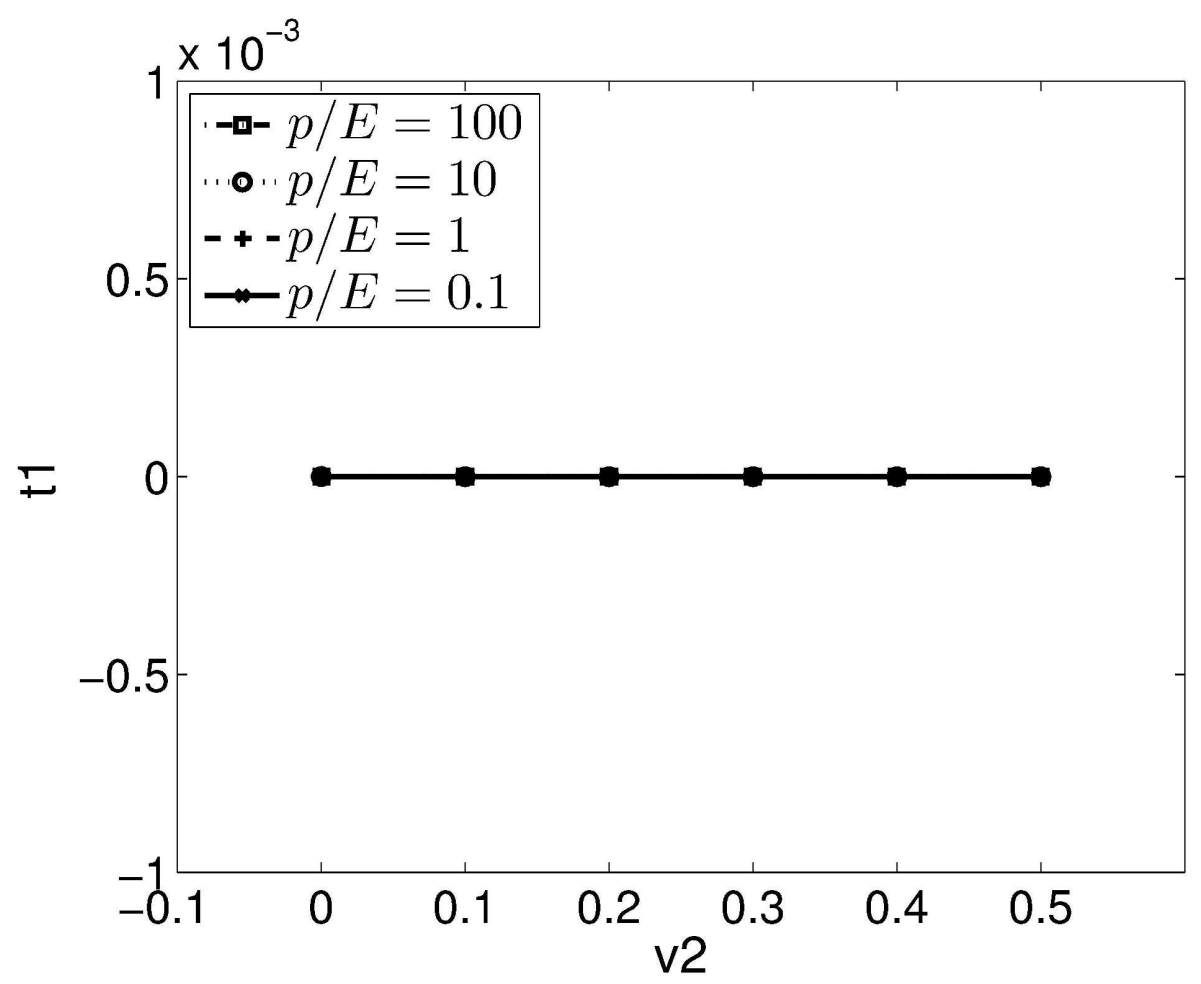

$177 \times 133 \mathrm{~mm}(600 \times 600 \mathrm{DPI})$ 
Philosophical Magazine \& Philosophical Magazine Letters

Page 34 of 71

1
2
3
4
5
6
7
8
9
10
11
12
13
14
15
16
17
18
19
20
21
22
23
24
25
26
27
28
29
30
31
32
33
34
35
36
37
38
39
40
41
42
43
44
45
46
47
48
49
50
51
52
53
54
55
56
57
58
59
60

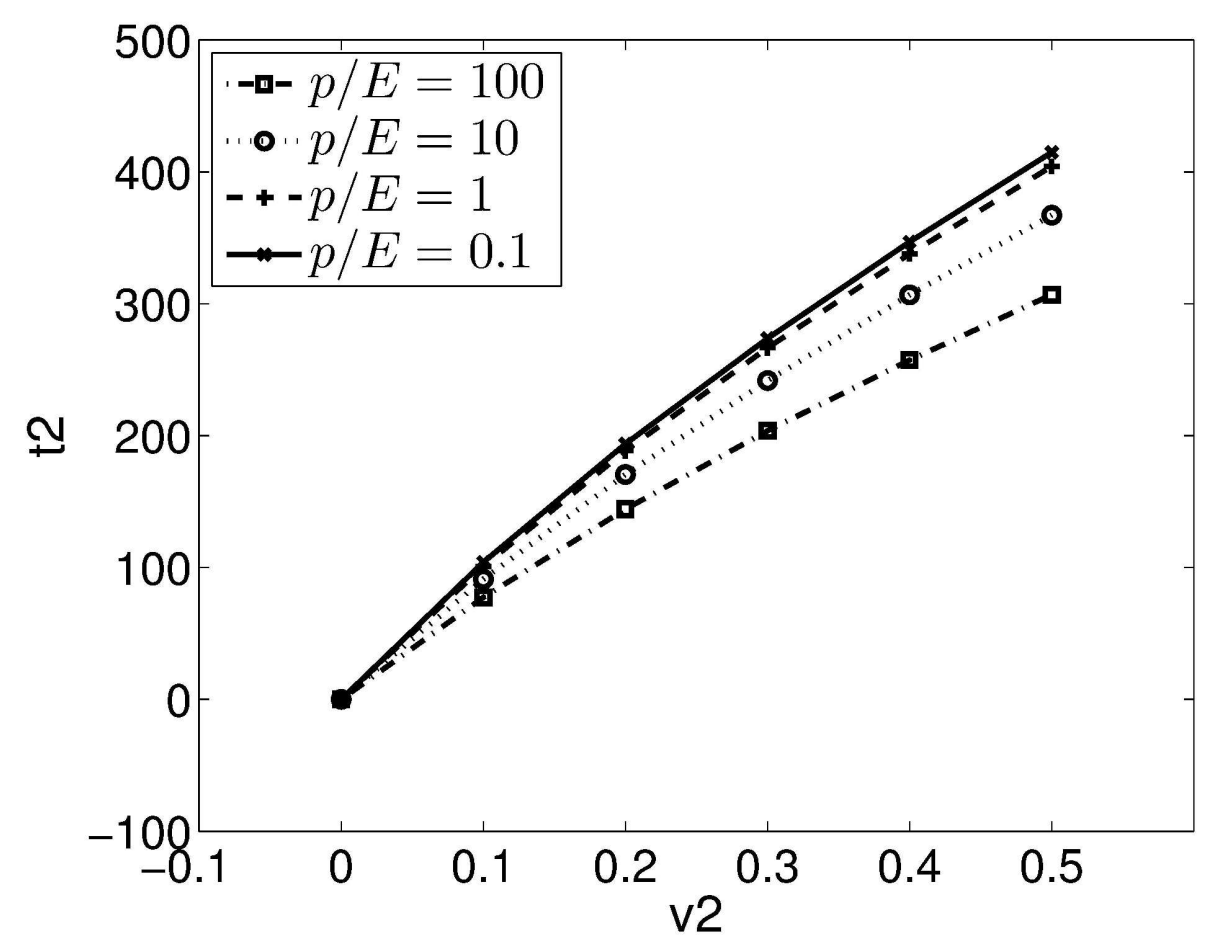

$177 \times 133 \mathrm{~mm}(600 \times 600 \mathrm{DPI})$

http://mc.manuscriptcentral.com/pm-pml 


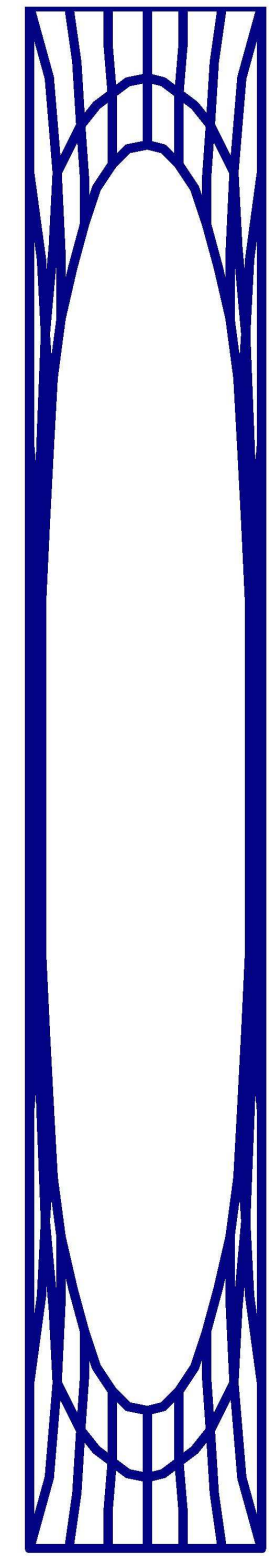

$22 \times 125 \mathrm{~mm}(600 \times 600 \mathrm{DPI})$ 


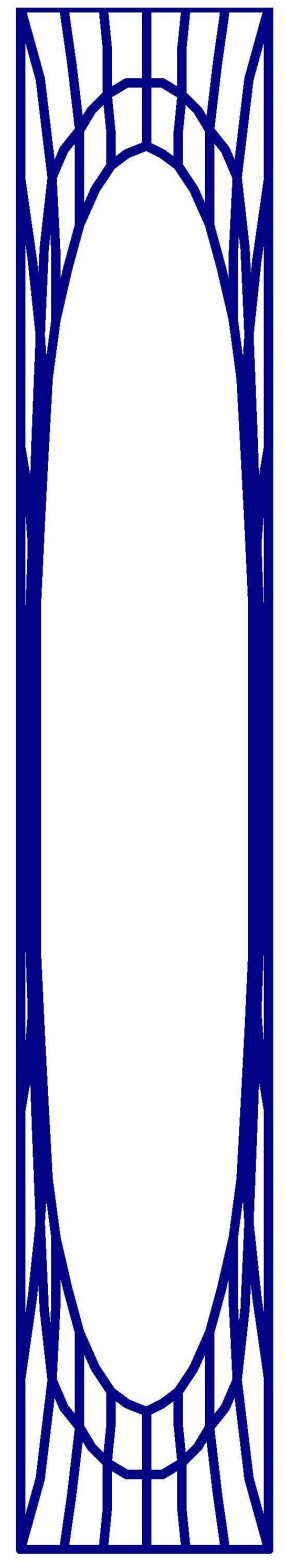

$23 \times 125 \mathrm{~mm}(600 \times 600 \mathrm{DPI})$ 


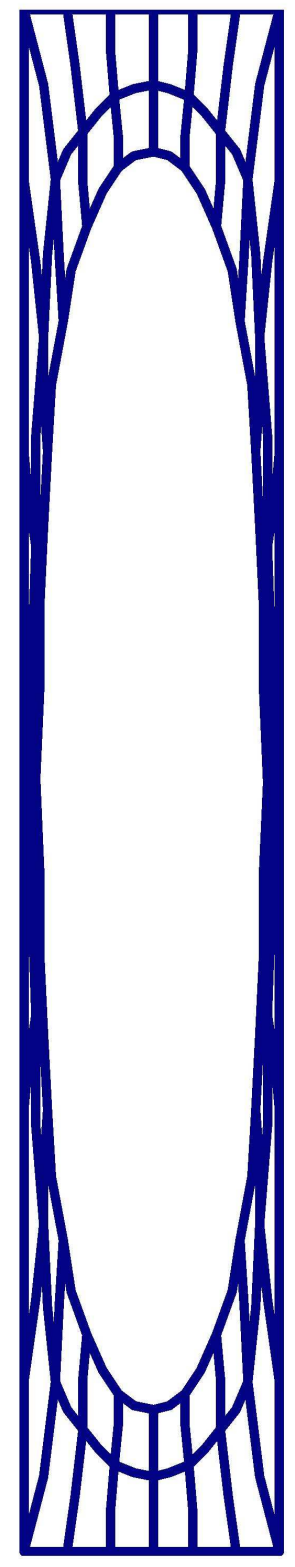

$24 \times 125 \mathrm{~mm}(600 \times 600 \mathrm{DPI})$ 


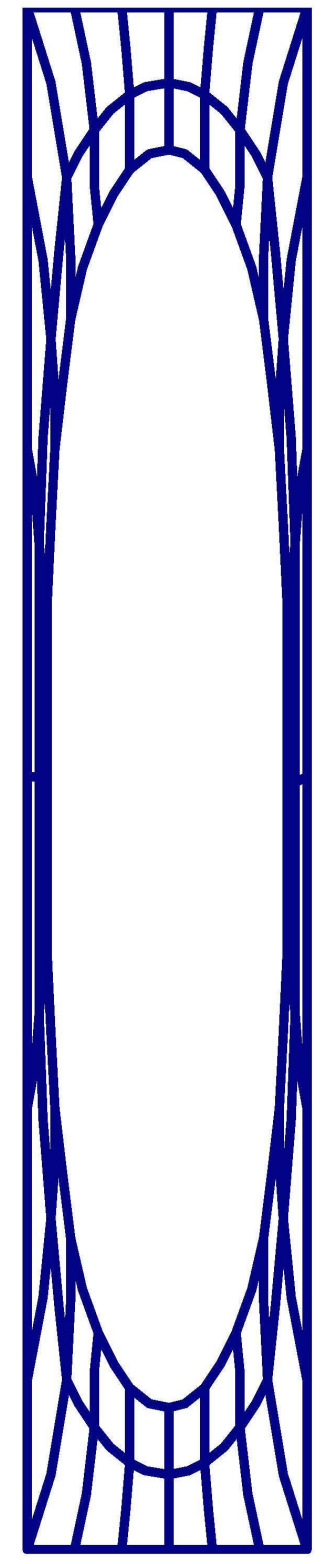

$26 \times 125 \mathrm{~mm}(600 \times 600 \mathrm{DPI})$ 


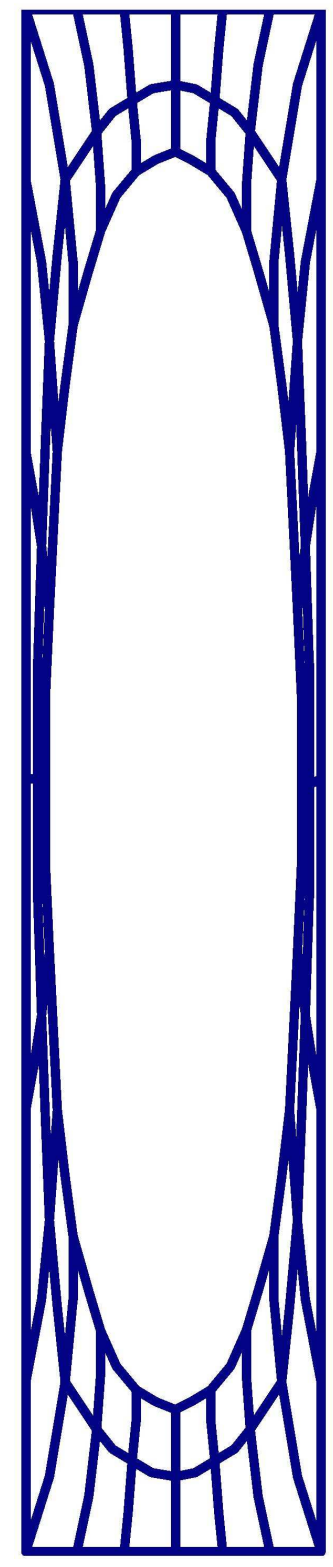

$27 \times 125 \mathrm{~mm}(600 \times 600 \mathrm{DPI})$ 


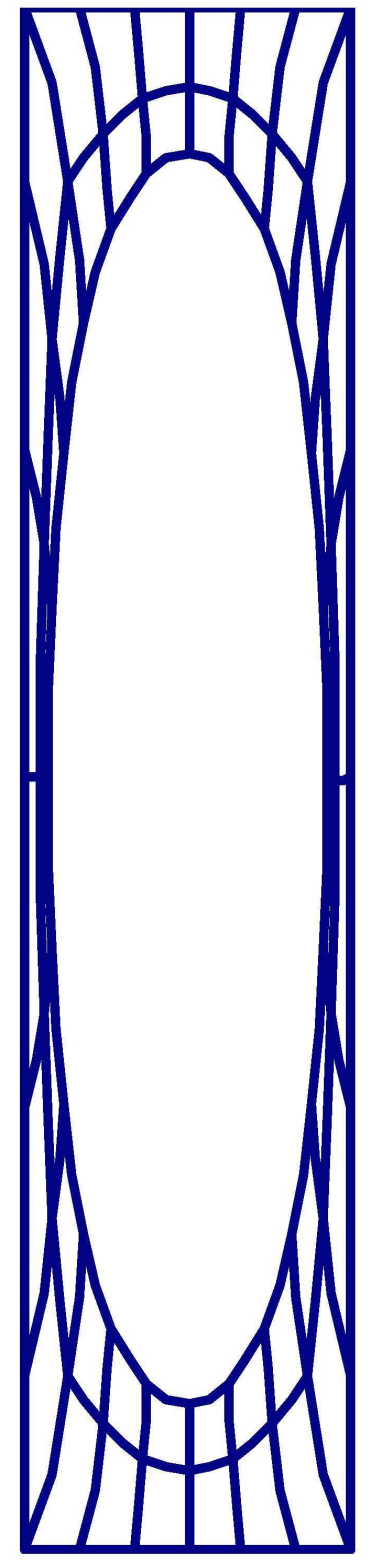

$29 \times 125 \mathrm{~mm}(600 \times 600 \mathrm{DPI})$ 


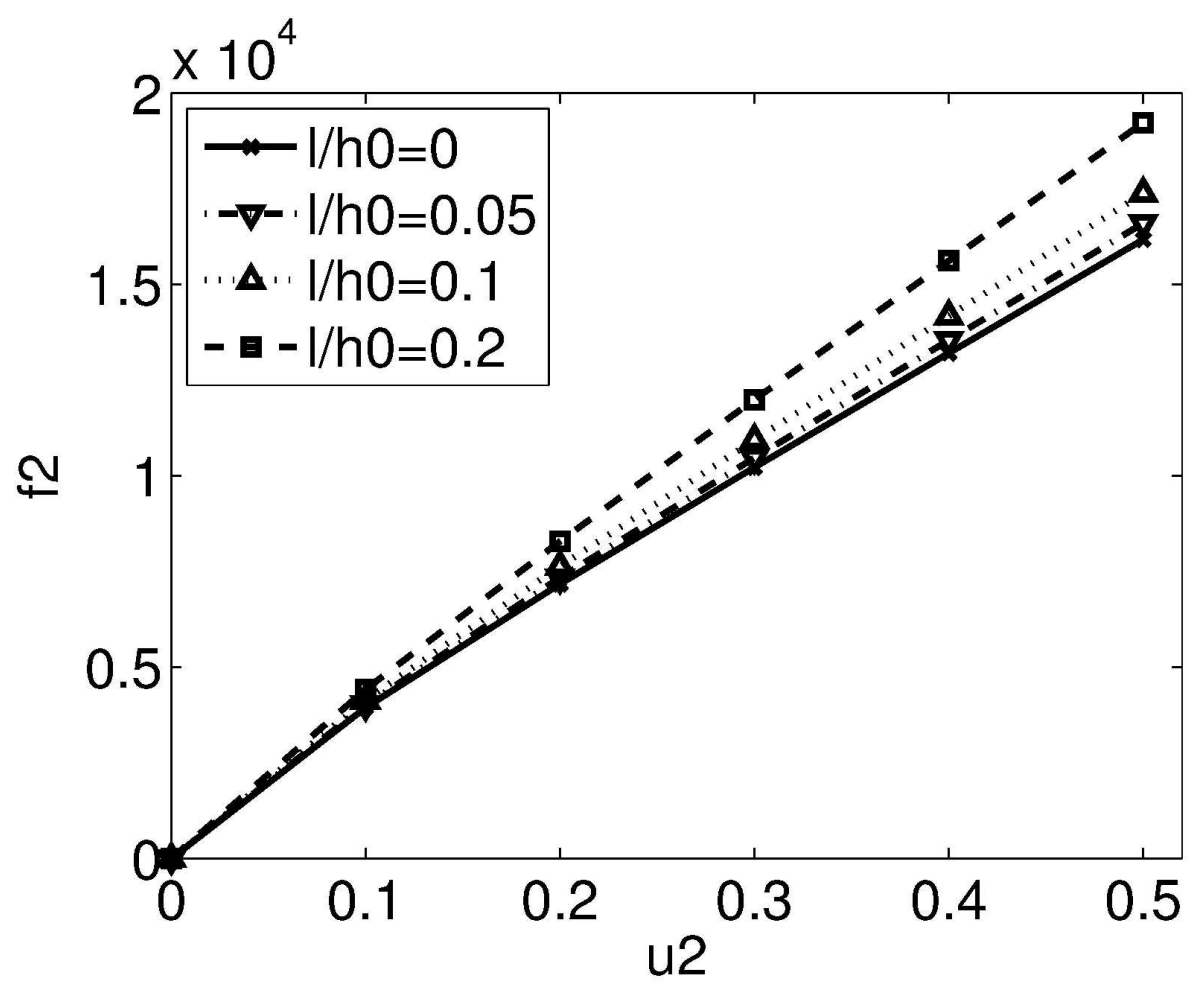

$148 \times 111 \mathrm{~mm}(600 \times 600 \mathrm{DPI})$ 


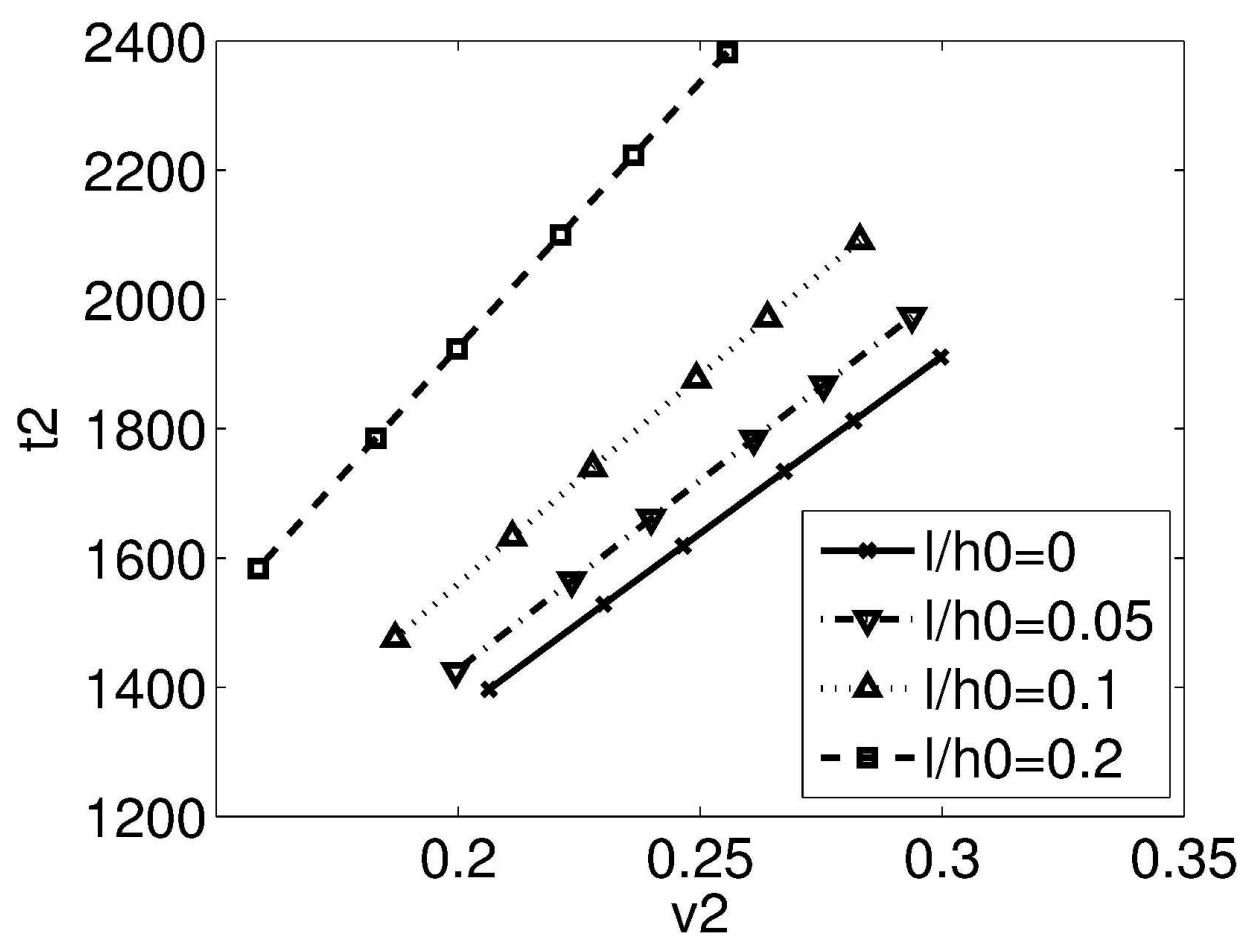

$148 \times 111 \mathrm{~mm}(600 \times 600 \mathrm{DPI})$ 


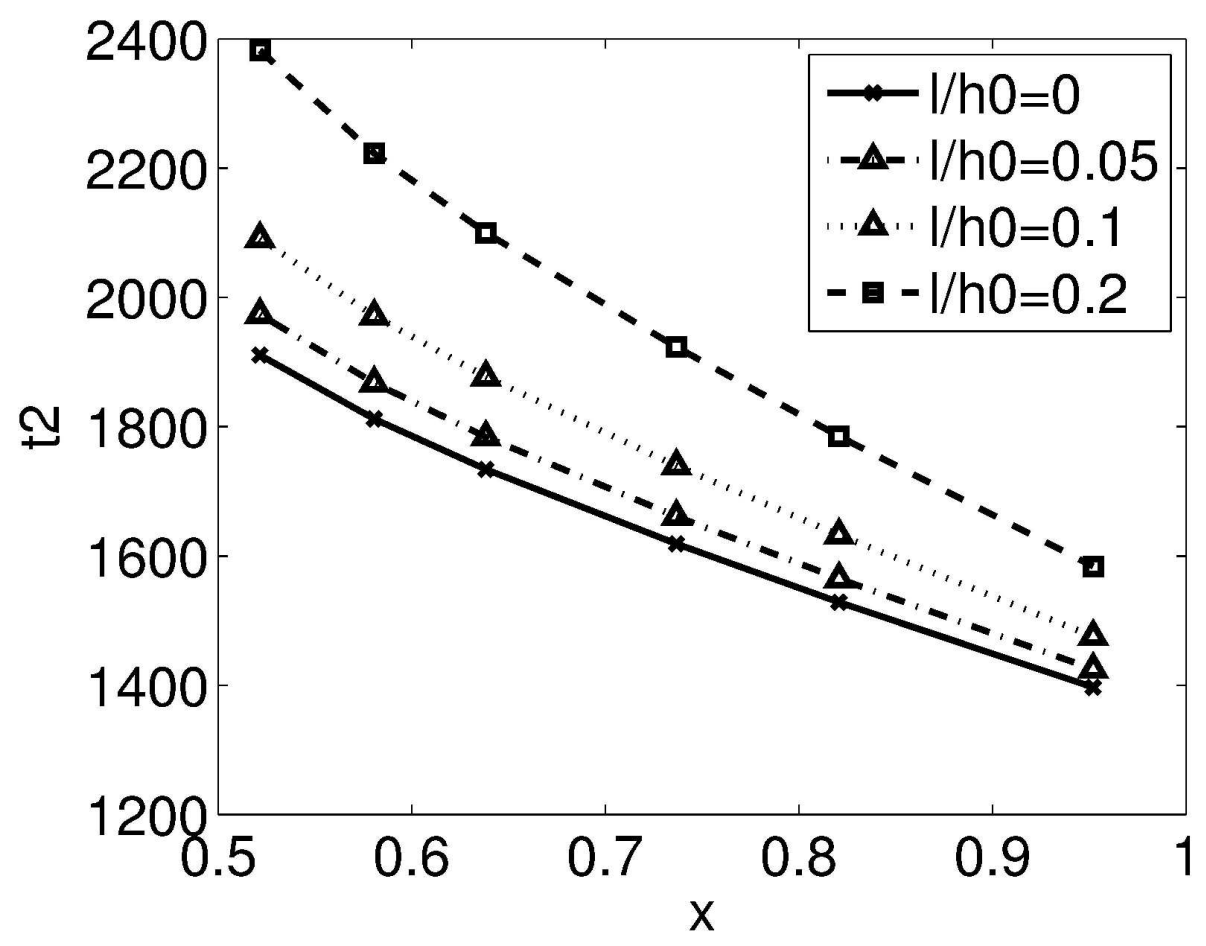

$148 \times 111 \mathrm{~mm}(600 \times 600 \mathrm{DPI})$ 


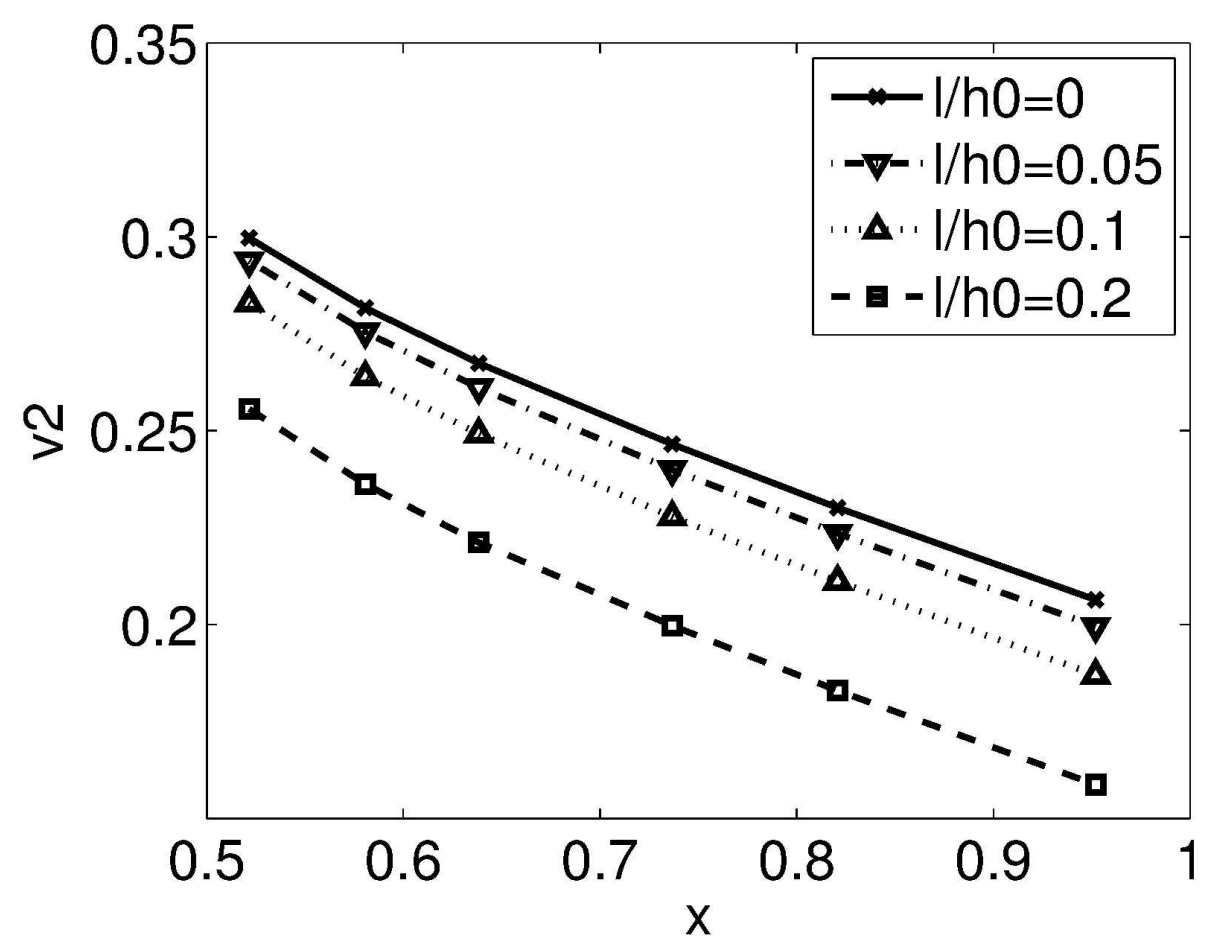

$148 \times 111 \mathrm{~mm}(600 \times 600 \mathrm{DPI})$ 


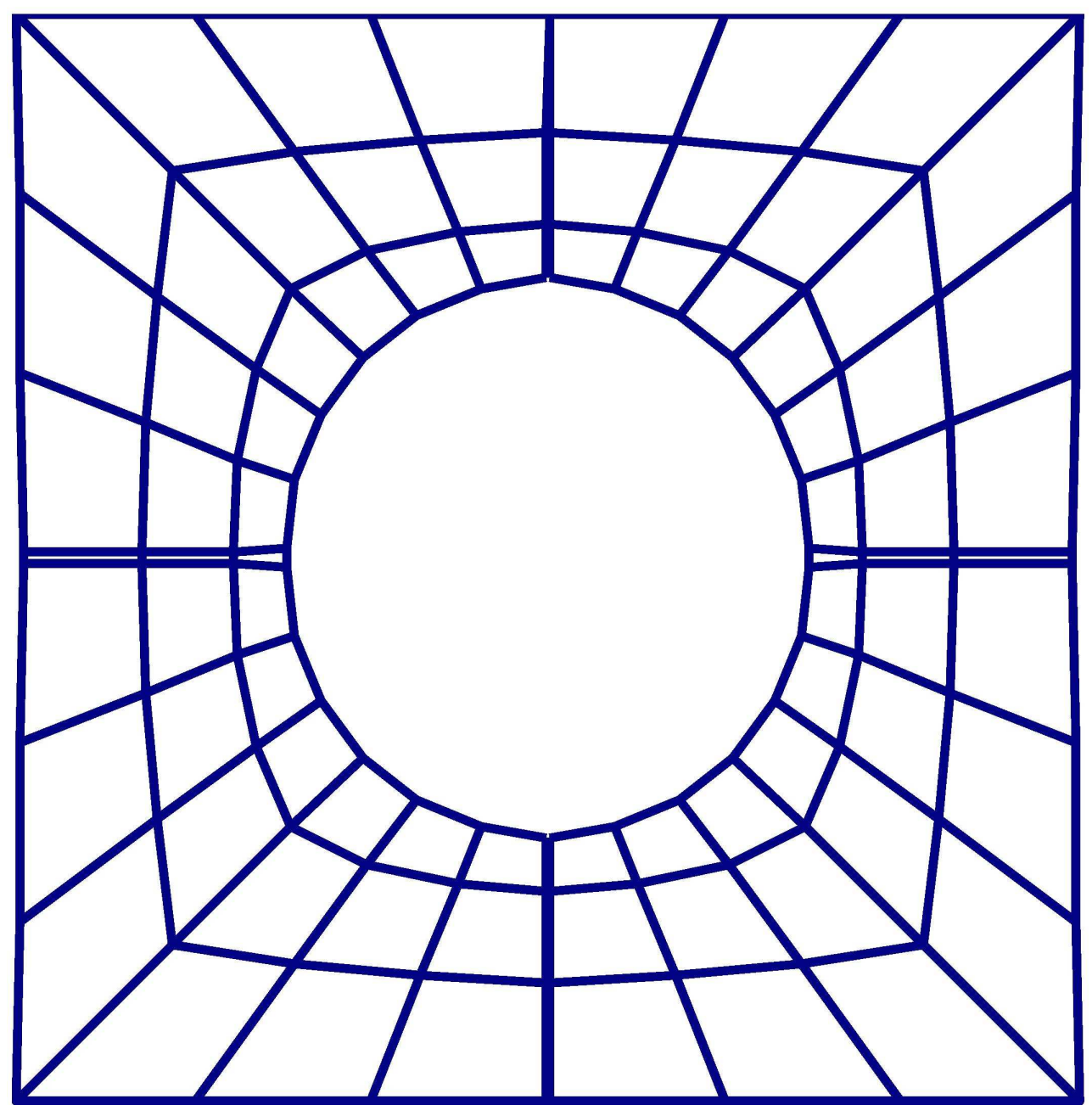

$92 \times 92 \mathrm{~mm}(600 \times 600$ DPI $)$ 


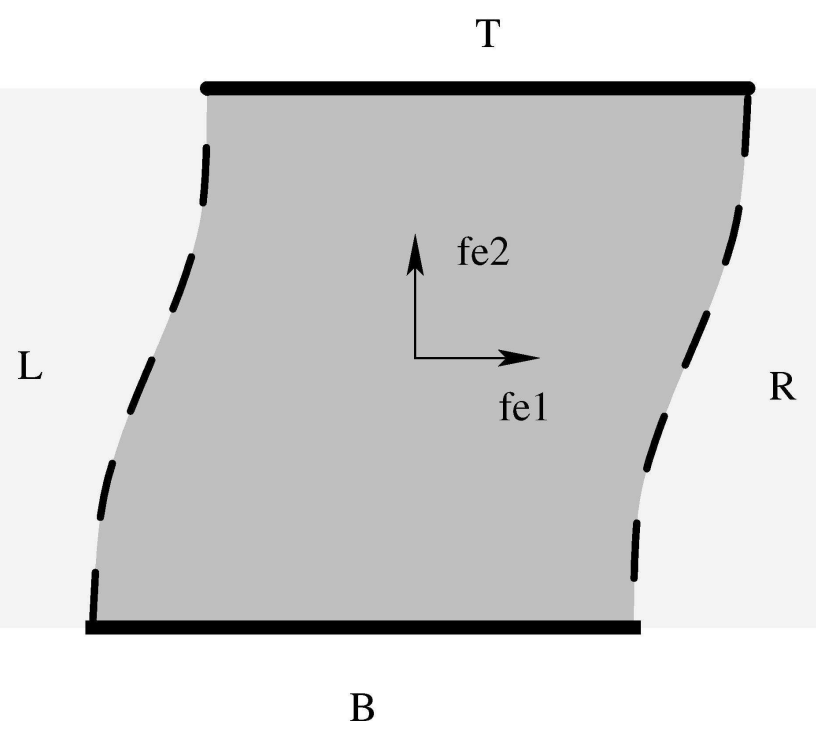

Hybrid boundary conditions on the RVE. $125 \times 68 \mathrm{~mm}(600 \times 600 \mathrm{DPI})$ 


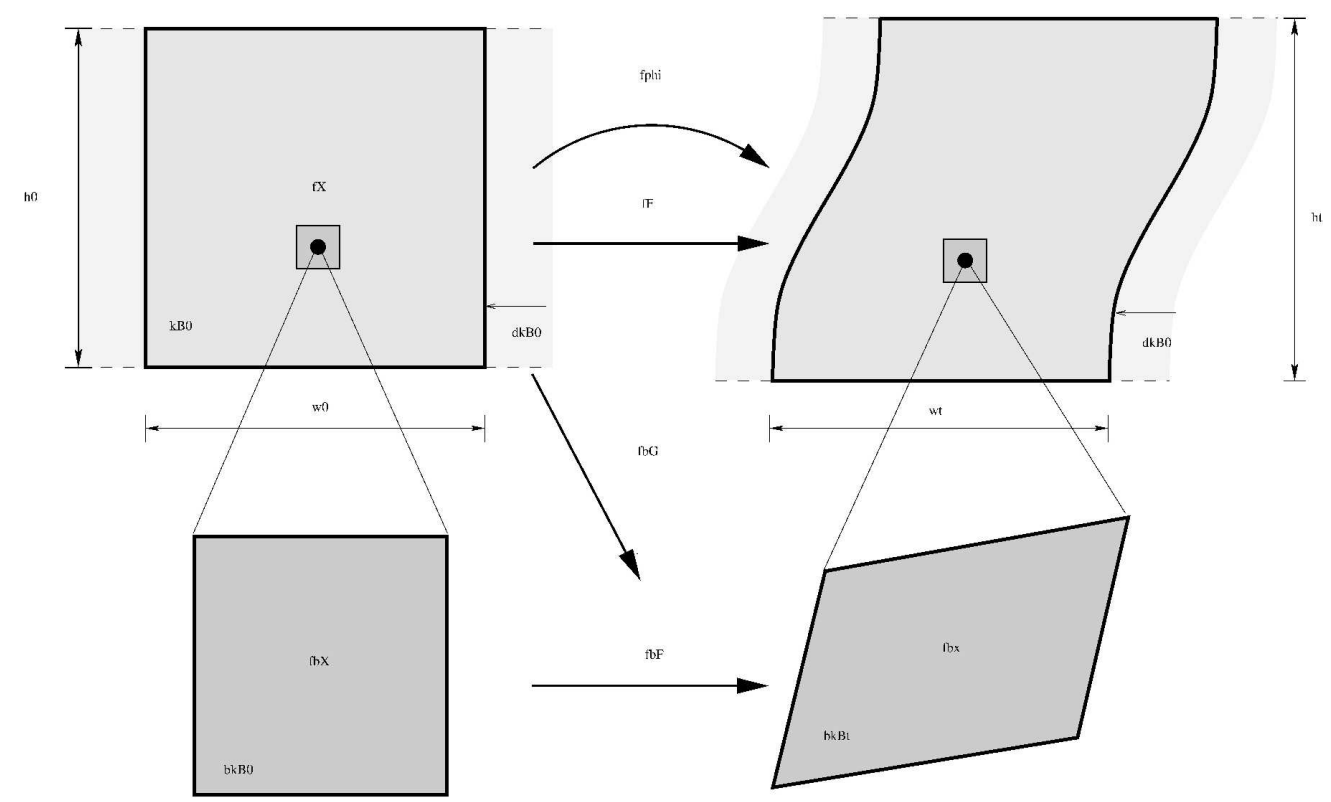

Micromorphic representative volume element: deformation maps. $383 \times 231 \mathrm{~mm}(600 \times 600 \mathrm{DPI})$ 


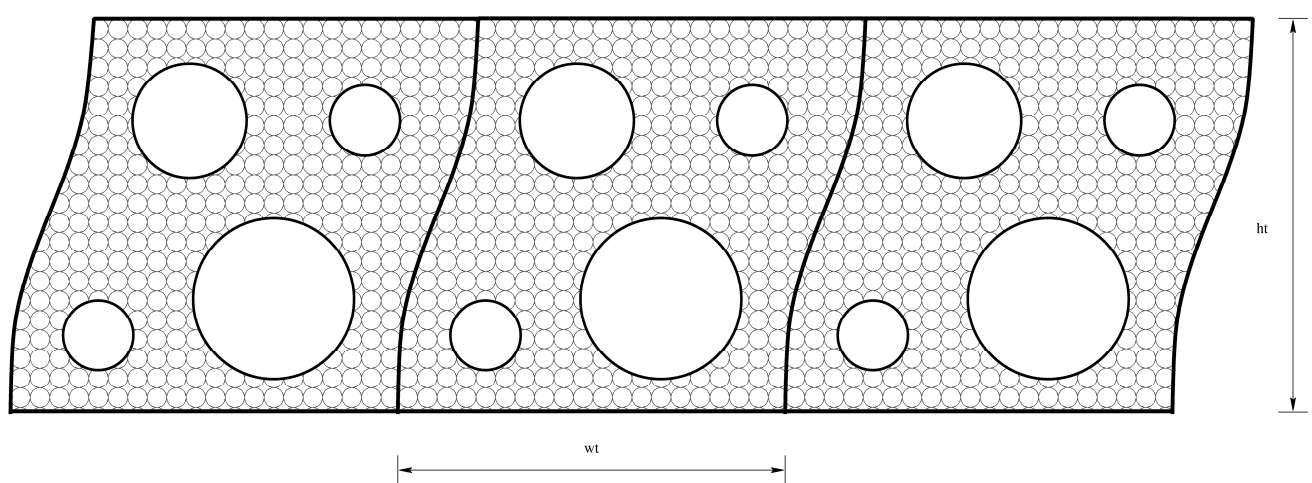

Material layer with periodic mesostructure and inherent microstructure: choice of the RVE height and width

$356 \times 129 \mathrm{~mm}(600 \times 600 \mathrm{DPI})$ 

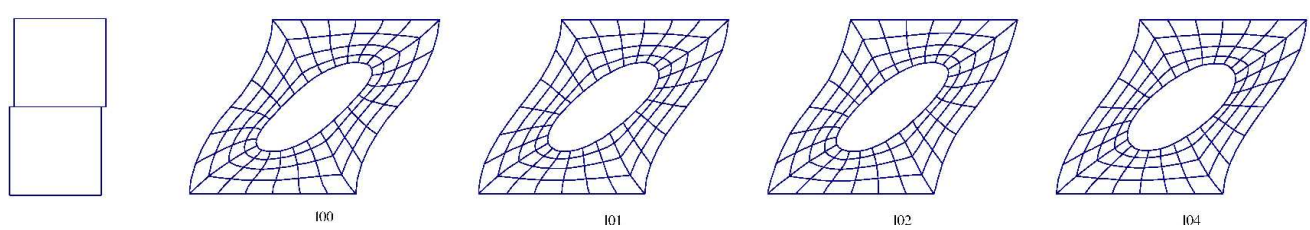

13

14

15

16

17

18

19

20

21

22

23

24

25

26

27

28

29

30

31

32

33

34

35

36

37

38

39

40

41

42

43

44

45

46

47

48

49

50

51

52

53

54

55

56

57

58

59

60

$360 \times 58 \mathrm{~mm}(600 \times 600$ DPI $)$ 

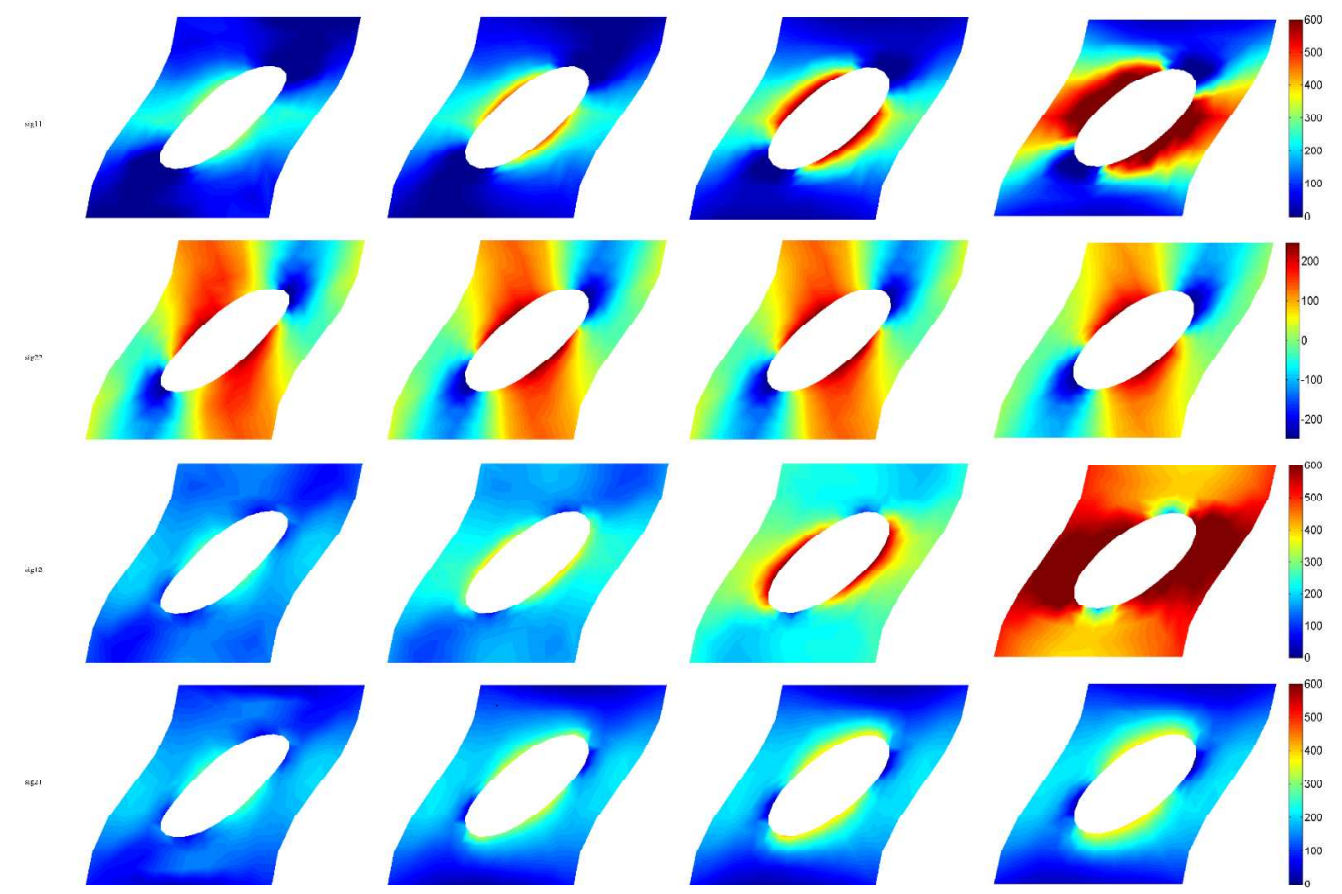

$646 \times 447 \mathrm{~mm}(600 \times 600 \mathrm{DPI})$ 

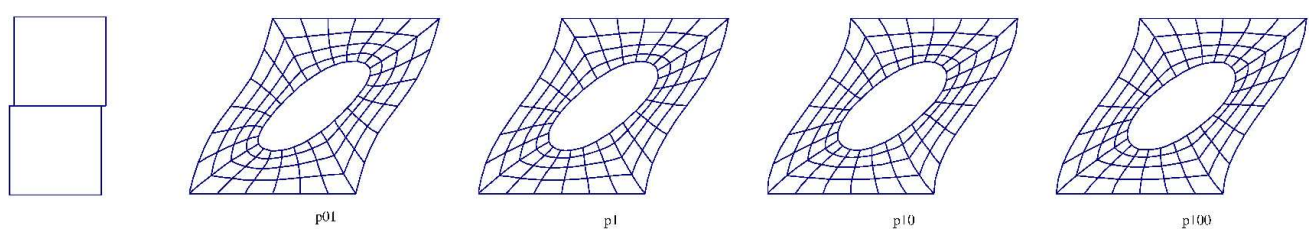

$360 \times 59 \mathrm{~mm}(600 \times 600$ DPI $)$

16

17

18

19

20

21

22

23

24

25

26

27

28

29

30

31

32

33

34

35

36

37

38

39

40

41

42

43

44

45

46

47

48

49

50

51

52

53

54

55

56

57

58

59

60

http://mc.manuscriptcentral.com/pm-pml 

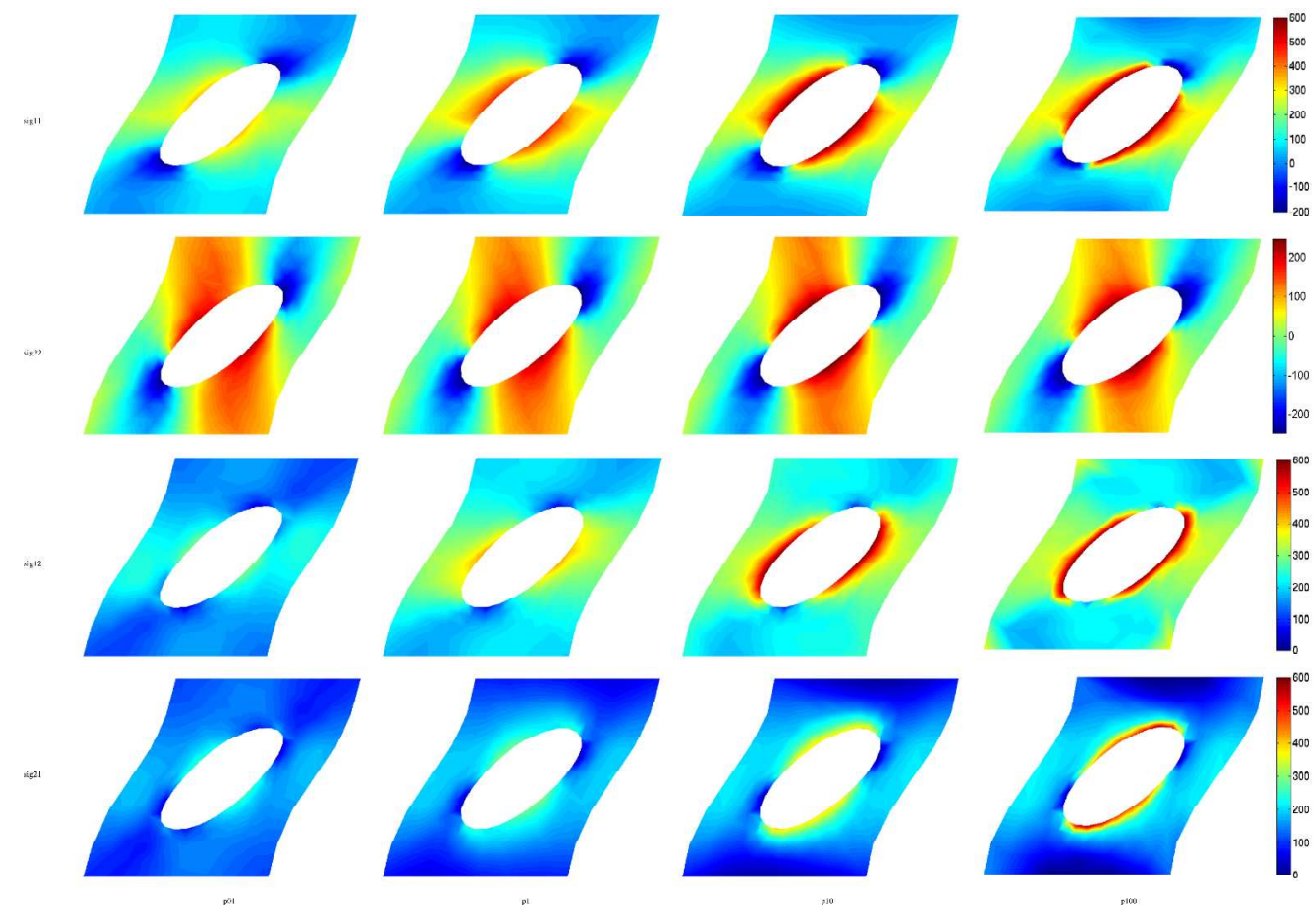

$646 \times 448 \mathrm{~mm}(600 \times 600 \mathrm{DPI})$ 


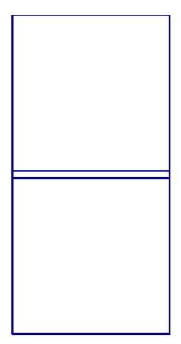

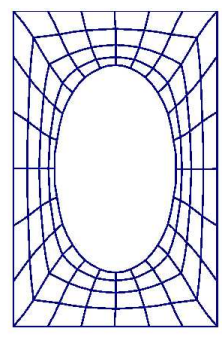

100

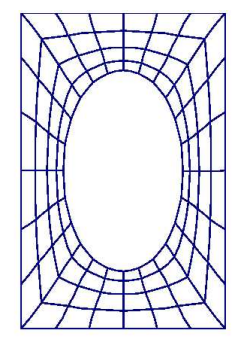

101

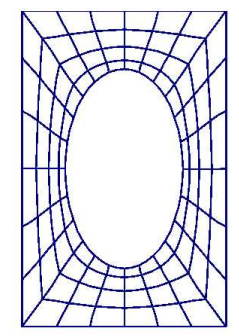

102

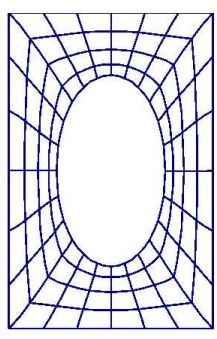

1014

$367 \times 102 \mathrm{~mm}(600 \times 600 \mathrm{DPI})$ 

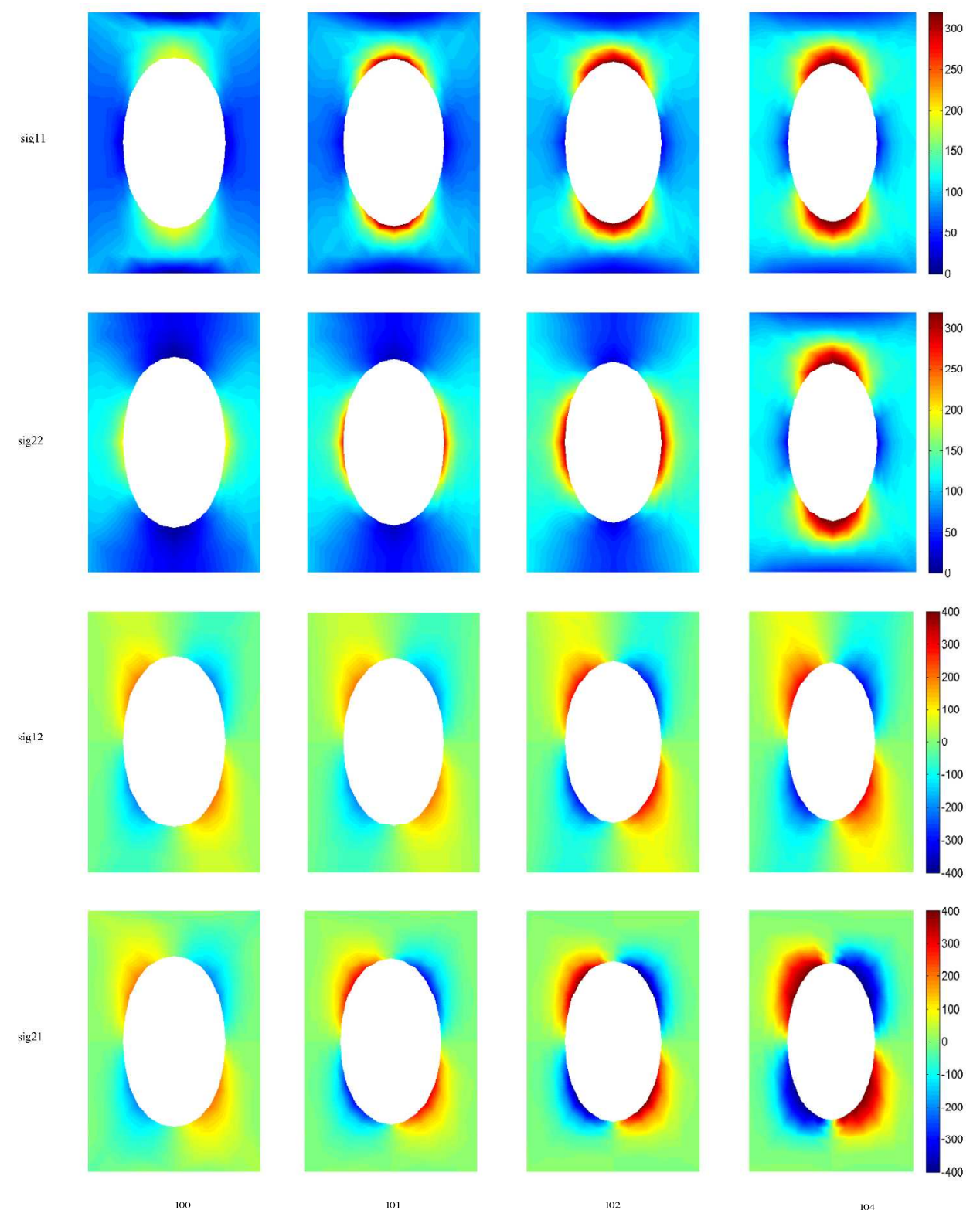
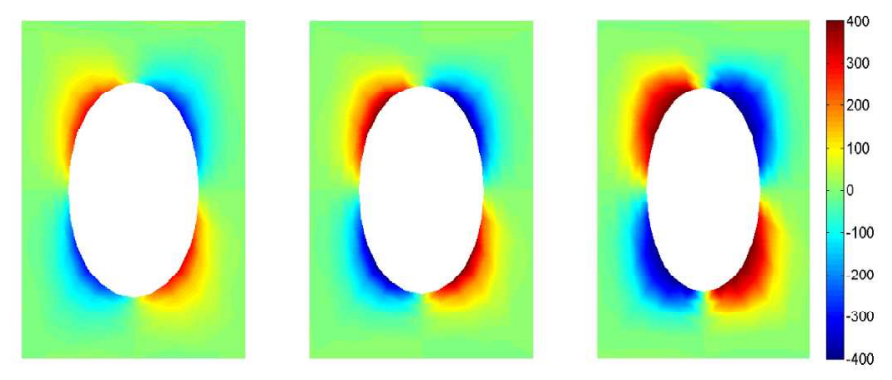

$324 \times 413 \mathrm{~mm}(600 \times 600 \mathrm{DPI})$ 


1
2
3
4
5
6
7
8
9
10
11
12
13
14
15
16
17
18
19
20
21
22
23
24
25
26
27
28
29
30
31
32
33
34
35
36
37
38
39
40
41
42
43
44
45
46
47
48
49
50
51
52
53
54
55
56
57
58
60

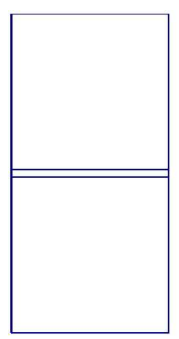

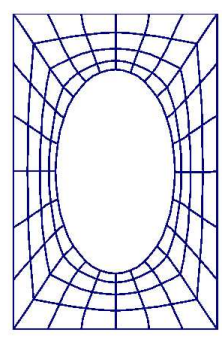

p100

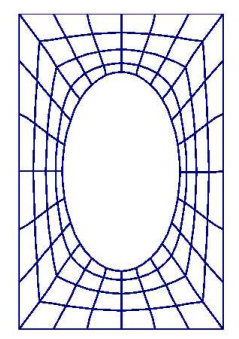

p10

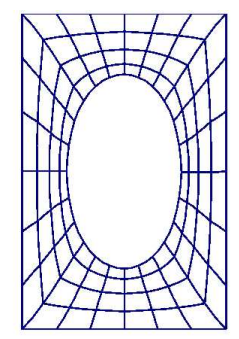

p)

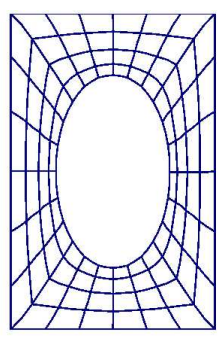

p0I

$367 \times 104 \mathrm{~mm}(600 \times 600$ DPI $)$

http://mc.manuscriptcentral.com/pm-pml 

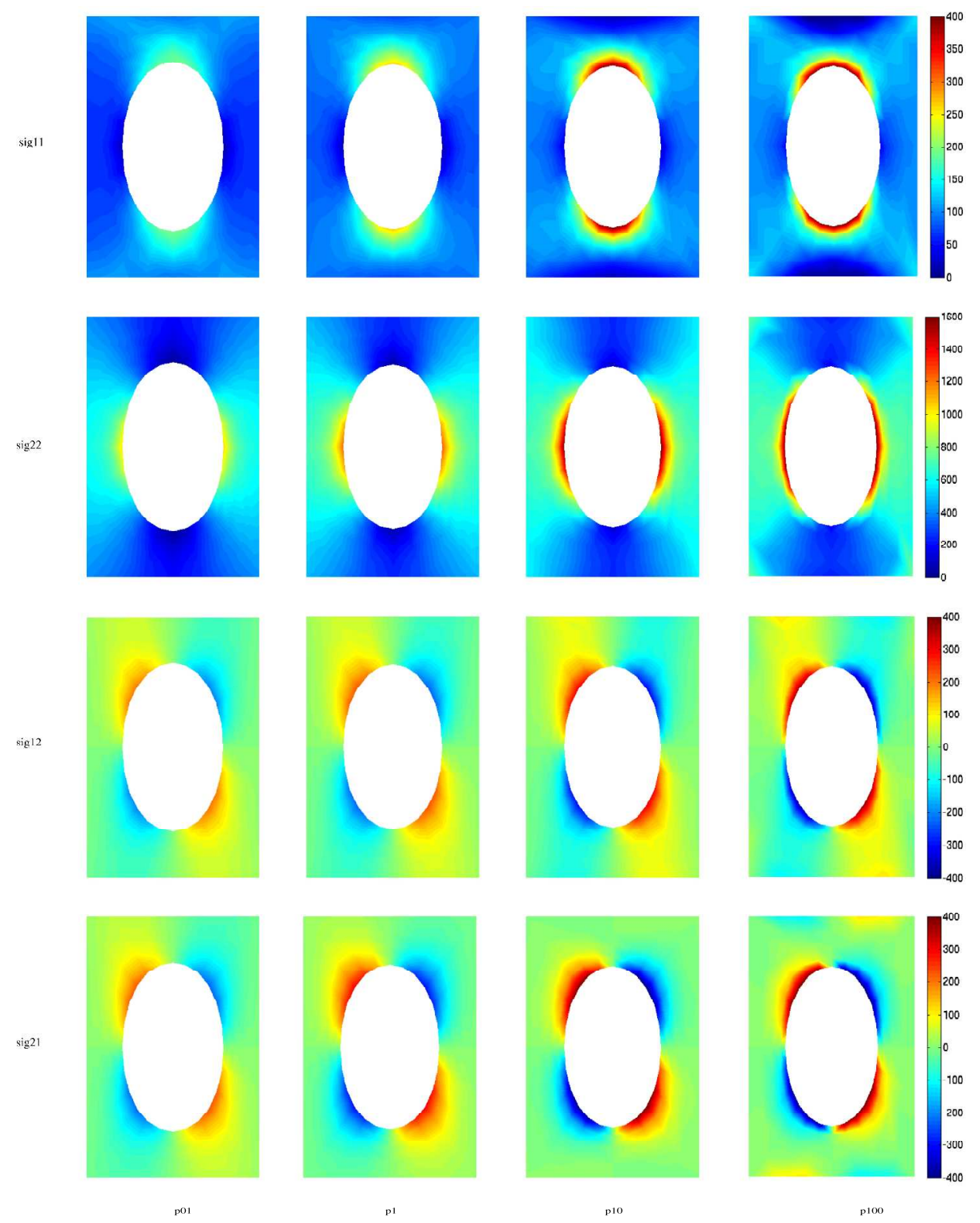

p10

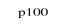

$324 \times 413 \mathrm{~mm}(600 \times 600 \mathrm{DPI})$ 
Computational homogenization between the interface integration point (IP) of the interface element at the macro level and the underlying representative volume element. $291 \times 261 \mathrm{~mm}(600 \times 600 \mathrm{DPI})$ 


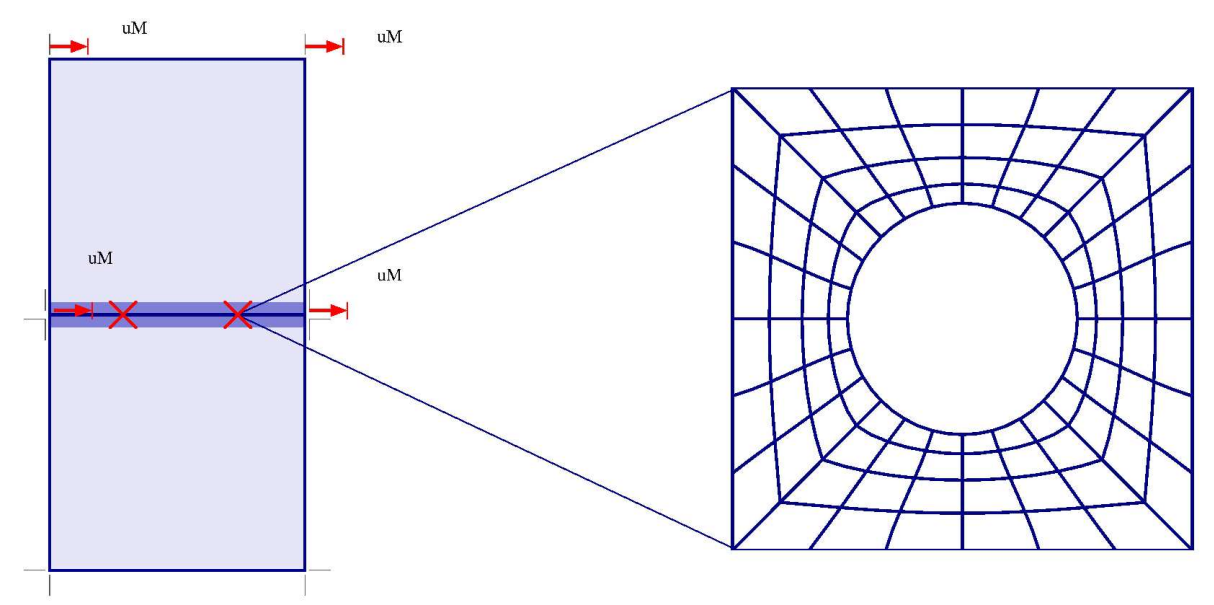

Benchmark problem for multiscale framework: Single interface element subjected to shear. $306 \times 139 \mathrm{~mm}(600 \times 600 \mathrm{DPI})$ 


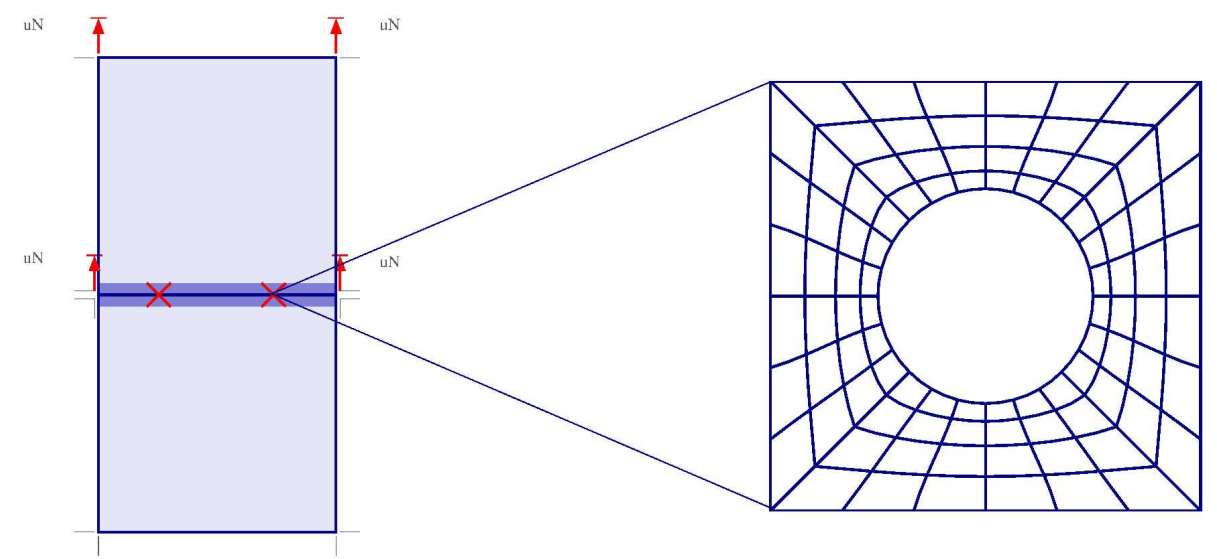

Benchmark problem for multiscale framework: Single interface element subjected to tension. $329 \times 140 \mathrm{~mm}(600 \times 600 \mathrm{DPI})$ 
Example 6.3: Infinite periodic shear layer including material layer: multiscale boundary value problem. $308 \times 145 \mathrm{~mm}(600 \times 600 \mathrm{DPI})$ 
1

2

3

4

5

6

7

8

(1)

10

11

12

13

14

15

16

17

18

19

20

21

22

23

24

25

26

27

28

29

30

31

32

33

34

35

36

37

38

39

40

41

42

43

44

45

46

47

48

49

50

51

52

53

54

55

56

57

58

59

60

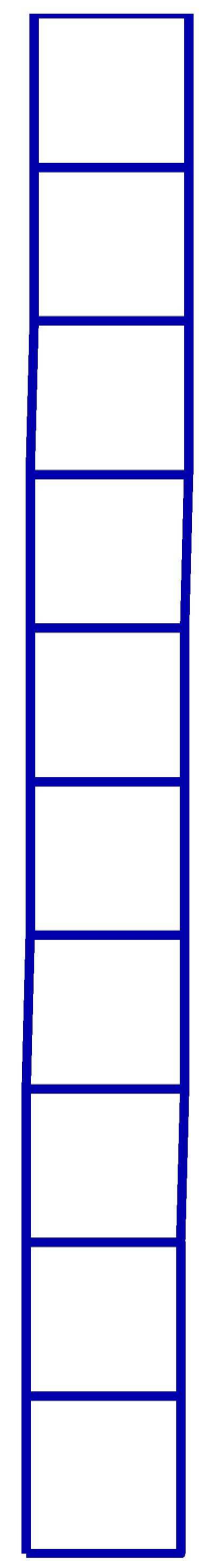

$16 \times 125 \mathrm{~mm}(600 \times 600 \mathrm{DPI})$

http://mc.manuscriptcentral.com/pm-pml 


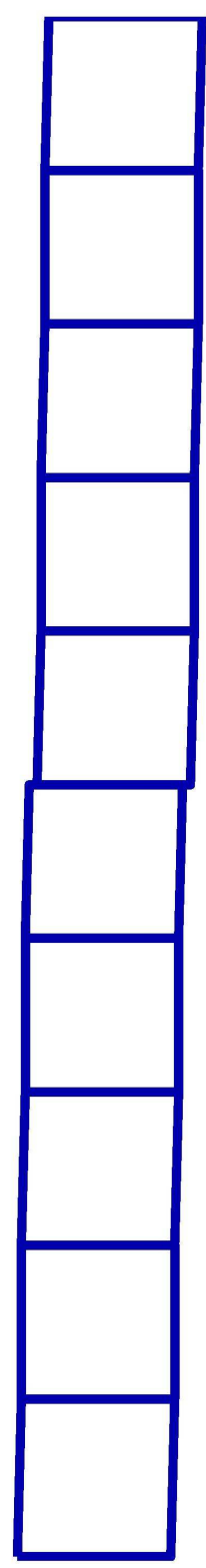

Example 6.3 spatial macro meshes for RVE with (a) hole, (b) vertical lens-shaped void, (c) horizontal lens-shaped void, (d) homogeneous shear layer. $18 \times 125 \mathrm{~mm}(600 \times 600 \mathrm{DPI})$ 


1
2
3
4
5
6
7
8
9
10
11
12
13
14
15
16
17
18
19
20
21
22
23
24
25
26
27
28
29
30
31
32
33
34
35
36
37
38
39
40
41
42
43
44
45
46
47
48
49
50
51
52
53
54
55
56
57
58
59
60

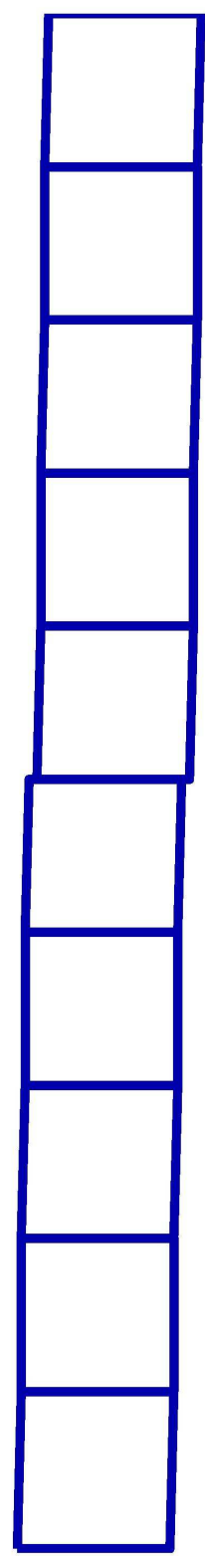

$18 \times 125 \mathrm{~mm}(600 \times 600 \mathrm{DPI})$ 


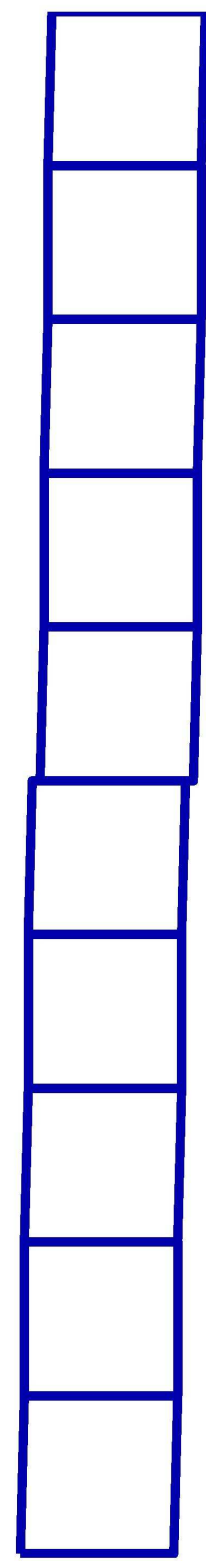

$18 \times 125 \mathrm{~mm}(600 \times 600 \mathrm{DPI})$ 


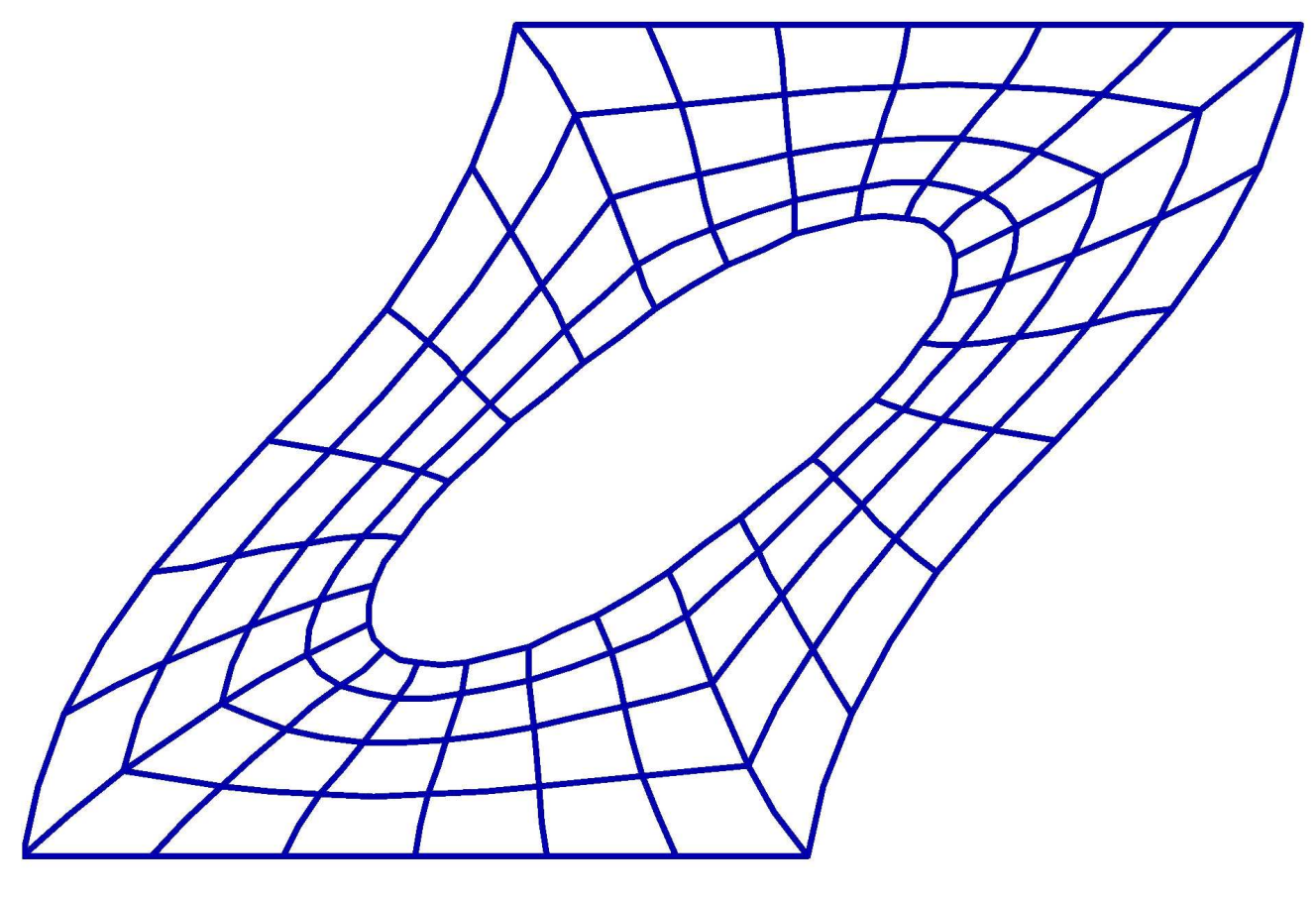

$158 \times 105 \mathrm{~mm}(600 \times 600$ DPI $)$ 


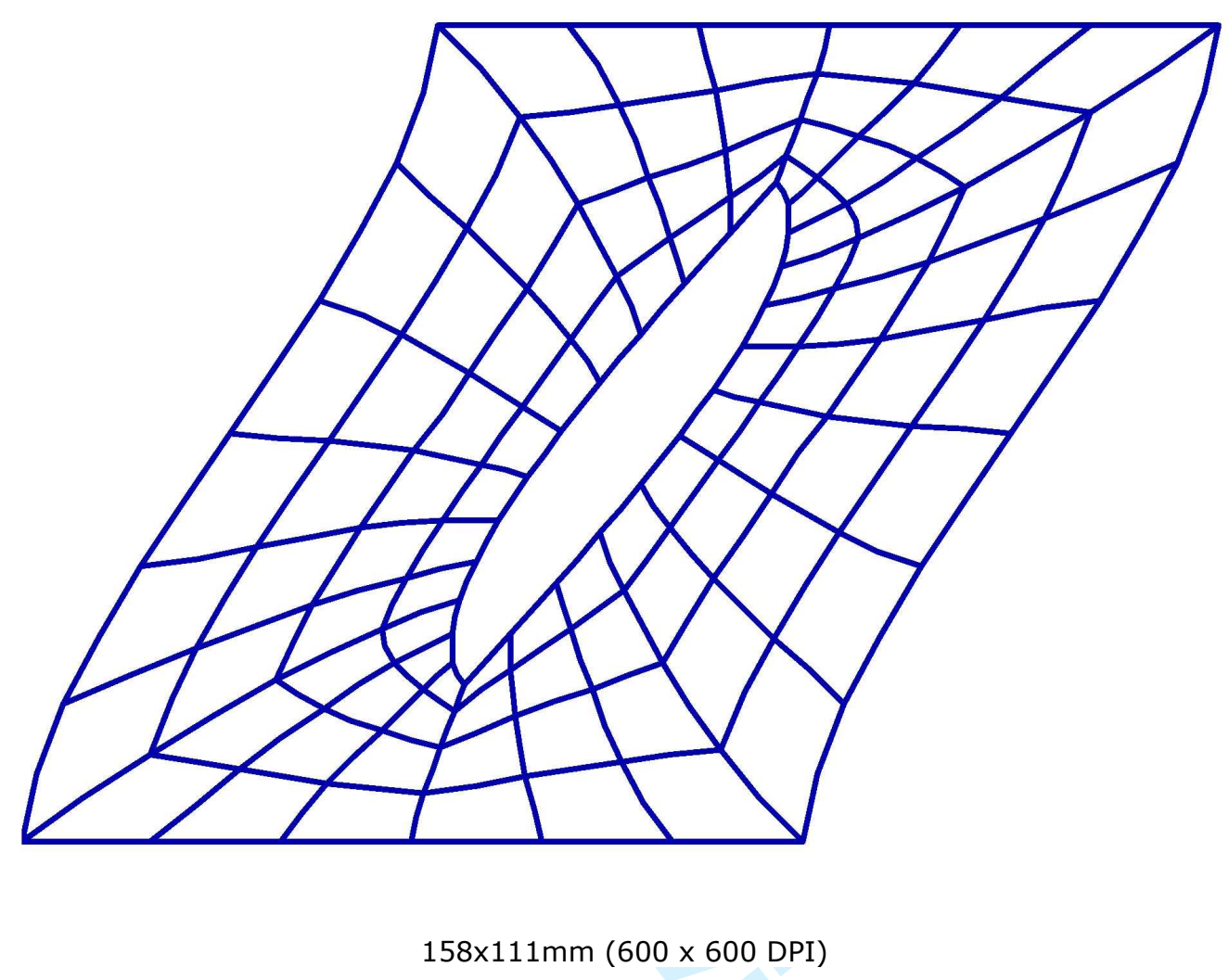




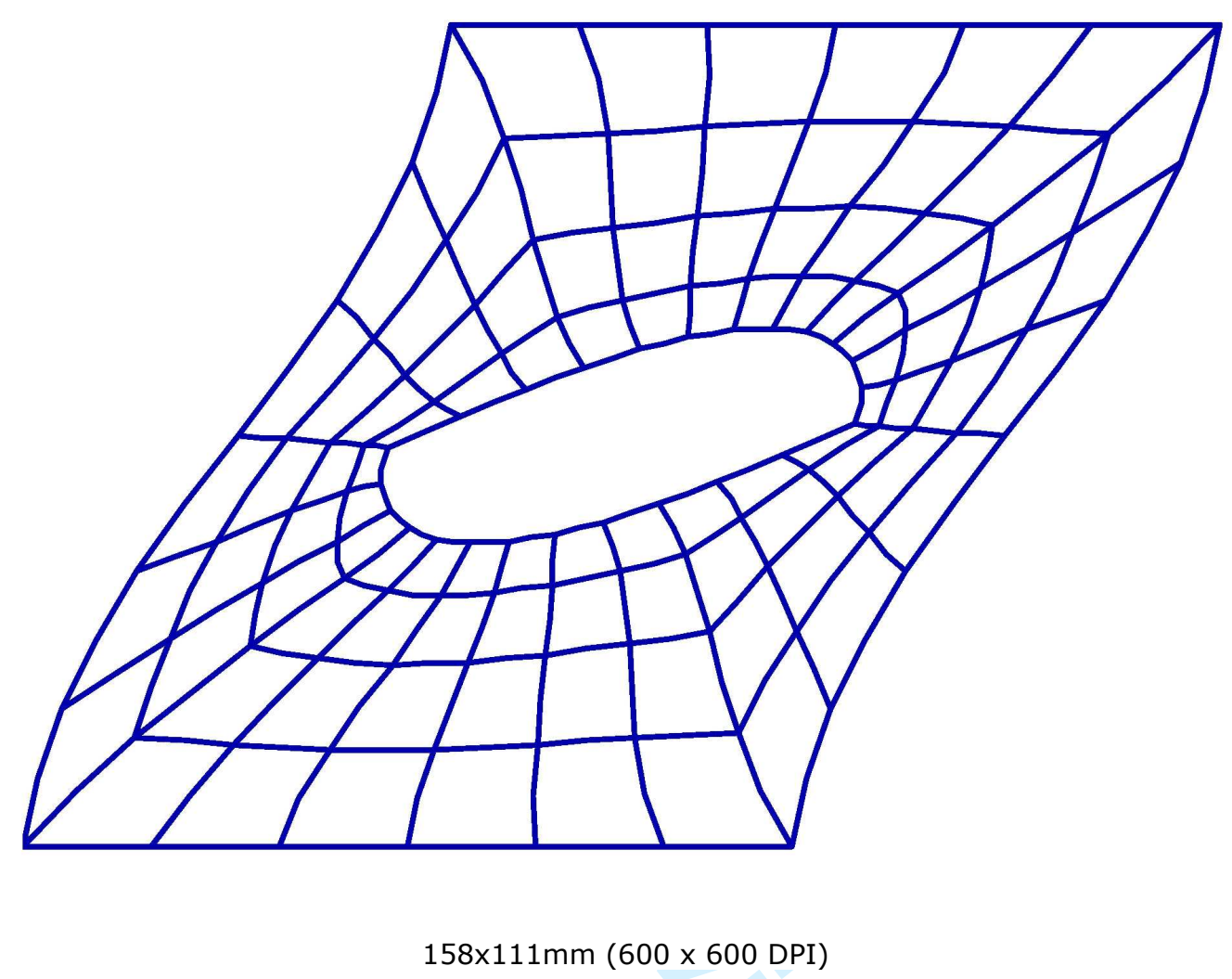




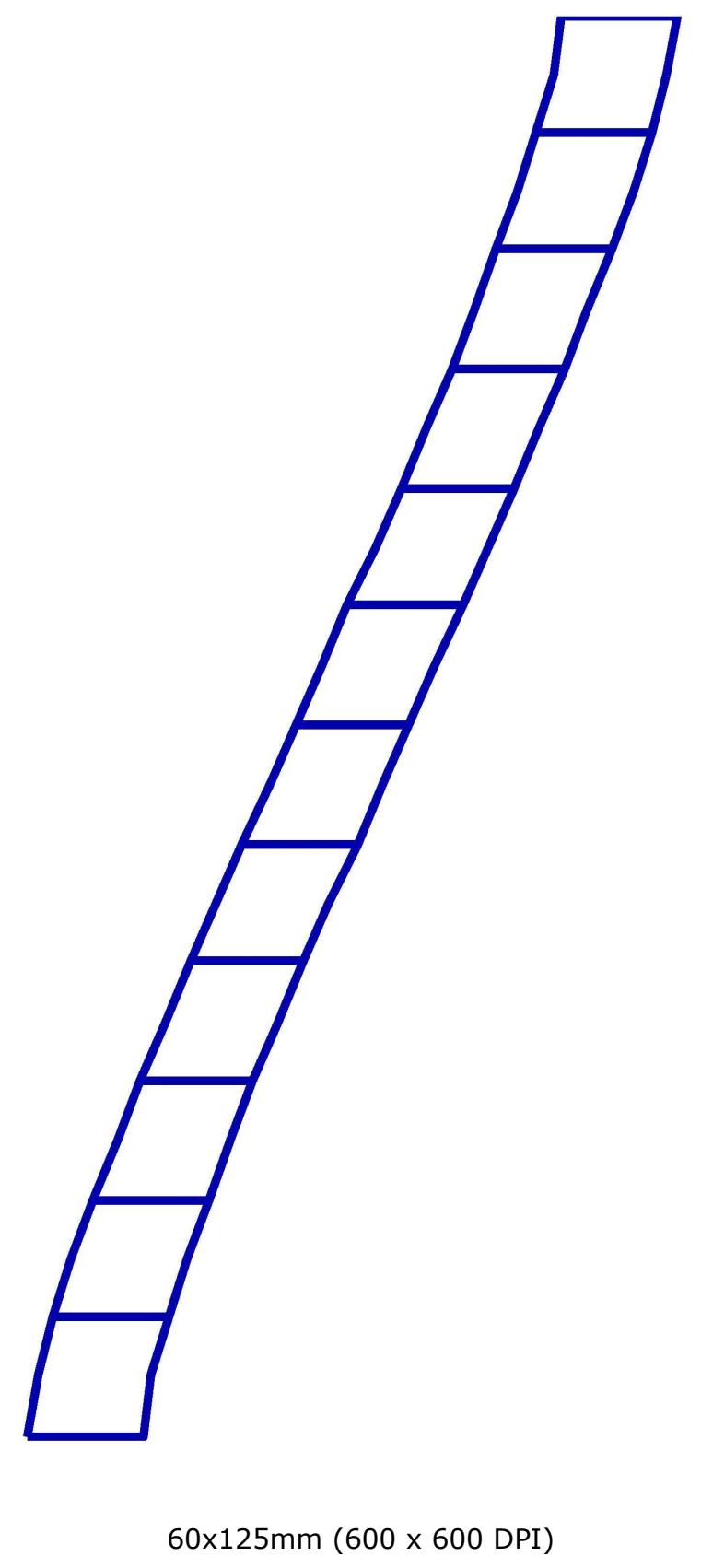

http://mc.manuscriptcentral.com/pm-pml 


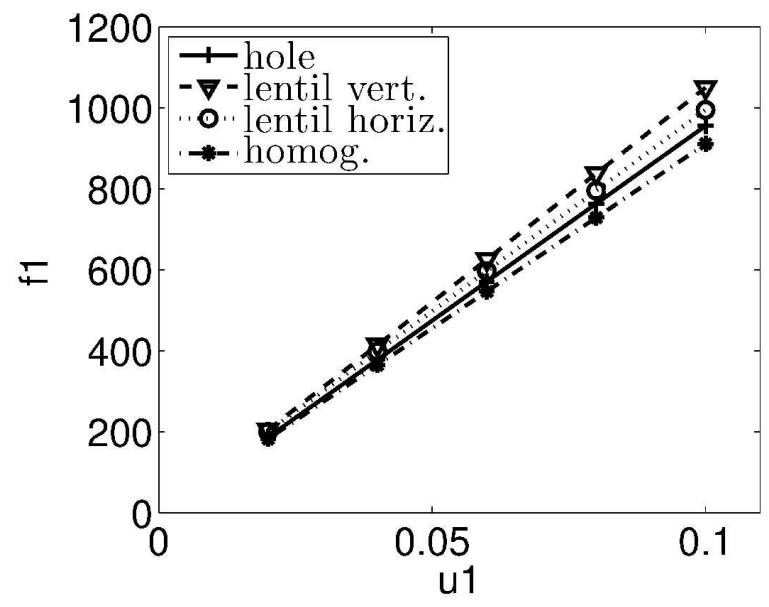

Example 6.3: macro force-displacement curve at top of the macro shear layer $215 \times 279 \mathrm{~mm}(600 \times 600 \mathrm{DPI})$ 


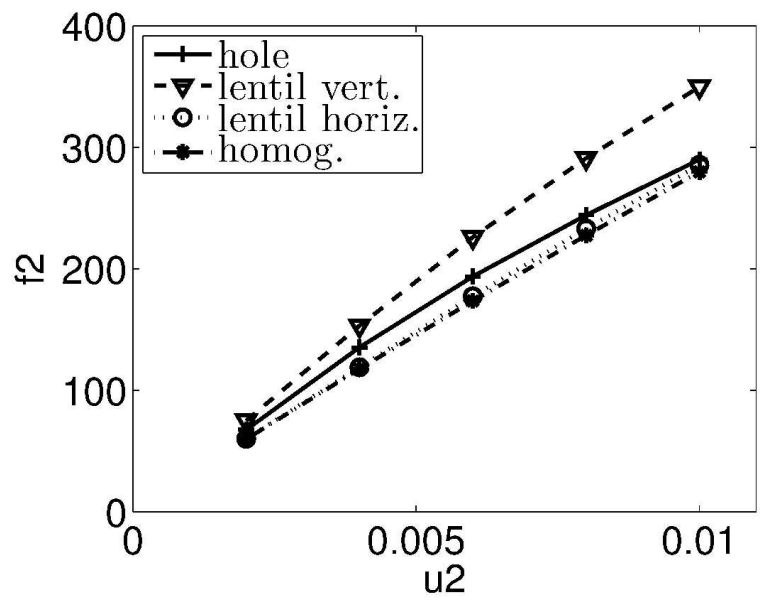

$215 \times 279 \mathrm{~mm}(600 \times 600 \mathrm{DPI})$

http://mc.manuscriptcentral.com/pm-pml 


1
2
3
4
5
6
7
8
9
10
11
12
13
14
15
16
17
18
19
20
21
22
23
24
25
26
27
28
29
30
31
32
33
34
35
36
37
38
39
40
41
42
43
44
45
46
47
48
49
50
51
52
53
54
55
56
57
58
60

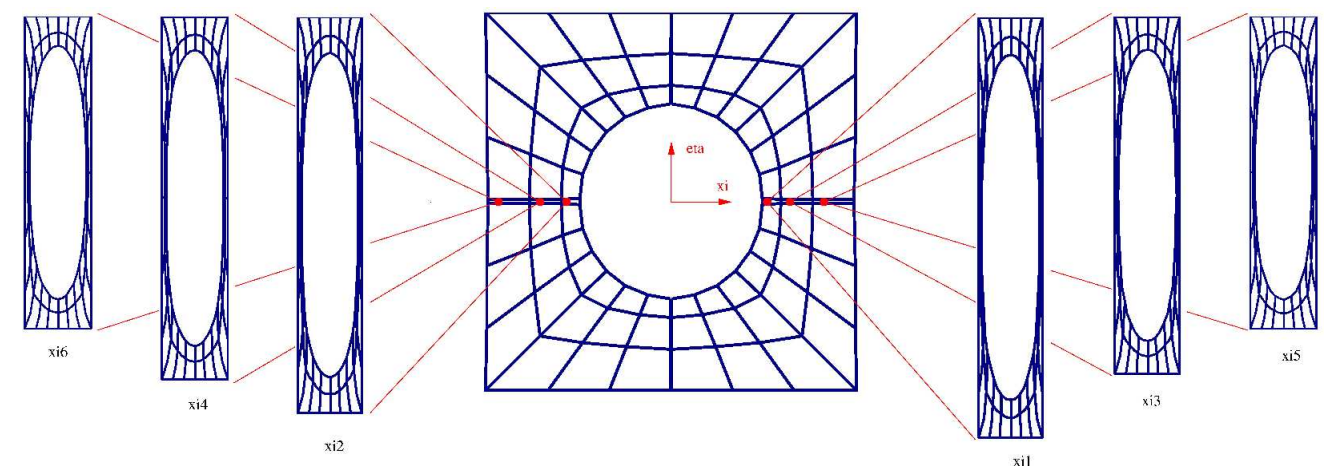

$344 \times 121 \mathrm{~mm}(600 \times 600$ DPI $)$ 